12

\title{
Перспективные технологии для прикладных резонансных ускорителей заряженных частиц и примеры их использования (Обзор)
}

\author{
(C) C.B. Куцаев \\ RadiaBeam Technologies LLC, \\ Санта Моника, Калифорния, США \\ e-mail: kutsaev@radiabeam.com
}

Поступило в Редакцию 13 июня 2020 г.

В окончательной редакции 1 сентября 2020 г.

Принято к публикации 7 сентября 2020 г.

В обзоре представлено видение автором современных тенденций развития ускорителей заряженных частиц для прикладного применения, составленное на основе собственного опыта работы в данной сфере. Показаны наиболее, по мнению автора, перспективные области применения резонансных ускорителей, в которых достигнут значительный прогресс благодаря применению новых технологий. Использование структур с высокими градиентами, новых материалов и технологий изготовления, систем охлаждения и новых принципов ускорения позволило добиться миниатюризации, повышения экономической эффективности и возможности изменения параметров (вариативности) ускорителей для промышленного, медицинского и научного применения, а также для систем безопасности и квантовых компьютеров.

Ключевые слова: линейные ускорители, замена радиоизотопов, радиотерапия, инспекция грузов, ядерная безопасность, электронно-лучевая обработка, сверхпроводящие ускорители.

DOI: 10.21883/JTF.2021.02.50352.201-20

\section{Введение}

Пучки заряженных частиц (электронов и ионов), нейтронов и фотонов, полученные с помощью ускорителей заряженных частиц, широко применяются для решения прикладных задач. За последнее десятилетие в некоторых областях их применения, таких как инспекция грузов [1], ядерная безопасность [2], радиотерапия для лечения опухолей $[3,4]$, промышленное облучение материалов [5-7], пищевых и сельскохозяйственных продуктов, медицинских изделий $[8,9]$ и др., наблюдается значительный прогресс, который стал возможным благодаря появлению новых технологий для ускорителей. Многие из этих задач ставят новые требования к источникам излучения, ускорителям заряженных частиц. К этим требованиям относятся компактность, мобильность (от возможности передислокации до переноса в руках), минимизация потребления энергии и автономность, быстрая перестройка параметров пучка, а также снижение стоимость постройки ускорителя и его обслуживания.

В настоящей работе будут разобраны примеры современных и перспективных прикладных задач, которые невозможно реализовать в полной мере с помощью существующих коммерческих ускорителей. Для каждой задачи будут сформулированы требования к источникам, а также описаны новые ускорительные технологии и методы, позволяющие их реализовать.

\section{1. Требования к современным источникам и тенденции их разработок}

Большинство тенденций определяются следующими требованиями [10]:

- миниатюризация: уменьшение размеров ускорителей и создание более компактных установок;

- вариативность параметров пучка как для конкретной задачи, так и для реализации многозадачности;

- повышение экономической эффективности, которая может быть реализована как снижением эксплуатационных, производственных затрат, так и повышением энергетической эффективности и т.д.

В следующих секциях будут подробно рассмотрены причины и способы реализации указанных тенденций. Данный обзор ограничен рассмотрением работ по применению современных резонансных ускорителей и не затрагивает вопросы использования для этих целей малогабаритных ускорителей прямого действия и плазменных ускорителей, которые также могут быть перспективны с точки зрения использования в переносных и бортовых устройствах дистанционного ядерного контроля [11].

\section{1. Миниатюризация}

Уменьшение размера и веса ускорителя имеет огромное значение для таких систем, как инспекция грузов. 
Например, в случае патрулирования границ, ускоритель вместе с системой питания, защитой, системой контроля и детекторной станцией должен помещаться на автомобиль для быстрой смены дислокации. Ускорители для медицинского применения должны также помещаться на мобильную платформу, чтобы обеспечить доступ пучка со всех сторон опухоли, что особенно важно для таких современных технологий, как 4л-радиотерапия [12] или FLASH-терапия [13].

Промышленное применение переносных ускорителей включает в себя облучение крови [14], выездную радиографию [15], каротаж нефтяных скважин [16], а также системы безопасности, в которых в настоящее время используются радиоизотопы [17]. Устройства на основе радиоизотопных источников излучения имеют тенденцию к замене на безопасные технологии, такие, как ускорители заряженных частиц (из-за риска использования радиоактивных материалов террористами в качестве оружия [18]). В случае такой замены ускорители должны иметь сравнимые с радиоизотопными источниками размеры.

Наибольший вклад в размеры и вес традиционных ускорителей вносят источники питания и радиационной защиты. Габариты слоя защиты напрямую зависят от размера ускоряющей секции. Одним из путей уменьшения размеров данных компонентов является повышение частоты колебаний электромагнитного поля в ускорителях. Более высокочастотные структуры и источники питания имеют меньшие размеры, а также энергетически эффективны. Например, такой важнейший критерий эффективности преобразования энергии ВЧ-поля в энергию пучка, как шунтовое сопротивление, определяемый как отношение квадрата прироста энергии частицы к рассеянной в структуре мощности $\left(R_{s h}=V^{2} / P\right)$, увеличивается с частотой как $f^{1 / 2}$ для традиционных структур [19]. В случае же структур на стоячей волне запасенная энергия, определяемая добротностью резонатора $Q$, уменьшается пропорционально $f^{-1 / 2}$, что уменьшает интервал заполнения ВЧ-структуры, работающей в режиме стоячей волны, во время которого мощность не используется для ускорения пучка $\tau=\frac{Q_{L}}{\pi f} \sim f^{-3 / 2}[20]$, что также приводит к повышению эффективности.

Выбор оптимальной частоты зависит от требуемых параметров ускорителя и ВЧ-источника. Традиционные промышленные ускорители работают в $S$-частотном диапазоне $(3 \mathrm{GHz})$ и используют клистрон в качестве источника питания [21]. Клистрон, как усилитель ВЧ-мощности, обладает такими преимуществами, как доступность большой пиковой и средней мощности и фазовая стабильность, однако требует источника задающего напряжения и фокусирующего соленоида, что значительно увеличивает размеры и вес системы ВЧ-питания [22]. В современных ускорителях имеет место тенденция использования более компактных источников ВЧ-энергии - магнетронов. Еще одной альтернативой тради- ционным клистронам является российская разработка многолучевых клистронов $S$-диапазона с реверсной фокусировкой постоянными магнитами, сопоставимых по размерам и характеристикам питания с магнетронами, но имеющие большую среднюю и импульсную мощность и сохраняющие все преимущества клистронов. В последние годы в России был разработан аналогичный клистрон $C$-диапазона с импульсной мощностью $3.5 \mathrm{MW}$. Такие клистроны используются в современных компактных ускорителях электронов [23].

Переход в $C$-частотный диапазон $(6 \mathrm{GHz})$ позволяет иметь мощности, сравнимые с $S$-диапазоном при большей компактности $[24,25]$. Такие ускорители могут получать пучки электронов энергией 9 и более $\mathrm{MeV}$. $X$-частотный диапазон $(9-11 \mathrm{GHz})$ подходит для ускорителей на 4-6 MeV, в то время как Ku-частотный диапазон $(16 \mathrm{GHz})$ позволяет достичь минимальных размеров для ускорителей с энергией порядка $1 \mathrm{MeV}$ [26], что обусловлено мощностью доступных магнетронов. Существуют магнетроны, работающие и на более высоких частотах (35 GHz), например CPI VMA 1647G [27], однако их размеры отличаются от работающих в Ки-диапазоне уже незначительно (рис. 1).

Интересно отметить тенденцию использования ускоряющих структур миллиметрового диапазона $(100 \mathrm{GHz}$ и выше). Основное преимущество таких структур заключается в возможности получения ускоряющих градиентов $>200 \mathrm{MeV} / \mathrm{m}$ [28]. Однако отсутствие компактных источников большой мощности $(>100 \mathrm{~kW})$ на эти частоты является препятствием для их практического использования. На сегодняшний день только громоздкие гиротроны могут произвести необходимую мощность, но даже в этом случае временные параметры выходного импульса (большая длительность и малая частота повторения) не позволяют использовать их в качестве источника питания для ускорителей [29]. Тем не менее быстрое развитие THz-технологий может значительно улучшить перспективы использования таких ускорителей в будущем десятилетии [30-33].

Практическое развитие высокоградиентных структур также имеет два направления: повышение градиентов в структурах сантиметрового диапазона (в основном $X$-частотного диапазона) [34] и использование микроструктур, возбуждаемых лазером или первичным пучком, так называемое кильватерное ускорение $[35,36]$. В первом случае можно выделить современные работы по определению новых критериев ВЧ-пробоя [37], повышению электрической прочности медных структур [38], использование новых сплавов [39] и работы в низкотемпературных $(20-80 \mathrm{~K})$ режимах [40]. В настоящее время удалось достичь градиентов более $250 \mathrm{MV} / \mathrm{m}$ в структурах $X$-частотного диапазона [41], охлажденных до криогенных температур, с перспективой достижения до $500 \mathrm{MV} / \mathrm{m}$ в диэлектрических структурах в будущем [42].

Среди новых методов ускорения, которые разрабатываются в мире, можно отметить три метода кильва- 
$a$
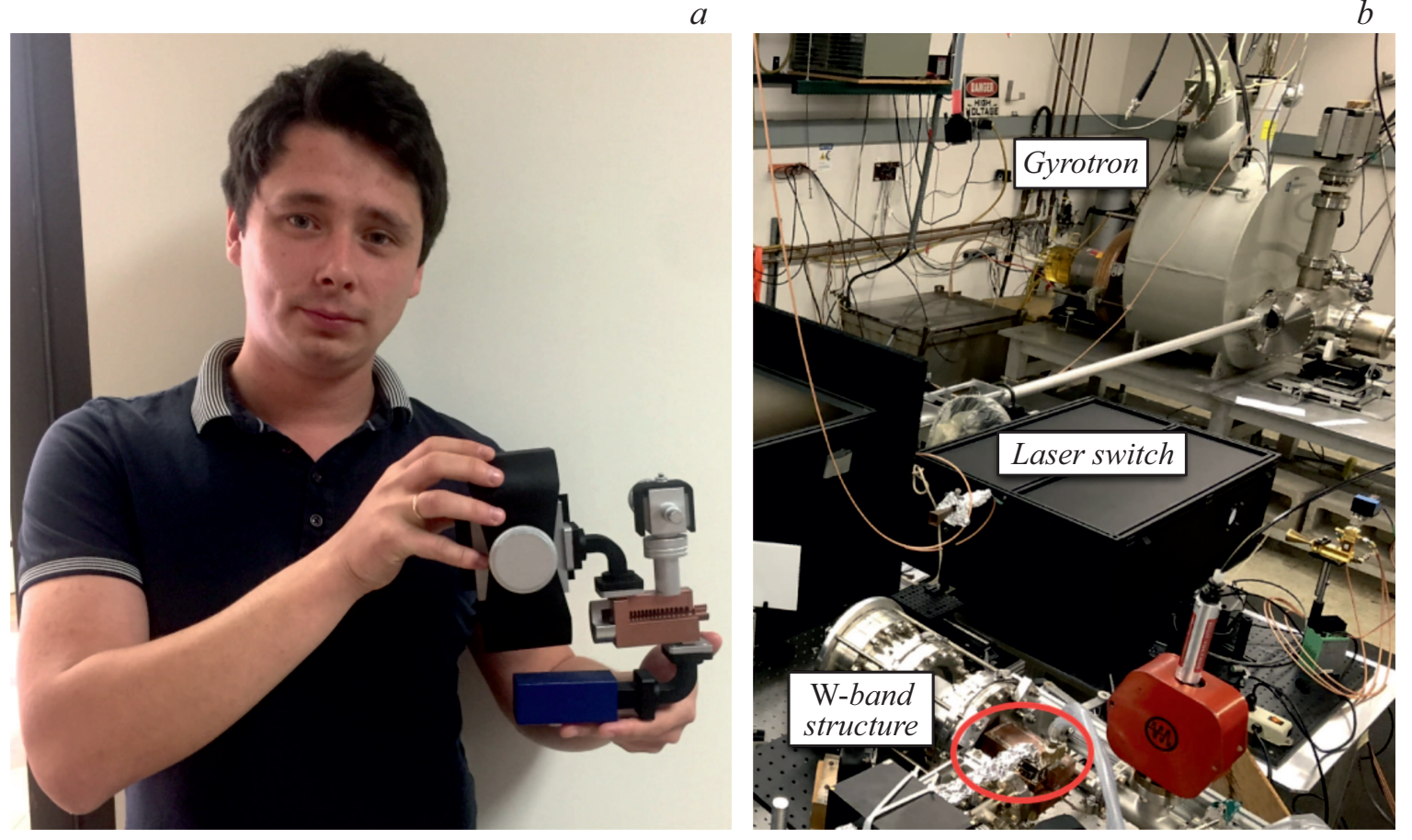

Рис. 1. Ускоряющие структуры $K u$ - $(16.7 \mathrm{GHz})(a)$ и $W$ - $(110 \mathrm{GHz})(b)$ частотных диапазонов вместе с источниками питания. Рисунок хорошо демонстрирует насколько важно учитывать периферические компоненты в оценке размера ускорителя.

$a$
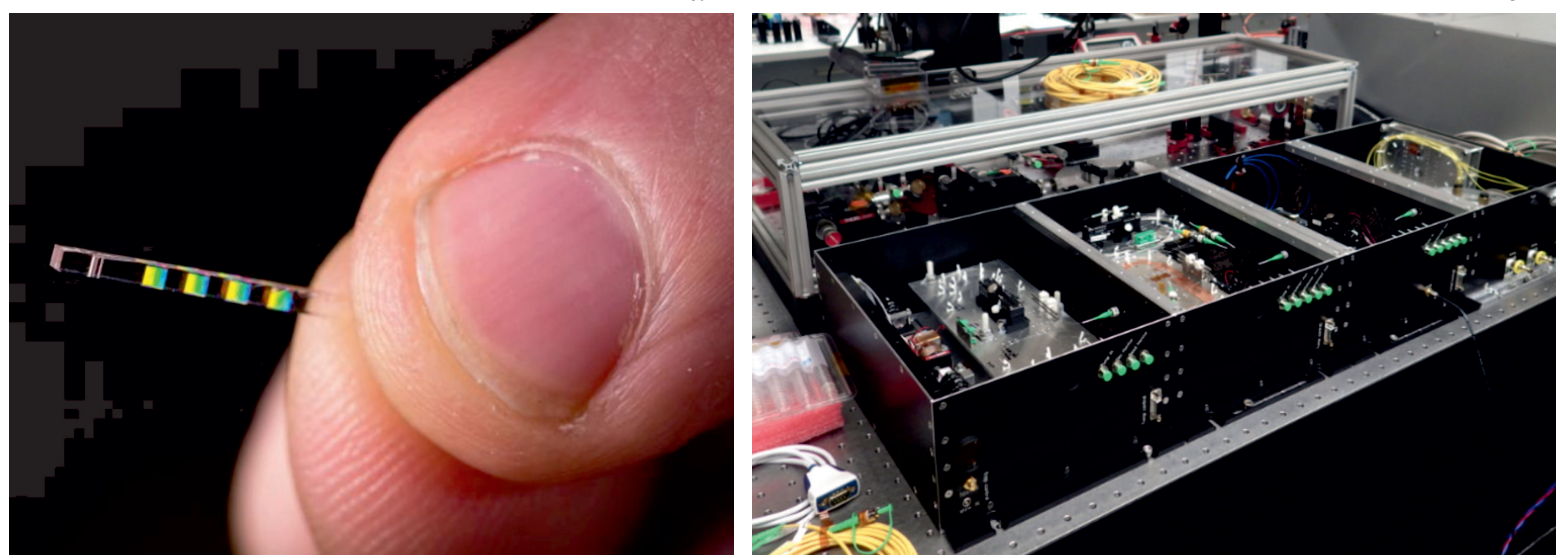

Рис. 2. Один из ранних прототипов диэлектрической ускоряющей структуры для „ускорителя на чипе $(a)$ и современный фемтосекундный лазер для ее возбуждения $(b)$, разработанные в Стэнфордской Национальной Ускорительной Лаборатории SLAC, США [355-357]. Источник фотографий: Accelerator on a Chip International Program (https:/achip.stanford.edu).

терного ускорения, отмеченных в отчете Министерства Энергетики США по стратегии развития перспективных ускорителей [43]: лазерное кильватерное ускорение [44], плазменное кильватерное ускорение [45] и структурное кильватерное ускорение [46]. В таких ускорителях можно потенциально получить огромные значения градиентов поля (до $1 \mathrm{TeV} / \mathrm{m}$ [47]), в том числе и для ионов [48]. Однако эти системы имеют существенные недостатки, такие как низкое качество энергетического спектра пучка, крайне малая частота повторения и необходимость иметь громоздкий источник возбуждения кильватерных полей (например, 150 TW лазер) [49].

Значительный прогресс в миниатюризации кильватерных ускоряющих структур осуществлен в рамках инициативы „ускоритель на чипе“ (рис. 2), основанной на возбуждении в диэлектрической структуре полей с градиентом до $1 \mathrm{GV} / \mathrm{m}$ [50]. Однако данная разработка имеет ряд нерешенных практических вопросов, таких как инжекция пучка, фокусировка, получение больших токов, а также компактность лазерной установки [51]. 
Тем не менее стоит отметить эту работу как тенденцию увеличения рабочей частоты ускоряющих структур вплоть до оптических диапазонов.

Тенденция использования высокоградиентных структур не ограничивается импульсными линейными ускорителями электронов, но распространяется также и на ионные ускорители, в том числе циклические, такие как кольцевой фазотрон или немасштабируемый FFAG [52], который требует значительного прироста энергии за оборот для реализации, так называемого „серпантинного ускорения“ [53]. Протонный FFAG непрерывного действия на энергию $900 \mathrm{MeV}$ с токами пучка порядка 1-10 mA, необходимыми для систем трансмутации ядерных отходов или субкритических ядерных реакторов (Accelerator Driven Systems, ADS) [54], требует для своей работы прироста энергии в $20 \mathrm{MeV}$ за оборот, что стало возможным только благодаря прогрессу в развитии ускорительных технологий [55]. В качестве альтернативы дорогим и сложным сверхпроводящим ускорителям систем ADS реакторов предлагается использовать более простые, надежные и низкоэнергетические электронные линейные ускорители, разрабатываемые в Харьковском физико-технический институте (ХФТИ) и в Научноисследовательском институте электрофизической аппаратуры им. Д.В. Ефремова (НИИЭФА) [56,57].

\section{2. Вариативность}

Помимо тенденции к миниатюризации, современные прикладные ускорители также имеют тренд на вариативность параметров ускоренного пучка. Зачастую такая перестройка нужна непосредственно во время работы и часто с изменением от импульса к импульсу. Примером систем с такими требованиями являются комплексы инспекции грузов, основанные на современных методах радиографии [58], например, адаптивные методы инспекции [59]. В этих системах пучок электронов должен изменять энергию и интенсивность внутри одного СВЧ импульса, что позволяет повысить скорость и точность получения изображения с информацией об элементном составе исследуемого объекта (так называемый Zанализ [60]) по сравнению с традиционными моно- и дуоэнергетическими методами радиографии [61].

Другим примером необходимости изменения параметров пучка от импульса к импульсу является ускоритель ионов (протонов или ионов углерода) для облучения опухолей [62]. Здесь с помощью перестройки энергии и синхронизации ионного пучка с системой сканирования (с частотой до $1 \mathrm{kHz}$ ) возможно добиться эффективного лечения движущихся опухолей [63]. Поскольку энергия пучка определяет глубину его проникновения, быстрое изменение энергии позволяет лечить опухоль по принципу трехмерного принтера [64] в течение нескольких минут [65].

Похожий принцип наблюдается и в промышленных системах для облучения материалов [66]. Например, ускорители для вулканизации каучука должны менять энергию в пределах $1-4 \mathrm{MeV}$ для того, чтобы получить оптимальную глубину проникновения в резиновый латекс на 30-130 mm [67]. Наконец, вариативность параметров ускорителя требуется для радиационной закалки и тестирования электронных компонентов. С помощью ускорителя с переменными параметрами можно воссоздать условия, идентичные тем, что наблюдаются в космосе [68].

\section{3. Экономическая эффективность}

Наиболее очевидная на первый взгляд тенденция в коммерческих ускорителях, а именно снижение их стоимости, в том числе эксплуатационной, на самом деле не так однозначна. В широком смысле требования к параметрам ускорителя, их надежности и качеству пучка более весомы, чем требования к цене, так как время простоя коммерческой установки может достигать стоимости ускорителя. Например, простой ускорителя для онкотерапии в госпиталях США может достигать до сотни тысяч долларов в сутки [69], что сравнимо с его стоимостью.

Одно из главных требований к современным прикладным ускорителям - надежность - может определяться как средняя наработка на отказ $\sim 400 \mathrm{~h}$ при коэффициенте технического использования $\sim 0.9$ [70]. Из-за технических поломок стоимость эксплуатации существенно увеличивается. В настоящее время перспективными моделями можно считать ускорители пусть и по высоким ценам, но с минимальными эксплуатационными издержками. Новые требования к перспективным ускорителям еще более жесткие: средняя наработка на отказ более $500 \mathrm{~h}$ при коэффициенте технического использования более 0.95 . Надежность работы ускорителя в большей степени определяется временем эксплуатации его компонентов: источников частиц, ВЧ-питания, вакуумных окон и других ВЧ-компонентов, а так же электроники и криогенных систем (в сверхпроводящих ускорителях). Такие эффекты, как ВЧ-пробой или квенч, также могут вывести ускоритель из строя или навсегда ухудшить его параметры. В этом случае разработка высокоградиентных структур также способствует увеличению надежности установки.

Массовые серии современных промышленных ускорителей также должны быть приспособлены для персонала без высшего образования. Ремонтопригодность перспективных ускорителей должна соответствовать нормам, аналогичным принятым в авиа- и автомобилестроении и позволять проводить полную компьютерную диагностику.

В то же время цена коммерческих ускорителей на сформировавшихся рынках уже достаточно низка по сравнению с другими компонентами систем на их основе, или их эксплуатацией. Тем не менее существует ряд случаев, когда снижение цены ускорителя необходимо. К таким случаям можно отнести: 

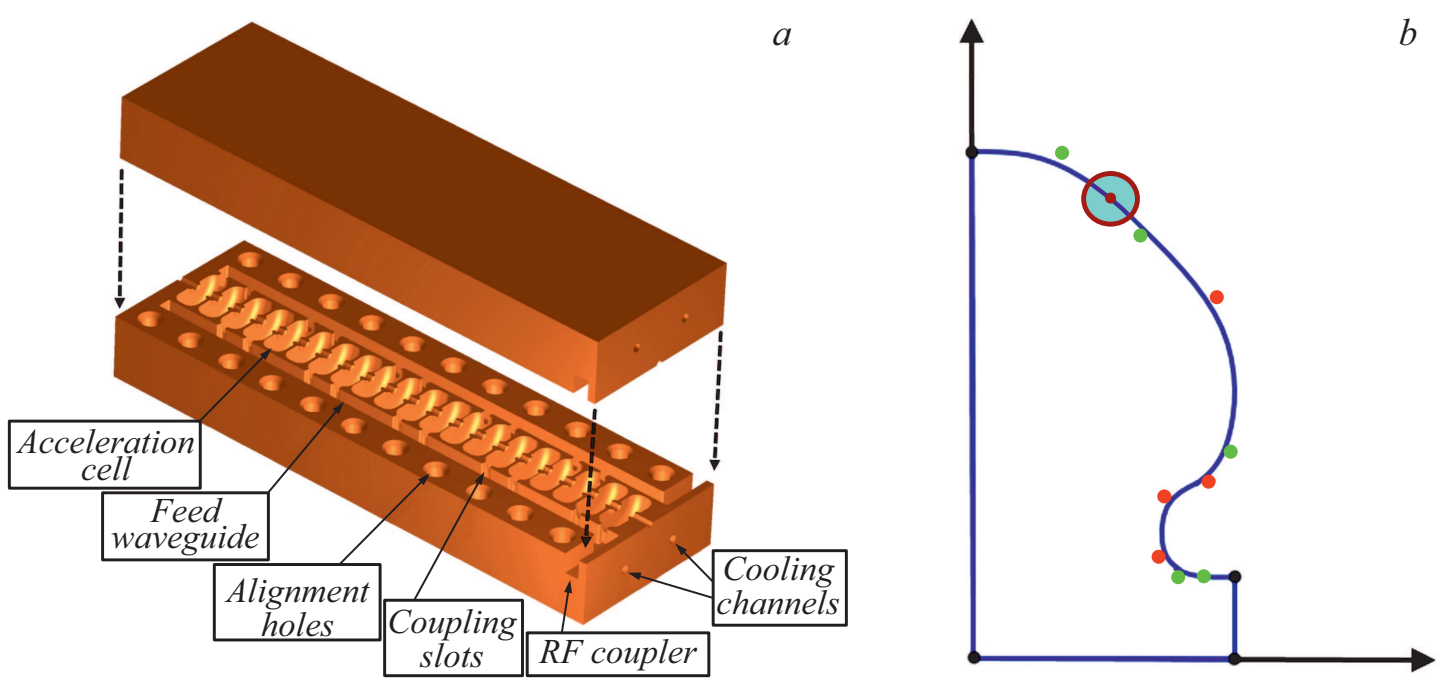

Рис. 3. Пример одного из перспективных направлений развития ускоряющих структур: разделенная ускоряющая структура с распределенным питанием ячеек $(a)$, разработанная в Стэнфордской Национальной Ускорительной Лаборатории SLAC, CША. Отсутствие ячеек связи позволяет реализовать сложные формы ускоряющих ячеек, оптимизированных с помощью генетических алгоритмов $(b)$. Структура также может быть выполнена из новых сплавов, таких, как $\mathrm{CuAg}$, и сварена с помощью технологии электронно-лучевой сварки вместо традиционной пайки.

• ускорители, предназначенные для замены недорогих радиоактивных источников;

• ускорители для радиотерапии в странах „третьего мира";

- ускорители для применения в системах с высокой скоростью обработки, таких, как обеззараживание продуктов, воды, стерилизация или промышленное облучение материалов, где требуется снижение эксплуатационных расходов при максимальной эффективности (КПД) системы [71].

Одним из путей оптимизации стоимости ускорителей является использование новых производственных технологий, например, технология послойного синтеза [72] или механическая микрообработка [73]. Кроме этого, появился ряд новых подходов к изготовлению ускоряющих структур, таких как „разделенная“ структура [74] или структура с распределенным питанием ячеек (рис. 3). Последняя была предложена в конце 70-х, годов [75] и исследована в Институте химической кинетики и горения РАН (Новосибирск) [76], но получившая значительный импульс в развитии благодаря современным разработкам в Стэнфордской ускорительной лаборатории SLAC [77].

Конструктивная особенность разделенной структуры заключается в том, что вместо традиционного изготовления множества индивидуальных ускоряющих ячеек, которые затем должны быть спаяны и настроены, структура изготавливается из двух половинок, в каждой из которой выточен профиль всех ячеек, которые затем либо традиционно спаиваются, либо соединяются с помощью новых методов электронно-лучевой сварки [78] или диффузионного соединения [79]. Также идут работы по разработке структур, не требующих пайки благодаря соединению через острые кромки [80]. Такие методы позволяют достичь более высокой точности изготовления, уменьшить количество составных элементов, избежать спаек в областях, чувствительных для СВЧ-параметров, а в случае использования альтернативных пайке методов соединения получить ускоритель из неотожженой меди, свойства которой более благоприятны для работы на высоких градиентах [81]. Наш опыт работы с разделенными структурами свидетельствует о потенциальном исключении этапа настройки в производстве таких ускорителей [82].

Принцип работы структуры с распределенным питанием ячеек основан на отсутствии электромагнитной связи между соседними ячейками и индивидуальном подводе мощности, что, во-первых, позволяет исключить ячейки связи из такой структуры, необходимые для стабильной работы традиционных структур со связанными резонаторами [83], а во-вторых, оптимизировать форму ячеек с помощью генетических алгоритмов [84]. Таким образом, удается значительно повысить энергетическую эффективность (шунтовое сопротивление) таких структур по сравнению с традиционными [85]. Основным преимуществом подобной структуры является стабильная работа в режиме высоких градиентов, когда в случае ВЧ-пробоя из строя выходит одна ускоряющая ячейка, что не приводит к эффекту обрыва импульса тока во всей секции. К недостаткам таких структур можно отнести чрезмерную сложность изготовления, однако благодаря использованию технологии разделенной структуры эту сложность удалось в значительной степени минимизировать.

Интересно отметить применение в ускорительной технике новых сплавов на основе неотожженной ме- 
ди, таких как $\mathrm{CuAg}, \mathrm{CuCr}, \mathrm{CuZr}$, которые способны выдержать локальный перепад температур в течение импульса до $110^{\circ} \mathrm{C}$ по сравнению с $50^{\circ} \mathrm{C}$ для традиционной отожженной меди [86], а значит, и работать при более высоких градиентах ВЧ-полей. Ожидается, что перспективные материалы для будущих высокоградиентных ускорителей будут иметь высокий предел прочности (> $300 \mathrm{MPa})$, высокую проводимость (> 95\% от значения международного стандарта для отожженной меди IACS [87]), и сохранять свои свойства до $500^{\circ} \mathrm{C}$ при работе в высоком вакууме до $10^{-9}$ Torr [88]. Стоит отметить, что все вышеперечисленные инновационные подходы могут быть скомбинированы для достижения максимальной эксплуатационной и производственной эффективности.

Наконец, отдельным классом стоят ускорители непрерывного действия. Существует ряд задач, где импульсный режим либо неприменим (например, в системах инспекции на быстрых нейтронах [89]), либо неэффективен из-за малой величины скважности. Примером таких задач является использование ускорителей в системах с высокой интенсивностью облучения, таких как вулканизация полимеров или полимеризация мономеров с образованием привитых полимеров (полиэтилена, полипропилена, фторполимеров), где требуется непрерывное облучение пучком $10 \mathrm{MeV}$ электронов с дозами 30-150 kGy [90-94]. Облучение пищевых продуктов проводится дозами $0.1-50 \mathrm{kGy}$ в зависимости от конкретного применения [95,96]. Дезинфекция сточных вод требует облучения до 100000 тонн воды в день дозой $0.2-15 \mathrm{kGy}$ и выше [97].

Для получения непрерывных пучков больших мощностей (до $1 \mathrm{MW}$ ) предпочтительно использование сверхпроводящих ускорителей [98] благодаря малой мощности рассеяния в таких структурах [99]. Однако традиционные сверхпроводящие ускорители являются довольно громоздкими, дорогими и сложными в эксплуатации системами, что обусловлено сложностью криогенной системы для получения жидкого гелия [100]. В последнее время эта проблема начала решаться благодаря развитию компактных рефрижераторов [101], работающих по циклу Гиффорда-Мак-Магона [102], или охладителей на пульсирующих трубках [103]. Мощность таких рефрижераторов достигла уровня, допускающего разработку сверхпроводящих ускорителей, не требующих подключения к внешней системе охлаждения, а значит, более дешевых в эксплуатации и пригодных к использованию в мобильных системах. Инновационный подход таких ускорителей включает в себя использование современных сверхпроводящих материалов с более низкими ВЧ-потерями, таких как станнид триниобия $\mathrm{Nb}_{3} \mathrm{Sn}$ [104] или диборид магния $\mathrm{MgB}_{2}$ [105], а также применение безжидкостных схем охлаждения сверхпроводящих резонаторов [106], что позволяет значительное упростить разработку криомодулей и снизить эксплуатационные расходы.

\section{2. Перспективные направления использования ускорителей}

В следующих разделах будет дано более подробное описание наиболее интересных с точки зрения автора примеров прикладного применения ускорителей и используемых для этого технологий, которые наглядно демонстрируют описанные выше тенденции, и применение современных технологий. К таким направлениям можно отнести ускорители для систем инспекции грузов, материалов и ядерных объектов, медицинские ускорители, а также промышленные системы, в которых требуется замена радиоактивных источников на альтернативные технологии. В табл. 1 приведены основные типы перспективных ускорителей, рассмотренных в настоящей работе, а также технологии с конкретным требованием к ускорителю (миниатюризация, вариативность, эффективность). Кроме того, дается краткая информация о параметрах пучка, исходя из заявленных требований.

\section{1. Ускорители для инновационных систем инспекции}

Обнаружения специальных ядерных материалов может осуществляться как пассивными, так и активными методами. Пассивные методы инспекции основаны на обнаружении гамма частиц с энергией $186 \mathrm{keV}$, что соответствует распаду ${ }^{235} \mathrm{U}$ [108] и $2.60 \mathrm{MeV}$, соответствующие включениям ${ }^{208} \mathrm{Tl}$ [109]. На практике эти методы обладают невысокой эффективностью обнаружения, так как обе эти линии легко экранируются или в другом случае неотличимы от фоновых уровней [110]. Современные активные радиографические системы инспекции грузов на основе ускорителей можно разделить на три категории [111]:

- трансмиссионная рентгеновская радиография с помощью тормозного излучения, полученного с помощью конверсии высокоэнергетического (до $9 \mathrm{MeV}$ [112]) пучка электронов на мишени. Такие системы наиболее распространены, но позволяют получать только информацию о плотности груза в контейнере [113];

- системы инспекции на основе дуальной радиографии [63], когда объект облучается двумя последовательными импульсами с разными энергиями (обычно 4-9 MeV) [114]. Такие системы позволяют получать информацию об эффективном атомном номере $Z$ исследуемого вещества (так называемый $Z$-анализ). Наилучшие результаты в таких системах достигаются при обнаружении тяжелых элементов (так называемых специальных ядерных материалов [115]);

- системы на обратном рассеянии [116]. Эти системы основаны на эффекте обратного рассеивания рентгеновских лучей на исследуемом объекте, и используют низкоэнергетичные пучки фотонов $(\sim 0.25 \mathrm{MeV})$. Такой метод наиболее эффективен для обнаружения органических веществ (взрывчатки, наркотиков), однако 
Таблица 1. Сравнение требований и технологий для перспективных промышленных систем, основанных на резонансных ускорителях

\begin{tabular}{|c|c|c|c|c|}
\hline Класс применения & Пример системы & Основное требование & Основные параметры пучка & Новые технологии \\
\hline 1 & 2 & 3 & 4 & 5 \\
\hline \multirow[t]{5}{*}{$\begin{array}{l}\text { Инспекционные } \\
\text { ускорители }\end{array}$} & $\begin{array}{c}\text { Адаптивная } \\
\text { стационарная }\end{array}$ & $\begin{array}{c}\text { Вариативность: } \\
\text { изменение энергии } \\
\text { пучка в течение } \\
\text { импульса }\end{array}$ & $\begin{array}{c}\text { Энергия } \gamma 2-9 \mathrm{MeV}, \\
\text { длина импульса } 16 \mu \mathrm{s}, \\
\text { частота повторения } 1000 \mathrm{~Hz}\end{array}$ & $\begin{array}{c}\text { Линейные ускорители } \\
\text { с эффективной группировкой } \\
\text { частиц, использование } \\
\text { эффективных СВЧ источников } \\
\text { (многолучевые клистроны, } \\
\text { амплитроны и т.д.) }\end{array}$ \\
\hline & Мобильная & $\begin{array}{c}\text { Компактность: } \\
\text { способность } \\
\text { размещаться на } \\
\text { коммерческом шасси }\end{array}$ & $\begin{array}{c}\text { Энергия } \gamma 2-6 \mathrm{MeV}, \\
\text { доза облучения } \\
75 \mathrm{cGy} / \mathrm{min}\end{array}$ & $\begin{array}{c}\text { Использование магнетронов } \\
X \text {-частотного диапазона, } \\
\text { использование выско- } \\
\text { градиентных структур }\end{array}$ \\
\hline & Переносная & $\begin{array}{l}\text { Компактность: } \\
\text { возможность } \\
\text { транспортировки } \\
\text { одним человеком }\end{array}$ & Энергия $\gamma$ до $1 \mathrm{MeV}$ & $\begin{array}{c}\text { Использование магнетронов } \\
\text { Ки-частотного диапазона } \\
\text { и разделенных структур }\end{array}$ \\
\hline & $\begin{array}{l}\text { На основе } \\
\text { ядерных } \\
\text { реакций }\end{array}$ & $\begin{array}{c}\text { Компактность: } \\
\text { возможность транс- } \\
\text { портировки между } \\
\text { центрами размещения } \\
\text { Вариативность: } \\
\text { получение „коктейля“ } \\
\text { заряженных частиц [107] }\end{array}$ & $\begin{array}{c}\text { Непрерывные пучки } \\
\text { ионов от водорода до } \\
\text { кальция до энергий } \\
15-20 \mathrm{MeV} / \mathrm{n} \\
\text { и интенсивностью } \\
\text { до } 1 \mathrm{~mA}\end{array}$ & $\begin{array}{c}\text { Использование } \\
\text { высокотемпературных } \\
\text { сверхпроводящих } \\
\text { материалов }\end{array}$ \\
\hline & $\begin{array}{l}\text { Исследование } \\
\text { ядерного } \\
\text { топлива }\end{array}$ & $\begin{array}{c}\text { Вариативность: } \\
\text { возможность облучения } \\
\text { различными ионами }\end{array}$ & $\begin{array}{c}\text { Непрерывные пучки } \\
\text { ионов от водорода } \\
\text { до урана до энергий } \\
1.5 \mathrm{MeV} / \mathrm{n}\end{array}$ & $\begin{array}{c}\text { Использование } \\
\text { эффективных } \\
\text { выскоградиентных } \\
\text { нормальнопроводящих } \\
\text { ускорителей }\end{array}$ \\
\hline \multirow[t]{4}{*}{$\begin{array}{l}\text { Замена } \\
\text { радиоизотопов }\end{array}$} & $\begin{array}{c}\text { Ядерная } \\
\text { безопасность } \\
(\text { Со-57) }\end{array}$ & $\begin{array}{l}\text { Компактность: } \\
\text { возможность } \\
\text { транспортировки } \\
\text { одним человеком }\end{array}$ & $\begin{array}{c}\text { Энергия } \gamma \text { до } 180 \mathrm{keV}, \\
\text { доза около } 1 \mathrm{mCi}, \\
\text { частота повторения } \\
\text { импульсов }>4 \mathrm{kHz}\end{array}$ & $\begin{array}{c}\text { Использование } \\
\text { магнетронов } \\
K u \text {-частотного диапазона } \\
\text { и разделенных структур }\end{array}$ \\
\hline & $\begin{array}{c}\text { Выездная } \\
\text { радиография } \\
\text { (Ir-192) }\end{array}$ & $\begin{array}{c}\text { Эфбективность: } \\
\text { получение необходимой } \\
\text { дозы при ограниченных } \\
\text { размерах }\end{array}$ & $\begin{array}{l}\text { Энергия } \sim 1 \mathrm{MeV}, \\
\text { доза } \sim 200 \mathrm{Ci}\end{array}$ & $\begin{array}{c}\text { Использование } \\
\text { диэлектрических } \\
\text { структур с малыми } \\
\text { потерями }\end{array}$ \\
\hline & $\begin{array}{c}\text { Гамма-каротаж } \\
\text { скважин } \\
(\mathrm{Cs}-137)\end{array}$ & $\begin{array}{c}\text { Компактность: } \\
\text { возможность работы } \\
\text { в узкой скважине }\end{array}$ & $\begin{array}{c}\text { Энергия } \sim 2.5 \mathrm{MeV}, \\
\text { средняя } \\
\text { мощность }>5 \mathrm{~W}\end{array}$ & $\begin{array}{c}\text { Использование структур, } \\
\text { совмещенных с ВЧ } \\
\text { источником (т.н. клилаки) }\end{array}$ \\
\hline & $\begin{array}{c}\text { Дефектоскопия } \\
(\text { Со-60) }\end{array}$ & $\begin{array}{c}\text { ЭЯфективность: } \\
\text { стоимость, сравнимая } \\
\text { с радиоизотопами }\end{array}$ & $\begin{array}{l}\text { Энергия } \sim 4 \mathrm{MeV}, \\
\text { доза > } 50 \mathrm{cGy} / \mathrm{min}\end{array}$ & $\begin{array}{c}\text { Использование магнетронов } \\
X \text {-частотного диапазона, } \\
\text { использование выскогра- } \\
\text { диентных структур }\end{array}$ \\
\hline \multirow[t]{2}{*}{ Радиотерапия } & $\begin{array}{l}\text { Фотонная } \\
4 \pi \text {-терапия }\end{array}$ & $\begin{array}{c}\text { Компактность: } \\
\text { необходимость вращать } \\
\text { ускоритель на телесный } \\
\text { угол } 4 \pi \text { вокруг пациента }\end{array}$ & $\begin{array}{l}\text { Энергия } \gamma \sim 3-6 \mathrm{MeV}, \\
\quad \text { доза } 5-10 \mathrm{~Gy} / \mathrm{min}\end{array}$ & $\begin{array}{c}\text { Использование магнетронов } \\
X \text {-частотного диапазона, } \\
\text { использование выскогра- } \\
\text { диентных структур }\end{array}$ \\
\hline & $\begin{array}{c}\text { Фотонная } \\
\text { FLASH-терапия }\end{array}$ & $\begin{array}{c}\text { Эфбективность: } \\
\text { доставка очень большой } \\
\text { дозы в течение } \\
\text { короткого времени }\end{array}$ & $\begin{array}{c}\text { Энергия } \gamma \sim 1-12 \mathrm{MeV}, \\
\text { доза > 50 Gy в течение } \\
\text { нескольких секунд }\end{array}$ & $\begin{array}{c}\text { Использование компактных } \\
\text { и эффективных СВЧ источников } \\
\text { (многолучевые клистроны), } \\
\text { использование разделенных } \\
\text { и распределенных структур }\end{array}$ \\
\hline
\end{tabular}


Таблица 1 (продолжение).

\begin{tabular}{|c|c|c|c|c|}
\hline 1 & 2 & 3 & 4 & 5 \\
\hline & $\begin{array}{c}\text { Адронная } \\
\text { терапия }\end{array}$ & $\begin{array}{c}\text { Вариативность: } \\
\text { изменение энергии } \\
\text { пучка от импульса } \\
\text { к импульсу для } \\
\text { трехмерного облучения } \\
\text { опухоли } \\
\text { Эябективность: } \\
\text { снижение стоимости } \\
\text { установки до значений, } \\
\text { приемлемых для } \\
\text { массового использования }\end{array}$ & $\begin{array}{c}\text { Энергия протонов } \\
\text { до } 250 \mathrm{MeV}, \text { ионов } \\
\text { углерода до } 450 \mathrm{MeV} \\
\text { ток } \sim \mathrm{nA}, \text { частота } \\
\text { повторения импульсов } \\
\text { до } 1000 \mathrm{~Hz}\end{array}$ & $\begin{array}{c}\text { Использование } \\
\text { высокоградиентных } \\
\text { нормальнопроводязщих } \\
\text { структур с малыми } \\
\text { значениями фазовой } \\
\text { скорости, возможно с } \\
\text { охлаждением жидким } \\
\text { азотом/неоном }\end{array}$ \\
\hline \multirow[t]{2}{*}{$\begin{array}{l}\text { Промышленное } \\
\text { облучение } \\
\text { материалов }\end{array}$} & $\begin{array}{c}\text { Обеззараживание } \\
\text { сточных вод }\end{array}$ & $\begin{array}{c}\text { Эوбективность: } \\
\text { получение промышленных } \\
\text { доз облучения } \\
\text { при минимизации } \\
\text { эксплуатационных } \\
\text { расходов }\end{array}$ & $\begin{array}{c}\text { Энергия электронов } \\
1-10 \mathrm{MeV}, \text { средняя } \\
\text { мощность пучка } \\
0.1-10 \mathrm{MW}\end{array}$ & $\begin{array}{c}\text { Использование } \\
\text { высокодобротных } \\
\text { сверхпроводящих резонаторов } \\
\text { (электрополировка, допинг } \\
\text { атомов азота, быстрое } \\
\text { охлаждение и т. д.), } \\
\text { компактных криокулеров } \\
\text { большой мощности } \\
\text { и технологи безжидкостного } \\
\text { охлаждения }\end{array}$ \\
\hline & $\begin{array}{l}\text { Производство } \\
\text { радиоизотопов }\end{array}$ & $\begin{array}{c}\text { Эوфбективность: } \\
\text { получение большого } \\
\text { количества радиоизотопов } \\
\text { при снижении их стоимости }\end{array}$ & $\begin{array}{c}\text { Энергия протонов или } \\
\alpha \text {-частиц 20-80 MeV, } \\
\text { средний ток }>1 \mathrm{~mA}\end{array}$ & $\begin{array}{c}\text { Использование изохронных } \\
\text { синхроциклотронов } \\
\text { типа FFAG, с высокогра- } \\
\text { диентными резонаторами }\end{array}$ \\
\hline
\end{tabular}

Пр имечан и е. * Здесь и далее: доза на расстоянии $1 \mathrm{~m}$ от ускорителя.

имеет невысокую глубину проникновения в отличие от высокоэнергетичных трансмиссионных методов.

Несмотря на достаточное развитие данных систем, они имеют существенные недостатки. Традиционный метод дуальной радиографии имеет серьезные ограничения по скорости получения изображения (до $15 \mathrm{~km} / \mathrm{h}$ ), обусловленное необходимостью облучать груз парными пучками с разными энергиями. В таком случае среднее время получения одного пикселя изображения составляет порядка $5 \mathrm{~ms}$, что обусловлено периодом следования ВЧ импульсов в ускорителе (обычно, около $2.5 \mathrm{~ms}$ ). Кроме того, временное разделение соседних импульсов с разными энергиями вырождается в пространственное разделение из-за движения грузов, что приводит к размыванию пикселя изображения (рис. 4, a). Таким образом, необходимо замедлять движение объекта до $15 \mathrm{~km} / \mathrm{h}$. При этом фиксированный выбор значений энергий имеет наивысшую эффективность распознавания только для определенного диапазона значений $Z$ материала [117]. Наконец, спектр тормозного излучения является сплошным [118] (до коротковолновой границы), что также снижает точность восстановления изображения.
В следующем разделе будет рассмотрено несколько примеров инновационных активных методов исследования, которые требуют разработки новых источников излучения (ускорителей) для своей реализации.

\subsection{1. Адаптивные методы инспекции}

Для решения этих проблем было предложено несколько новых методов инспекции: дуальные методы на основе сцинтилляционно-черенковских детекторов [119], мультиэнергетические адаптивные методы на основе вариации энергии пучка внутри одного ВЧ-импульса [120], адаптивные методы для систем на обратном рассеянии [121], а также использование источников на основе обратного комптоновского излучения для получения квазимонохроматических пучков фотонов [122].

Как упоминалось ранее, в адаптивных методах каждый пучок рентгеновского излучения длительностью, равной длительности ВЧ-импульса, состоит из последовательности коротких микроимпульсов длиной 250-400 ns, разделенных короткими интервалами в 50-150 ns [123], как показано на рис. $4, b$. Ключевым преимуществом этого метода является то, что $Z$-анализ происходит для одного пространственного положения исследуемого объекта, 

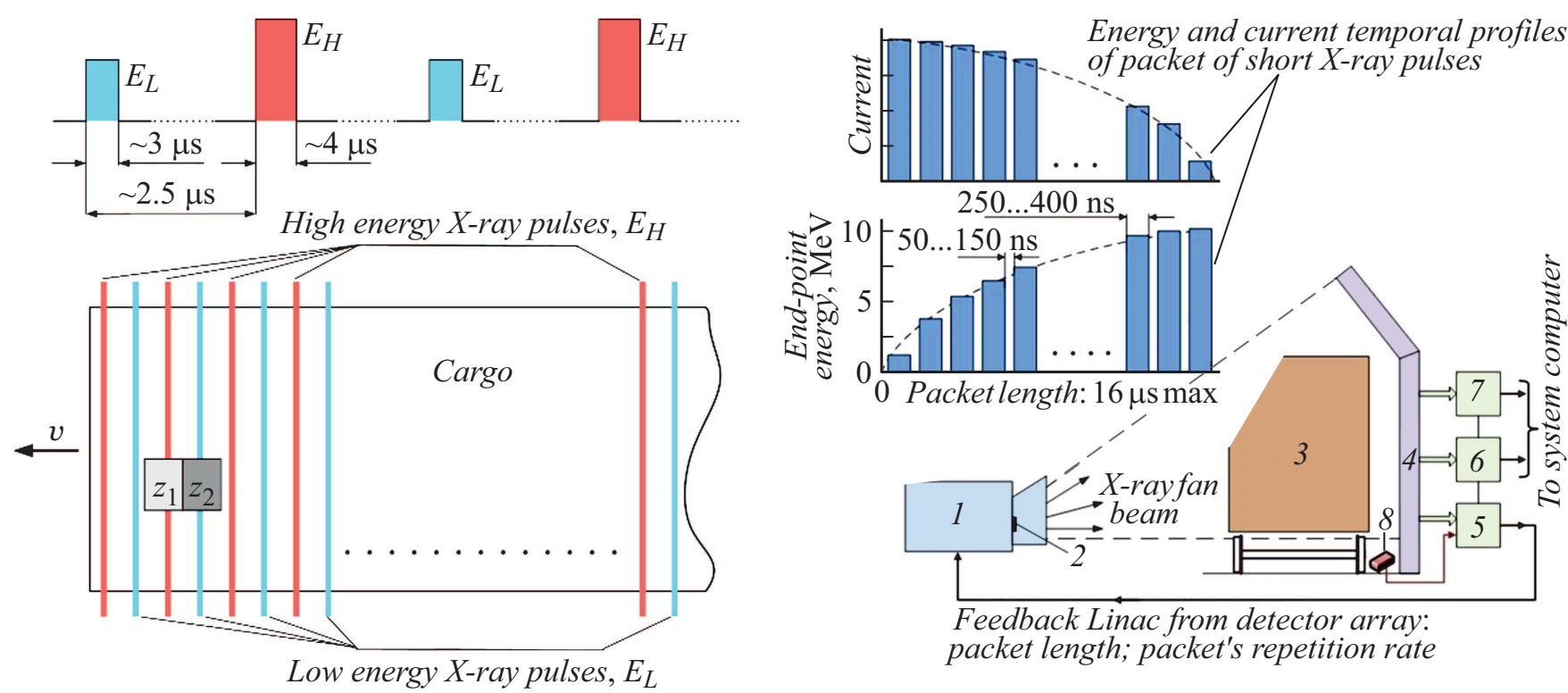

Feedback Linac from detector array: packet length; packet's repetition rate

Рис. 4. Временна́я диаграмма высоко- (красным) и низко- (синим) энергетичных импульсов в системе, на основе дуального (a) и адаптивного $(b)$ методов инспекции; 1 - ускоритель электронов, $2-$ конвертор тормозного излучения, $3-$ инспектируемый груз, 4 - массив детекторов, 5-7 - системы контроля и обработки изображения. Рисунки А. Ародзеро, RadiaBeam Technologies (https://radiabeam.com).

Таблица 2. Требования, предъявляемые к ускорителям электронов для адаптивных методов инспекции в зависимости от области их применения

\begin{tabular}{l|c|c|c|c}
\hline \multicolumn{1}{c|}{ Тип системы } & $\begin{array}{c}\text { Диапазон изменения } \\
\text { энергий, } \mathrm{MeV}\end{array}$ & $\begin{array}{c}\text { Доза облучения на один } \\
\text { линейный пиксель, cGy }\end{array}$ & $\begin{array}{c}\text { Частота следования пакетов } \\
\text { микроимпульсов, Hz }\end{array}$ & $\begin{array}{c}\text { Длина пакета } \\
\text { импульсов, } \mu \mathrm{s}\end{array}$ \\
\hline Инспекция быстродвижущихся & & & & \\
грузов (ж/д) & $2-9$ & 0.125 & 1000 \\
Стационарная & $2-6$ & 0.055 & 400 & 8 \\
Мобильная & $2-7.5$ & 0.028 & 2000 & 0.5 \\
Мобильная (обратное рассеяние) & $0.5-1.5$ & - & 4000 & 8 \\
Компактная (посылки, конверты) & $0.2-1$ & - & 1000 & 4
\end{tabular}

что значительно повышает контрастность изображения. Кроме того, на основе полученных данных система выбирает оптимальный диапазон энергий для следующего пакета микроимпульсов (адаптивность). Этот метод может также применяться для адаптивных систем на обратном рассеянии и компьютерной томографии [26].

В зависимости от конкретных применений системы, таких как стационарная система инспекции железнодорожных составов, мобильная система, установленная на подвижное шасси некоммерческого типа [124], компактная система для инспекции посылок и т.д., к ускорителям предъявляются разные требования (табл. 2), в частности, по диапазону перестройки энергии и необходимой дозе излучения, обусловленной в первую очередь толщиной контейнера и глубиной проникновения пучка [125].
В соответствии с данными требованиями был разработан ряд новых ускорителей. Ускорители для системы адаптивной инспекции железнодорожных составов и мобильной досмотровой установки показаны на рис. 5. С их детальным техническим описанием можно ознакомиться в работах $[26,126]$. На примере этих двух ускорителей можно видеть необходимость в вариативности энергии пучка для реализации адаптивного метода и миниатюризации для размещения на мобильном шасси. Вариативность параметров пучка обеспечивается специальным дизайном группирователя с эффективным захватом частиц в режим ускорения для широкого диапазона рабочих значений ВЧ-мощности, с помощью которого регулируется энергия пучка [127]. Компактность ускорителя достигнута работой на более высоких частотах $(9 \mathrm{GHz})$ благодаря более низким требованиям к верхнему значению 
$a$
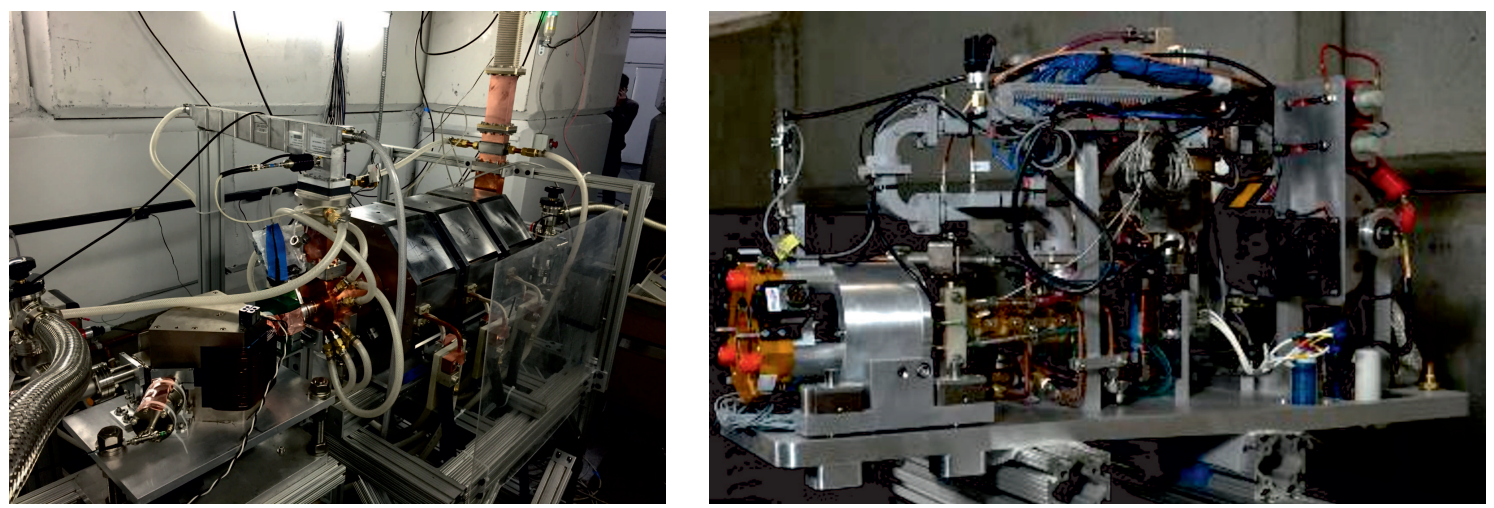

Рис. 5. Линейные ускорители электронов для системы адаптивной инспекции быстродвижущихся $(45 \mathrm{~km} / \mathrm{h})$ железнодорожных составов $(a)$ и мобильной системы инспекции грузов $(b)$, разработанные и созданные в компании RadiaBeam Technologies, CША. Фотографии RadiaBeam Technologies (https://radiabeam.com).

энергии и дозе облучения (более легкие цели инспекции).

Другим примером ускорителя для мобильных систем инспекции может служить бетатрон, принцип действия которого основан на ускорении с помощью вихревого электрического поля [128]. Данный тип ускорителя отличается большей компактностью за счет отсутствия системы ВЧ-питания, однако доза излучения бетатрона намного ниже, чем у линейного ускорителя (около $7.5 \mathrm{cGy} / \mathrm{min}$ ) [129], что тем не менее достаточно для мобильных систем инспекции. Помимо компактности и невысокой производственной и эксплуатационной стоимости, данный ускоритель можно также сделать вариативным с помощью частичной экстракции пучка [130], что также делает его пригодным для перспективных систем инспекции [131].

\subsection{2. Инспекция с помощью непрерывного излучения}

Хотя практически все современные методы инспекции работают в импульсном режиме, некоторые перспективные методы, например системы инспекции на быстрых нейтронах [132], требуют пучков непрерывного действия. Этот принцип основан на измерении потока быстрых $(\sim 2 \mathrm{MeV})$ нейтронов, полученных в результате фотоядерной реакции высокоэнергетичных фотонов с атомами тяжелых элементов (например, актинидов). Новейшие системы, основанные на этом принципе, также имеют требования к ускорителю по перестройке энергии до $10 \mathrm{MeV}$ с шагом в $2-3 \mathrm{MeV}$ и тока от $0.1 \mu \mathrm{A}$ до $1 \mathrm{~mA}$.

Несмотря на то, что сверхпроводящие ускорители могут быть более эффективны в таком режиме, в настоящее время их текущее состояние не позволяет использовать их в мобильных системах инспекции. Другой тип нормальнопроводящего ускорителя непрерывного действия, широко применяемый в промышленности родотрон [133], принцип действия которого построен на принципе рециркуляции пучка через серию последовательных отверстий в коаксиальном резонаторе, не удовлетворяет требованиям вариативности и компактности для использования в мобильных инспекционных комплексах.

Для решения этой проблемы предлагается использовать разрезный микротрон, который может работать в непрерывном режиме [134-136]. Принцип действия микротрона похож на принцип действия классического циклотрона, где величина магнитного поля и частота ускоряющего поля постоянны. Отличие состоит в том, что период обращения сгустка на каждом обороте изменяется так, чтобы каждый раз частицы приходили в ускоряющий зазор в правильной фазе высокочастотного электрического поля [137]. Данный тип ускорителя допускает миниатюризацию за счет использования резонаторов высоких частот, вариативность за счет возможности вывода пучка с низкоэнергетических орбит, а также производственную и энергетическую эффективность за счет применения современных технологий производства ускорителей. На рис. 6 показана схема такого микротрона, основанного ускоряющей секции, работающей на частоте $7.2 \mathrm{GHz}[138]$, и ускоряющая секция, изготовленная по принципу разделенной структуры.

Стоит, однако, отметить, что единственным действующим ускорителем такого типа в настоящее время является каскад разрезных микротронов Института ядерной физики университета г. Майнц [139]. В силу особенности динамики пучка в разрезном микротроне и особенностей работы нормальнопроводящих ускоряющих структур в непрерывном режиме ускоритель такого типа является неприемлемо сложным и громоздким для прикладных целей, а средний ток пучка ограничен сотнями микроампер.

Альтернативным подходом активной инспекции является использование ускоренных пучков тяжелых частиц, которые могут возбуждать ядерные состояния либо напрямую, либо посредством генерации вторичных высо- 


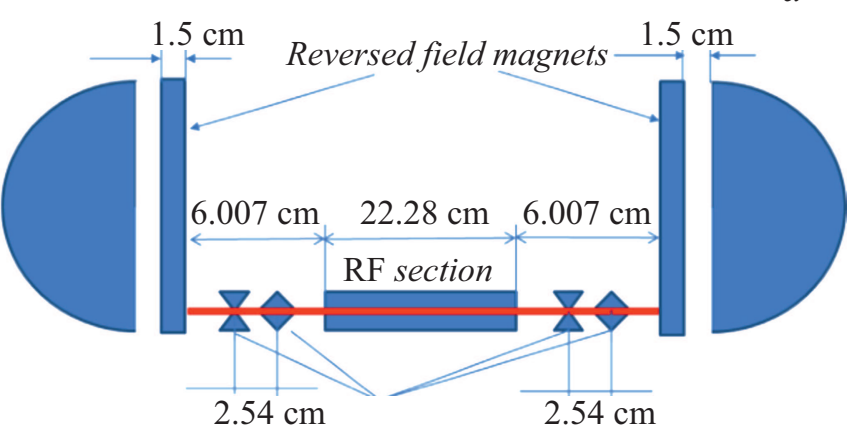

$b$

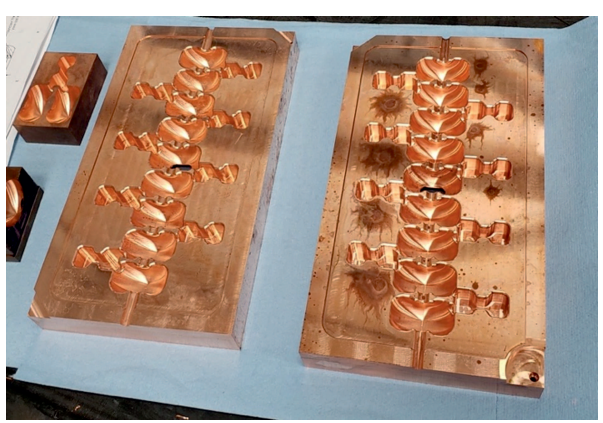

Рис. 6. Схема компактного разрезного микротрона непрерывного действия с переменной энергией $(a)$, основанная на использовании 7.2 GHz ускоряющей структуры разделенного типа (b). Рисунок А.В. Смирнова, RadiaBeam Technologies (https://radiabeam.com).

коэнергетических фотонов или нейтронов, получаемых с помощью ядерных реакций в специальной мишени [140]. В присутствии ядерных материалов такие пучки ионов, фотонов или нейтронов возбуждают характерные состояния ядер, которые могут затем быть выборочно детектированы с помощью методов спектрального поглощения или излучения [141]. Из-за малого сечения реакций и особенности работы детекторов источники тяжелых частиц должны работать в непрерывном режиме.

Изучение ядерных реакций, способных произвести фотоны с энергией $4.4 \mathrm{MeV}$ или $15.1 \mathrm{MeV}$ [142], выдвигают требования к источнику первичного излучения (ускорителя). Такой ускоритель должен выдавать непрерывный поток протонов или дейтронов с энергией до $20 \mathrm{MeV}$ и токами до $1 \mathrm{~mA}$. Например, работы по изучению времени сканирования настоящих грузовых контейнеров с помощью гамма-излучения с энергией $15.1 \mathrm{MeV}$, полученного в результате реакции ${ }^{12} C\left(p, p^{\prime}\right)^{12} C$, показали, что время инспекции с пучком протонов с интенсивностью $1 \mathrm{~mA}$ занимает $60 \mathrm{~s}$ [143].

Наиболее популярным ускорителем протонов на такие энергии является циклотрон благодаря его компактности и эффективности [144]. В мире существует множество коммерческих протонных циклотронов, способных достичь таких значений токов, большинство из которых используют нормальнопроводящие технологии [145]. Тем не менее вес таких ускорителей может достигать десятков тонн, а их стоимость достигать нескольких миллионов долларов. Миниатюризация циклотронов возможна благодаря использованию сверхпроводящих магнитов, с помощью которых можно получать высокие значения магнитных полей, а значит, иметь меньшие радиальные размеры, так как радиус орбиты $R$-частицы с энергией $W$ связан с индукцией магнитного поля $B$ как $W \beta \approx 300 \mathrm{~B} \cdot \mathrm{R}[146]$ (рис. 7, $a$ ). Например, ускоритель протонов до энергии $12 \mathrm{MeV}$ Ionetix 12SC весит всего 2.3 тонны и имеет поперечный размер $44 \mathrm{~cm}$ [147].

Сверхпроводящие циклотроны имеют ряд недостатков. Во-первых, несмотря на кажущуюся энергетическую эффективность, такие ускорители могут потреблять даже больше энергии, чем традиционные, из-за неэффективности криогенной системы [148]. Во-вторых, такие ускорители имеют небольшую интенсивность до десятка $\mu \mathrm{A}$, так как не могут компенсировать большую энергию потерь частиц [149]. При этом из-за малых размеров и небольшого темпа ускорения в таких ускорителях наблюдаются проблемы с разделением орбит и выводом пучка [150].

Стоит также отметить разработку безжелезных синхроциклотронов, использующих магнитные катушки для создания магнитных полей с необходимым для ускорения распределением [151] (рис. 7, $b$ ). Компактность таких систем обусловлена отсутствием железных сердечников. Однако подобные циклотроны могут работать только в импульсном режиме небольшой скважности (до $1 \mathrm{kHz}$ ) из-за необходимости подстройки частоты в процессе ускорения для соблюдения резонансных условий, обусловленной слабыми магнитными полями катушек. Максимально полученный средний ток в таких циклотронах достигает $100 \mathrm{nA}$ [152].

Несмотря на текущие ограничения в работе компактных циклотронов, интерес к таким разработкам очень высок, и тенденция уменьшения размеров и увеличения эффективности хорошо заметны и в этих типах ускорителей.

\subsection{3. Использование монохроматических пучков}

Использование монохроматических пучков фотонов для инспекции грузов имеет несомненные преимущества по сравнению с тормозным излучением - улучшенный контраст и уменьшенная доза облучения [153]. Перспективные схемы инспекции также подразумевают одновременное использование монохроматического излучения нескольких типов, как, например, гамма-излучение с несколькими энергетическими линиями, или одновременное облучение потоков монохроматических фотонов и нейтронов (рис. 8). Такие системы позволяют очень точно измерить среднее значение атомного номера $Z$ исследуемого материала и обнаружить характерные паттерны излучения возбужденных состояний ядер транс- 
$a$

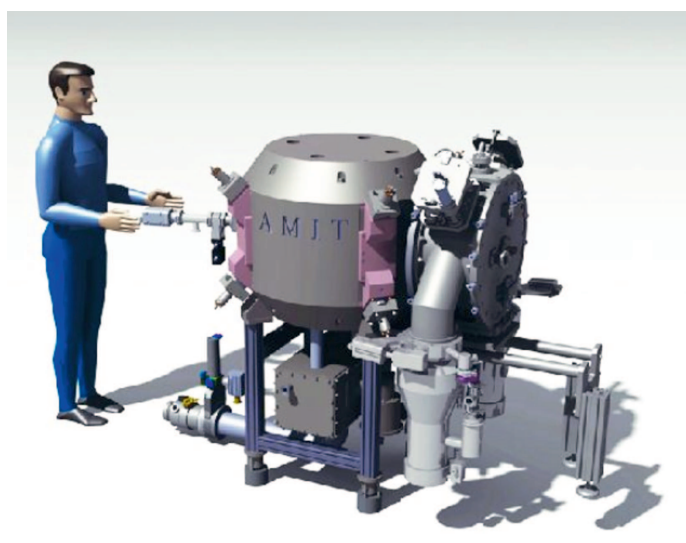

$b$

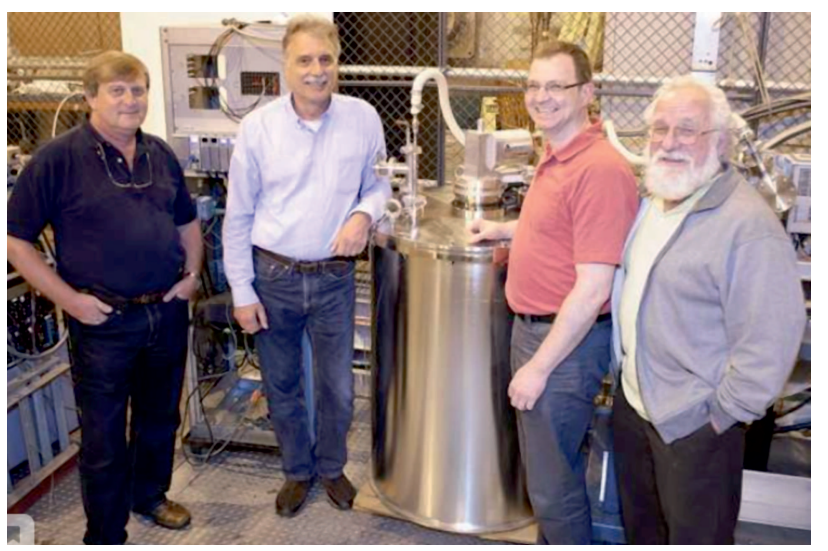

Рис. 7. Примеры компактных сверхпроводящих протонных синхроциклотронов для производства радиоизотопов: $a-$ проекта AMIT до энергии $8.5 \mathrm{MeV}[358,359], b-$ безжелезный циклотрон на энергию $230 \mathrm{MeV}$ [360,361]. Источники фотографий: $a-$ AMIT collaboration (http://www.cenit-amit.com), $b$ - Massachusetts Institute of Technologies (http://www.mit.edu).
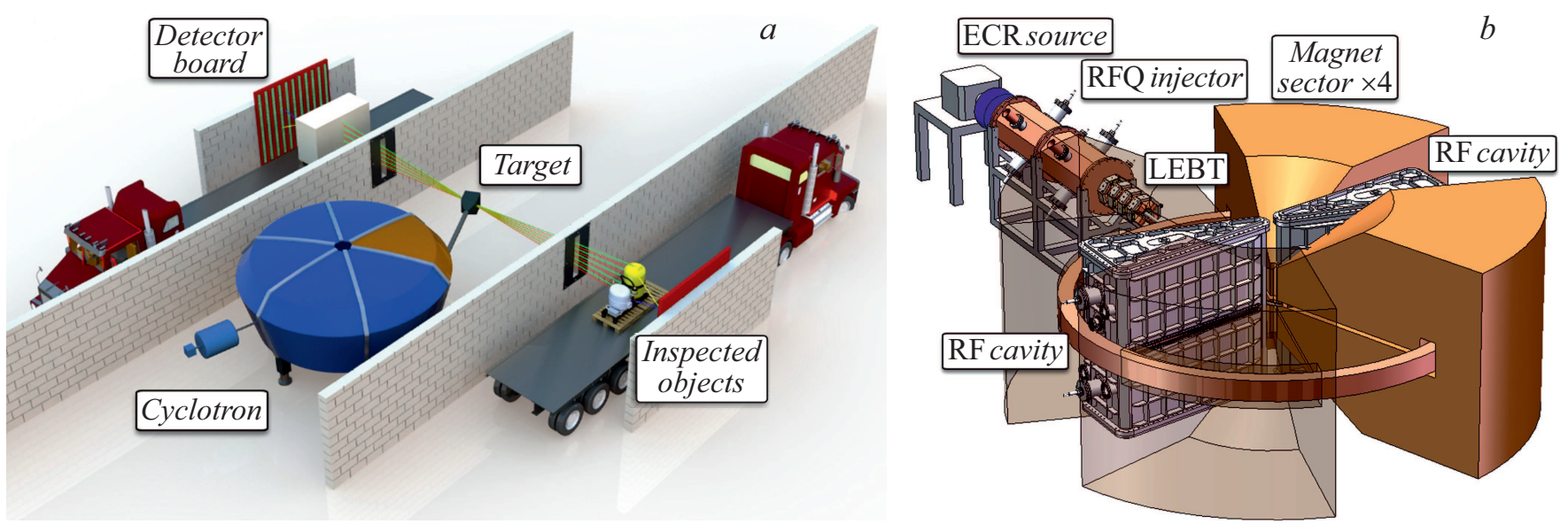

Рис. 8. Нестационарная система инспекции и томографии методом одновременного облучения материалов монохроматическими пучками фотонов и нейтронов $(a)$ на основе компактного секторного изоциклотрона с переменными параметрами $(b)$, разработанная в RadiaBeam Technologies, США.

урановых элементов, однозначно указывающих на наличие специальных ядерных материалов [154,155]. Эти системы также обладают функциями монохроматической томографии без необходимости вращения исследуемого объекта или источника излучения [156].

Ускоритель для систем инспекции на основе ядерных реакций помимо требований, описанных в предыдущей секции, должен обладать значительной вариативностью, заключающейся в возможности ускорять пучки, состоящие из целого набора разных ионов одновременно. На данный момент среди коммерческих укорителей нет компактных циклотронов, обладающих такими свойствами. Перспективные проекты включают в себя разработку секторного изоциклотрона на основе высокоградиентных ВЧ-резонаторов и магнитов с наклонным профилем, позволяющие получать токи пучков любых ионов от водорода до кальция (в том числе одновременно) до $1 \mathrm{~mA}$ с энергиями до 15-20 MeV/nucleon [157]. Размер та- кой системы не будет превышать 3.5-5 m в диаметре, а вес - 50-150 тонн в зависимости от конфигурации, что в 3-6 раз меньше веса протонных циклотронов со сравнимыми параметрами [158].

Наконец, одним из перспективных инструментов для инспекции [159] является источник фотонов, основанный на инверсном Комптоновском рассеянии (ICS) [160], т.е. облучении высокоэнергетичного пучка электронов лазером для получения рассеянных фотонов жесткого рентгеновского диапазона. Этот тип источника позволяет получить компактный (по сравнению с синхротроном), квазимонохроматический (по сравнению с тормозным излучением) пучок фотонов с регулируемой энергией. С его помощью можно эффективно определять стабильные и радиоактивные нуклиды в составе вещества, сканируя его пучком с разными энергиями и измеряя сигнал вынужденного излучения, уникальный для каждого нуклида [161]. 
Для практической реализации такого источника необходимо решить сложную технологическую задачу, заключающуюся в ускорении пакета сгустков электронов с большими токами и малым размером до энергий в сотни $\mathrm{MeV}$, при этом удовлетворяя требованию компактности. Тем не менее благодаря использованию современных технологий, таких, как эффективные криогенные высокоградиентные структуры с подавлением высших типов волн [162], приводящих к эффекту обрыва импульса тока [163], и гибридные ускоряющие структуры $[164,165]$, удалось добиться значительных успехов в реализации данного типа ускорителя [166]. Имеет смысл также отметить возможность применения источника ICS в медицине [167], метрологии [168] и научных исследованиях $[169,170]$.

\subsection{4. Инспекция материалов ядерных реакторов}

Прогресс в ядерной энергетике требует использования новых материалов для увеличения срока эксплуатации ядерных реакторов, эффективности использования ядерного топлива и разработки перспективных типов реакторов $[171,172]$. Такие материалы должны иметь повышенную устойчивость к тепловым, физическим и радиационным нагрузкам [173]. В реакторах свойства материалов ухудшаются со временем из-за микродефектов, вызванных продуктами ядерного распада топлива [174]. Срок эксплуатации реакторных материалов измеряется десятилетиями, в течение которых их структура претерпевает изменения от нескольких смещений на атом (с.н.а) в корпусе реактора до нескольких тысяч с.н.а. в ядерном топливе [175]. Таким образом, для разработки и тестирования новых материалов необходимо подвергать их облучению, эквивалентному облучению в реакторах.

Традиционный метод такого исследования, заключающийся в помещении образцов материалов в ядерный реактор для облучения нейтронами [176], требует огромного времени и средств, и кроме того, не позволяет полностью понять физику протекающих процессов, необходимую для точного моделирования. Типичные дозы, получаемые в исследовательских реакторах, составляют около 3 с.н.а. в год [177], что соответствует более 30 годам непрерывного эксперимента для достижения доз в 100 с.н.а., необходимых для убедительного доказательства применимости материалов для перспективных реакторов [178]. Эта проблема может быть решена использованием ускорителей тяжелых частиц с энергиями, соответствующим энергиям продуктов распада ядерного топлива в реакторе, и дозами до 25 с.н.а. в $\mathrm{h}$.

Разработка такого ускорителя была проведена в Аргоннской Национальной Лаборатории (ANL), США и включала в себя все современные тенденции ускорительной техники [179] (рис. 9). Благодаря использованию источника ионов с высокой степенью обдирки [180], эффективного дизайна ускорителя типа RFQ с трапецеидальной формой электродов [181,182] и высокоградиентных четвертьволновых резонаторов с несколькими трубками дрейфа удалось добиться компактного размера ускорителя в $16 \mathrm{~m}$ для пучков урана, ускоренных до $285 \mathrm{MeV}$. Снижение ВЧ-потерь в резонаторах позволило избежать использования сверхпроводящих структур, несмотря на непрерывный режим работы ускорителя, и значительно сократить стоимость конструкции благодаря отсутствию сложной и дорогой системы рефрижераторов для жидкого гелия [183]. Факт цены играет большую роль в данном случае в связи с обратной зависимостью вероятности финансирования таких проектов от их стоимости. Наконец, ускоритель позволяет получать пучки любых частиц от протонов до урана с любой энергией до $1.2 \mathrm{MeV} /$ nucleon $(1.6 \mathrm{MeV}$ для протонов), что позволяет использовать эту установку для исследования радиационных процессов в материалах не только для ядерных реакторов, но и для широкого спектра применения [184].

\section{2. Ускорители для замены радиоактивных изотопов}

Радиоизотопы вплоть до настоящего времени являются одним из наиболее используемых источников гамма-излучения. Только в США используется около 5000 устройств, содержащих до 55000 высокоактивных радиоизотопов [185]. Популярность таких источников объясняется компактностью, дешевизной и чистотой энергетического спектра излучения [186]. Тем не менее современная тенденция, заданная МАГАТЭ, направлена на замену таких источников альтернативными технологиями, в связи с риском аварий, утечек или использования радиоизотопов в качестве оружия [187].

В качестве замены радиоактивных источников можно использовать тормозное излучение, получаемое с помощью ускорителей заряженных частиц. Действительно, по сравнению с радиоизотопами ускорители являются более безопасным и намного более гибким источником рентгеновского излучения. Однако для того, чтобы стать адекватной заменой радиоизотопам, ускорители должны иметь сравнимые с ними вес, размеры и стоимость, сравнимые с последними. В настоящее время ускорители, используемые в качестве источника рентгеновского излучения, были разработаны в 1960-е годы, и с тех пор не претерпели значительных изменений [188].

До 80\% радиоизотопов, используемых в США, являются гамма-излучателями категории 2 по классификации МАГАТЭ: Иридий-192, Цезий-137, Кобальт-60 и Селен75 [189]. Энергия фотонов, излучаемых такими изотопами, варьируется от $265 \mathrm{keV}$ для Селена-75 до $1.25 \mathrm{MeV}$ для Кобальта-60 [190]. Из-за природы тормозного излучения, спектр которого в отличие от радиоизотопов сплошной и наиболее вероятное значение энергии меньше энергии электронов, ускоритель должен иметь 


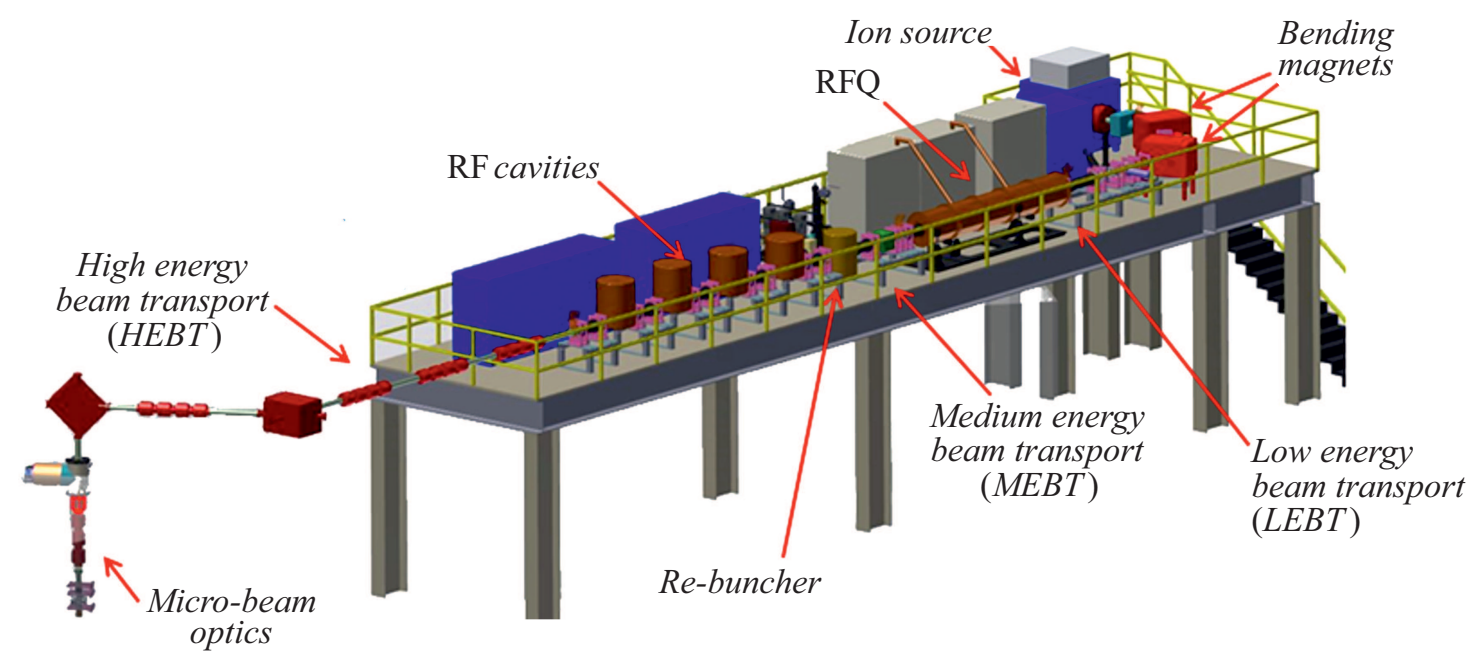

Рис. 9. Компактная установка для исследования быстропротекающих процессов при поражении материалов ядрами тяжелых элементов в реальном времени с помощью синхротронного излучения, основанная на ускорителе ионов с перестраиваемой энергией и типа частиц, предложенная для постройки в Аргоннской Национальной Лаборатории (ANL), CШA. Источник рисунка: Argonne Accelerator Institute (https://www.anl.gov/aai).
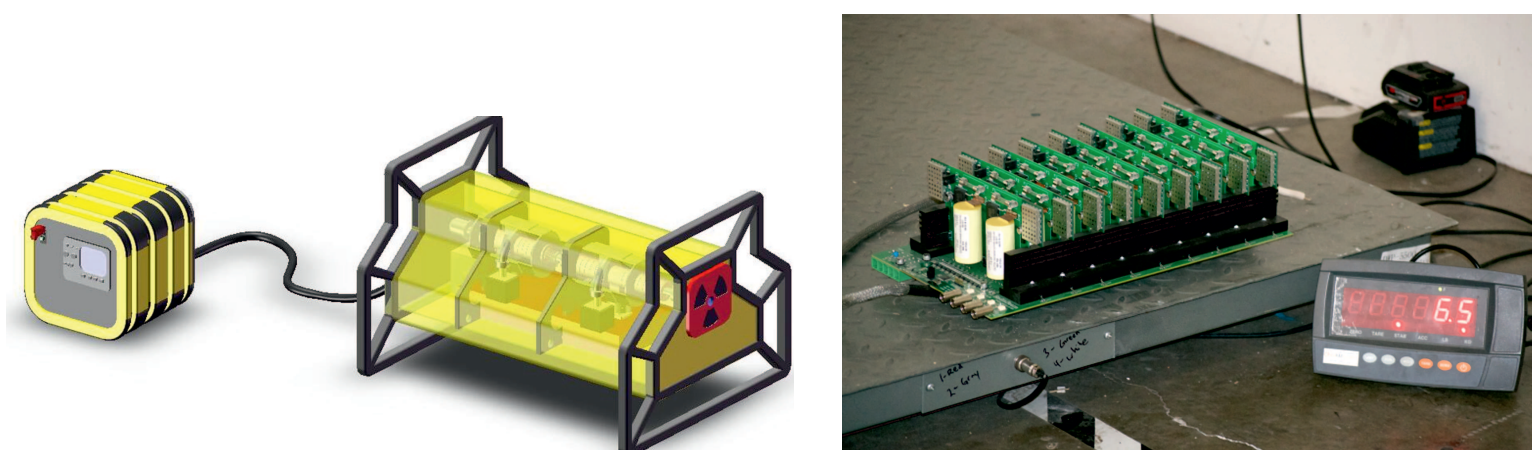

Рис. 10. Концепт переносного ускорителя для замены радиоактивных изотопов $(a)$ и блок модулятора Аркадьева-Маркса $(b)$, разработанный в RadiaBeam Technologies, США, позволяющий достичь напряжения $4.5 \mathrm{kV}$.

выходную энергию в 2-4 раза выше, чем энергия гаммаизлучения заменяемого радиоизотопа $[191,192]$.

Работы по созданию компактного ускорителя электронов (рис. 10,a) на такие энергии и мощности начались в Стэндфордской ускорительной лаборатории SLAC [193] и заключались в использовании структур $X$-частотного диапазона, питающихся от недорогих магнетронов с небольшой мощностью [194]. Однако предложенные размер и стоимость такого ускорителя все еще не позволяли использовать его в качестве адекватной замены радиоактивных изотопов.

Первым практическим шагом к созданию компактных ускорителей было использование медных структур с высоким шунтовым сопротивлением [195]. Такие ускорители были основаны на компенсированных бипериодических ускоряющих резонаторах [196] с внутренними или внешними ячейками связи [197]. Эти структуры имели значительную полосу пропускания, позволявшую использовать достаточное количество ячеек для дости- жения энергии $1 \mathrm{MeV}$ в односекционном ускорителе с питанием от источника мощностью менее $1 \mathrm{MW}$. К сожалению, производство ускоряющих структур с переменной фазовой скоростью [130] является сравнительно дорогим процессом, так как требует точного изготовления множества разных ячеек, их многоэтапную пайку и длительную настройку.

Важную роль в создании портативных ускорителей играет развитие технологи генераторов мощных импульсных сигналов, так называемых модуляторов, которые необходимы для питания импульсных СВЧгенераторов [198]. В частности, современные системы используют генераторы Аркадьева-Маркса [199] (рис. 10, $b$ ), которые не имеют громоздкого трансформатора, а состоят из массива мощных транзисторов с изолированным затвором (IGBT). Перспективные модуляторы на небольшие пиковые напряжения могут достигать размера и веса, допускающего переноса этого источника одним человеком. 

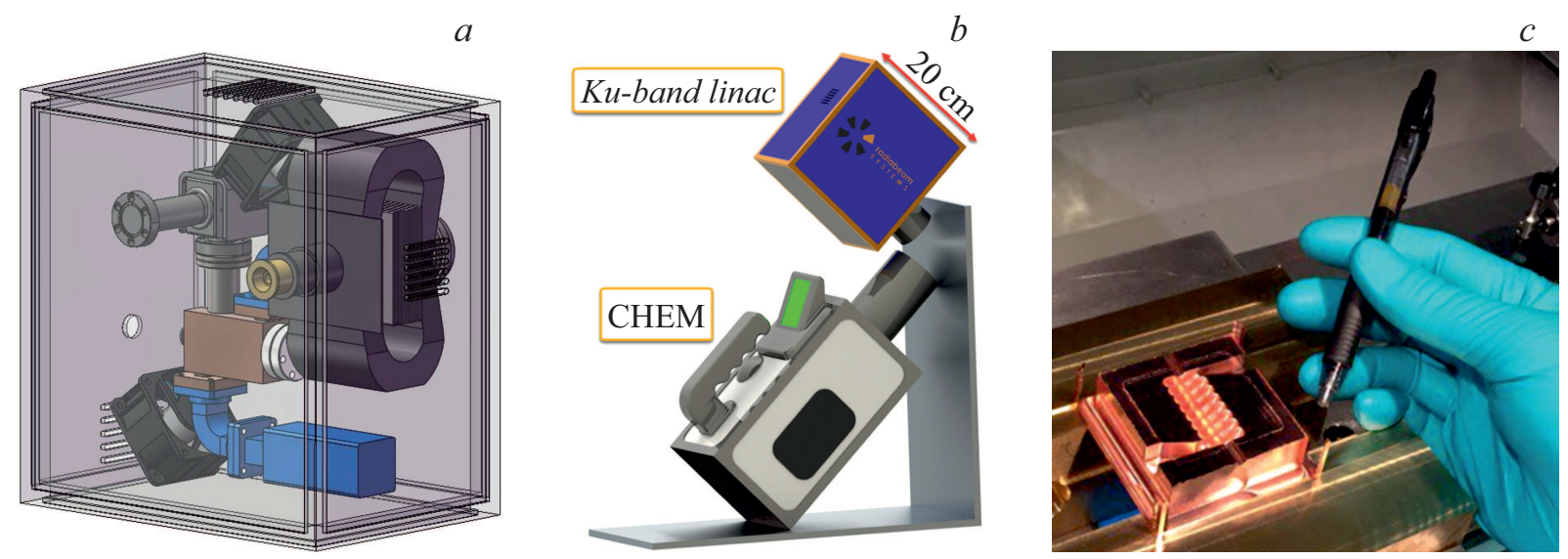

Рис. 11. Линейный ускоритель электронов Кu-частотного диапазона на энергию $180 \mathrm{keV}(a)$, разработанный RadiaBeam Technologies, США, для замены радиоизотопа кобальт-57 в мониторе обогащенного урана в коллекторных каскадах СНЕМ, используемых МАГАТЭ $(b) . c$ - прототип половинки разделенной структуры, выточенный из меди.

В настоящей главе будут рассмотрены три перспективных технологии для микроускорителей, предназначенных для замены радиоизотопов на примерах Кобальта-57 (122 keV), Иридия-192 $(380 \mathrm{keV})$, Цезия$137(0.66 \mathrm{MeV})$ и Кобальта-60 (1.25 MeV), на примере которых будет продемонстрировано использование современных подходов и технологий для достижения требований конкретных систем.

\subsection{1. Технология разделенных структур}

Радиоизотоп ${ }^{57} \mathrm{Co}$ излучает три линии гаммаизлучения с энергиями $14 \mathrm{keV}(9 \%), 122 \mathrm{keV}(86 \%)$ и $136 \mathrm{keV}(11 \%)$, имеет период полураспада 271.8 дней [200] и используется для калибровки аппаратуры, мессбауэровской спектроскопии, а также в составе радиофармпрепарата витамина $\mathrm{B}_{12}$ для изучения метаболизма организма и диагностики заболеваний, связанных с усвоением этого витамина [201].

Одним из примечательных применений этого изотопа является отслеживание наличия обогащенного урана на заводах по обогащению ядерного топлива с помощью газовых центрифуг [202] с использованием мониторов обогащенного урана в коллекторных каскадах (CHEM) [203]. Принцип действия усовершенствованной компактной модели СНЕМ на основе германиевого детектора [204] основан на следующем: осуществляется облучение трубы с потоком газа гексофлорида урана $\mathrm{UF}_{6}$ с помощью гамма-излучения изотопа кобальта- 57 с энергией выше $K$-края полосы поглощения, и проводится измерение сигналов пассивного излучения урана с энергией $185 \mathrm{keV}$ и индуцированного облучением излучения с энергией $98 \mathrm{keV}$. Таким образом, интенсивность сигнала индуцированного излучения пропорционально известной активности радиоизотопа, а интенсивность пассивного излучения пропорционально степени обогащения урана [205].
Программа развития МАГАТЭ подразумевает замену радиоизотопов в СНЕМ-мониторах на более безопасные технологии [206]. Для этого был разработан источник на основе линейного ускорителя заряженных частиц с энергией электронов до $180 \mathrm{keV}$, способный получать дозы излучения, сравнимые с активностью изотопа кобальта-57 в $1 \mathrm{mCi}$ [207] (рис. 11). Выбор энергии меньше $180 \mathrm{keV}$ обусловлен требованием к отсутствию маскировки сигнала пассивного излучения урана. Расчеты показали, что, несмотря на сплошной спектр тормозного излучения ускорителя, он может эффективно заменить монохроматический источник на основе радиоизотопа [82]. Примечательно, что с помощью применения новых технологий, таких, как малогабаритные мониторы $K u$-частотного диапазона, применяемые для воздушной диспетчеризации [208], а также разделенных ускоряющих структур, удалось достичь сверхкомпактных $(20 \times 20 \mathrm{~cm}, 5 \mathrm{~kg})$ размеров источника и невысокой стоимости за счет снижения количества и времени изготовляемых компонентов и их сборки, даже по сравнению с рентгеновскими трубками, традиционно применяемыми для этих энергий [209]. Подобная замена была бы невозможна с использованием существующих ускорителей, разработанных на основе традиционных технологий.

Данный дизайн позволяет масштабирование до энергий $\sim 1 \mathrm{MeV}$. Ограничение связано с отсутствием компактных магнетронов на мощности больше $250 \mathrm{~kW}$, а также сложностями с охлаждением компактных структур. Тем не менее сверхкомпактный ускоритель на такие энергии может служить заменой ряда радиоизотопов, например, селена-57, а также благодаря возможности добавления функции вариативности энергии использоваться для переносных систем радиографии, включая адаптивные методы радиографии, описанные в разд. 2.1.1.

Для более высокоэнергичных изотопов, таких, как один из самых распространенных радиоизотопов кобальт-60, применяющийся для стерилизации пищевых 
$a$

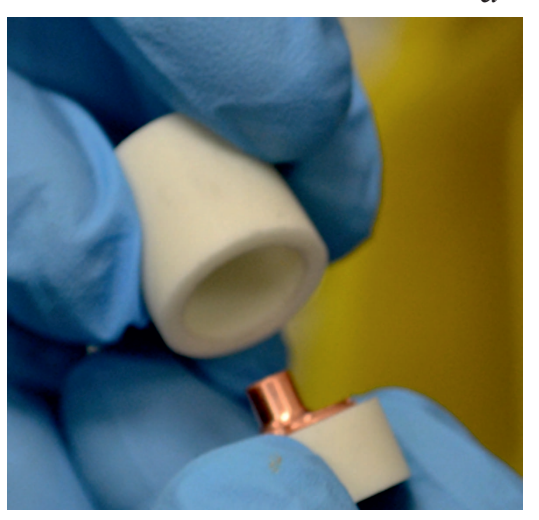

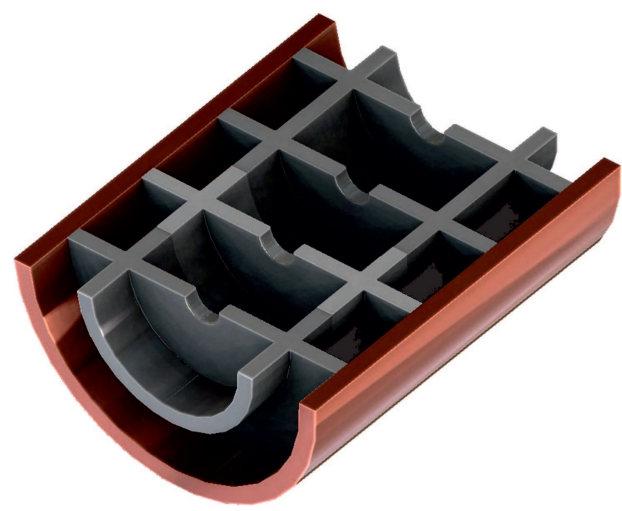

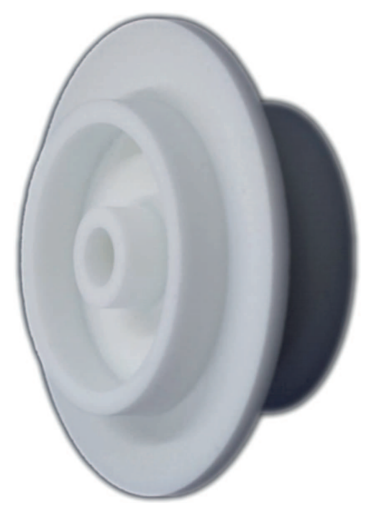

Рис. 12. Диэлектрические структуры для прикладных ускорителей: Ячейки гибридной метало-диэлектрической ускоряющей структуры (DLA) $X$-частотного диапазона, разработанной в RadiaBeam Technologies, CША $(a)$, и структуры со вспомогательным диэлектриком (DAA) $(b, c)$, предложенные Токийским Технологическим Университетом, Япония [362]. Ячейки крайне просты в изготовлении и затем вставляются в гладкую медную трубку. Источники фотографий: $a-$ A.B. Смирнов, RadiaBeam Technologies (https://radiabeam.com), $c-$ D. Satoh, Tokyo Institute of Technology (https://www.titech.ac.jp).

продуктов, медицинских инструментов и материалов, активации посевного материала, обеззараживания отходов, радиотерапии, дефектоскопии и радиоизотопных источниках энергии $[210,211]$, наиболее оптимальной является работа в $X$-частотном диапазоне, для которого существует большое разнообразие компактных мощных источников ВЧ-питания, в том числе и перспективных [212].

\subsection{2. Диэлектрические ускорители}

Изотоп иридия ${ }^{192} \operatorname{Ir}$ с периодом полураспада 73.83 дня является очень сильным гамма-излучателем с семью линиями в диапазоне от 200 до $600 \mathrm{keV}$ [213] и широко применяется в промышленной радиографии для обнаружения трещин в металлах [214]. Кроме того, этот изотоп применяется в радиотерапии, и, в частности, в брахитерапии [215]. Однако наибольшую известность этому радиоизотопу принесло его лидерство в списке наиболее часто пропадающих в США. Количество пропаж уже достигло уровня, достаточного для изготовления так называемой „грязной бомбы“ [216]. Для замены этого радиоизотопа требуется разработка компактного ускорителя электронов с выходной энергией $1-1.5 \mathrm{MeV}$ и дозой, эквивалентной $~ 200 \mathrm{Ci}$ [200].

Привлекательной альтернативой медным структурам для компактных ускорителей являются диэлектрические структуры, в которых гладкий металлический волновод частично заполнен диэлектриком (DLA) [217,218]. Действительно, длина волны в такой структуре будет пропорциональна $\varepsilon^{-1 / 2}$. Однако практическое применение ограничивается рядом проблем, среди которых можно отметить мультипакторный разряд [219] и накапливание заряда в диэлектрике, способное привести к разряду и повредить структуру [220]. В микроускорителях эти проблемы усугубляются значительными потерями частиц в процессе группировки из-за низкоэнергетичной инжекции (около $10-15 \mathrm{keV}$ ). Другая проблема заключается в широком диапазоне фазовых скоростей (от $\beta=0.3$ и ниже до $\beta=1.0$ ). Обеспечение низких фазовых скоростей требует применения диэлектриков с высокой диэлектрической проницаемостью $(\varepsilon>20)$, что увеличивает диэлектрические потери и снижает энергетическую эффективность [221].

Одним из способов решить проблемы гладких диэлектрических структур является использование гибридной метало-диэлектрической структуры, так называемая диэлектрическая структура с кольцами и диафрагмами (DAR) [222], являющаяся модификацией известной структуры с шайбами и диафрагмами [196,223,224]. Данная структура является квазипериодической и способна эффективно работать даже при низких значениях фазовой скорости $(\beta \sim 0.1-0.2)$. Ускоряющие ячейки в такой структуре состоят из металлических дисков с короткими трубками дрейфа, которые вставляются между диэлектрическими кольцами, что обеспечивает исключительную простоту изготовления [225] (рис. 12,a).

Другой альтернативой гладким диэлектрическим структурам является структура со вспомогательным диэлектриком (DAA) [226], в которой и кольцо, и диафрагма изготовлены из диэлектриков [227] (рис. 12, $b, c)$. Такая структура имеет огромные значения шунтового сопротивления, достигающие $650 \mathrm{M} \Omega / \mathrm{m}$ в $C$-частотном диапазоне, поскольку распределение электромагнитного поля ускоряющей волны может эффективно перераспределяться в диэлектрической части, тем самым снижая ВЧ-потери на металлических стенках структуры. Здесь стоит особо отметить значительные успехи, достигнутые в разработке и получения диэлектриков со сверхнизкими значениями тангенсов диэлектрических потерь, таких как оксид магния $\mathrm{MgO}\left(\operatorname{tg} \delta=6.0 \cdot 10^{-6}\right.$ [228]), оксид алюминия $\mathrm{Al}_{2} \mathrm{O}_{3}\left(\operatorname{tg} \delta=1.2 \cdot 10^{-5}\right.$ [229]), а также кера- 
миках на основе сложных оксидов танталата и вольфрамата бария-магния $\mathrm{Ba}\left(\mathrm{Mg}_{1 / 3} \mathrm{Ta}_{2 / 3}\right) \mathrm{O}_{3}-\mathrm{Ba}\left(\mathrm{Mg}_{1 / 2} \mathrm{~W}_{1 / 2}\right) \mathrm{O}_{3}$ $\left(\mathrm{BMT}-\mathrm{BMW}, \operatorname{tg} \delta=2.5 \cdot 10^{-5}[230]\right)$.

\subsection{3. Структуры со встроенным источником питания}

Еще одним примечательным радиоизотопом является цезий-137 с периодом полураспада около 30.17 лет [231], который через цепочку распадов излучает фотоны с энергией $661.7 \mathrm{keV}$. Этот источник используется в радиотерапии, измерительной технике, для радиационной стерилизации пищевых продуктов, медицинских препаратов и лекарств [232], в радиоизотопных плотномерах и уровнемерах [233], а также для интегрального гаммакаротажа нефтяных скважин [234].

Процесс каротажа заключается в помещении источника излучения в скважину для определения свойств геологических формирований, окружающих скважину. Данные, полученные во время каротажа, помогают определить литологическую классификацию пород [235], структурные особенности формирований, такие, как состав камней (известняк, сланец, песчаник и т.д.), их свойства (пористость, проницаемость, наличие жидкости и т.д.) и целостность (наличие структурных дефектов, угрозы проседания), а также информацию о размерах и форме самой скважины, наличие в ней жидкости и ее свойства [236]. Каротаж, осуществляемый непосредственно на месте добычи, требует проведения непрерывных измерений даже в то время, когда температура и давление в скважине имеют рабочие значение. В случае замены радиоизотопов ускорителями заряженных частиц, последние должны свободно передвигаться в скважине диаметром около 9-10 cm, работать в диапазоне температур до $150^{\circ} \mathrm{C}$ на глубине около $1 \mathrm{~km}$, а также в условиях вибраций и ускорений до $2 \mathrm{~g}$ [237]. Традиционные и даже компактные схемы ускорителей на основе магнетронов не способны работать в таком диапазоне условий из-за габаритов, а также нестабильности частоты генератора, ферритовых компонентов и ускоряющей структуры.

Для устранения этой проблемы был предложен ряд решений, совмещающих источник ВЧ-питания (клистрон) и ускоряющую структуру в едином вакуумном интерфейсе с общим пучком [238-242]. В такой структуре сильноточный пучок сначала передает свою энергию для возбуждения СВЧ в ускоряющей структуре, а затем небольшая его часть продолжает ускорение в этом возбужденном поле. Таким образом, обеспечивается необходимая синхронность частоты источника и ускоряющей структуры [243].

Для стабильной работы в широком диапазоне температур, ускоряющая структура должна обладать значительной широкополосностью [244]. Хорошим примером такой структуры, в которой также реализован принцип простоты и эффективности, является встречно-штыревая ускоряющая структура, состоящая из чередующихся пар штырей, повернутых на $90 \mathrm{deg}$ относительно друг друга [245] (рис. 13). Она позволяет реализовать широкий диапазон фазовых скоростей $(\beta<0.3)$, большую широкополосность (до $37 \mathrm{MHz}$ частотного разделения в 37-ячееч|-ной структуре) и сравнительно высокое шунтовое сопротивление.

\section{3. Перспективные медицинские ускорители}

Рак является глобальной проблемой, причиной до 13\% смертей по всему миру, а также одной из наиболее быстро распространяющихся болезней на земле [246]. Радиотерапия опухолей с помощью пучков частиц, применяемая в качестве первичного средства лечения или совместно с химиотерапией, хирургией или другими методами, используется для более $60 \%$ онкобольных, и имеет отношение к практическе половине случаев излечения [247].

Три наиболее популярных технологии для радиоонкологии используют пучки фотонов, электронов и адронов (протонов и тяжелых ионов) [248]. В настоящее время фотонная радиотерапия является наиболее зрелой и продвинутой технологией, и продолжает развиваться, повышая свою медицинскую эффективность [249]. Тем не менее существуют причины полагать, что в ближайшем будущем адронная радиотерапия может обогнать по эффективности фотонную терапию для отдельных случаев, таких, как глазные меланомы, хордомы, опухоли ЦНС у детей и др. [250]. Терапевтическая эффективность данного метода обусловлена эффектом Брэгга, когда протоны и тяжелые частицы высвобождают большую часть своей энергии в конце траектории [251], что позволяет минимизировать повреждение здоровых тканей, окружающих опухоль. Однако несмотря на это, адронная терапия используется только в $1 \%$ лечения онкобольных [252], что в большей степени обусловлено высокой стоимостью таких систем. Например, стоимость центра протонной терапии на основе циклотрона или синхротрона может достигать 100 миллионов долларов США и выше [253], в то время как традиционные фотонные системы стоят около 3 миллионов долларов США [254].

Несмотря на свое широкое использование в развитых странах, радиотерапия остается малодоступной в странах с низким и средним уровнем доходов [255], где распространенность технологий для терапии на душу населения может быть на 2 и более порядка ниже, чем в США [256]. И хотя в таких странах проживает до $85 \%$ населения земного шара, в доступе имеется всего около 4.5 тысяч систем для радиотерапии, что как минимум вдвое меньше, чем требуется для эффективной работы системы здравоохранения [257]. Более того, источники, используемые в развивающихся странах, используют устаревшие технологии ускорителей или радиоизотопы.

Таким образом, современные тенденции развития ускорительных технологий для радиотерапии направлены на повышение доступности и снижение стоимости си- 
$a$

$b$
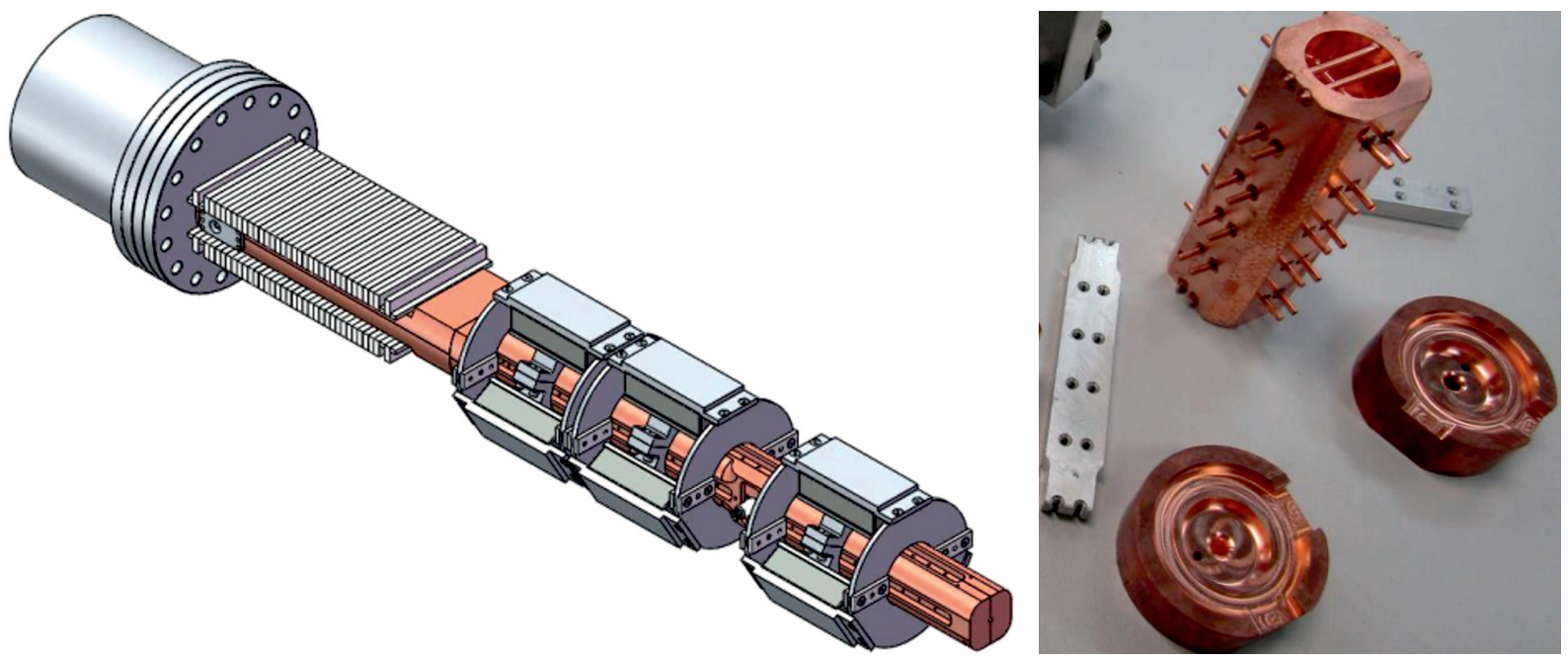

Рис. 13. Модель компактного ускорителя для каротажа нефтяных скважин на энергию $1 \mathrm{MeV}$ со встроенным источником питания $(a)$ на основе штыревой ускоряющей структуры $X$-частотного диапазона $(b)$, разработанный в RadiaBeam Technologies, США [363]. Рисунки А.В. Смирнова, RadiaBeam Technologies (https://radiabeam.com).
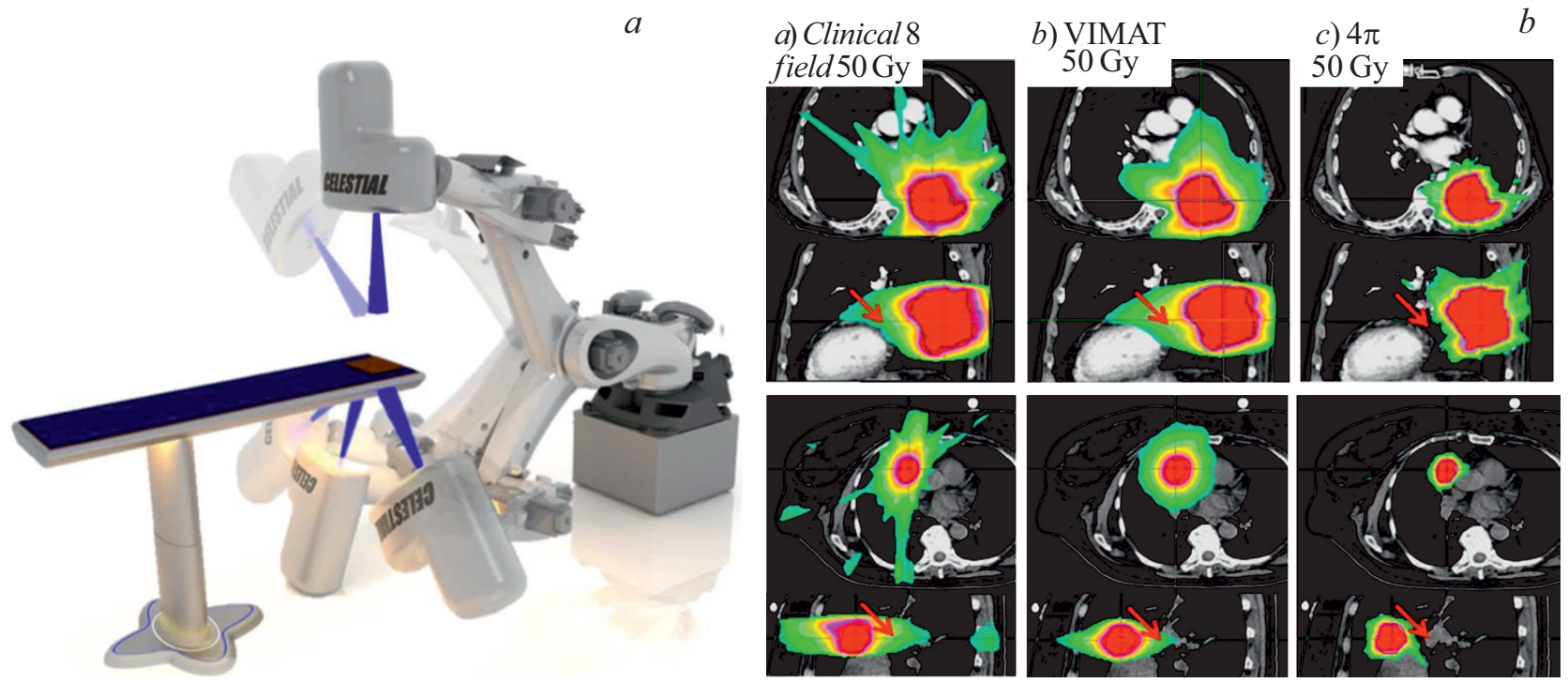

Рис. 14. Модель установки для 4л-терапии (a), разработанная Калифорнийским Университетом в Лос Aнжелесе (UCLA), совместно с RadiaBeam Technologies, США, и сравнение распределения доз облучения вокруг опухолей с традиционными средствами радиотерапии (b) [364]. Источник рисунков: University of California Los Angeles (https://www.uclahealth.org)

стем, повышение биологической эффективности лечения и уменьшения побочных эффектов, а также на развитие новых методов лечения, таких как, например, терапия движущихся опухолей, позволяющих распространить радиотерапию на большее количество типов рака. Эти тенденции хорошо согласуются с общими тенденциями развития ускорительной техники прикладного назначения: компактность, вариативность и экономическая эффективность.

В следующем разделе будет рассмотрено несколько перспективных систем для радиотерапии, для реали- зации которых требуется разработка и использование новых ускорителей.

\subsection{1. Ускорители для фотонной терапии}

Как упоминалось выше, одной из проблем современной фотонной радиотерапии является неравномерность распределения дозы облучения и повреждение здоровых тканей вокруг опухоли. Эта проблема может быть решена облучением пациента с большого числа разных некомпланарных направлений, путем, например, вращения источника излучения вокруг пациента в пре- 

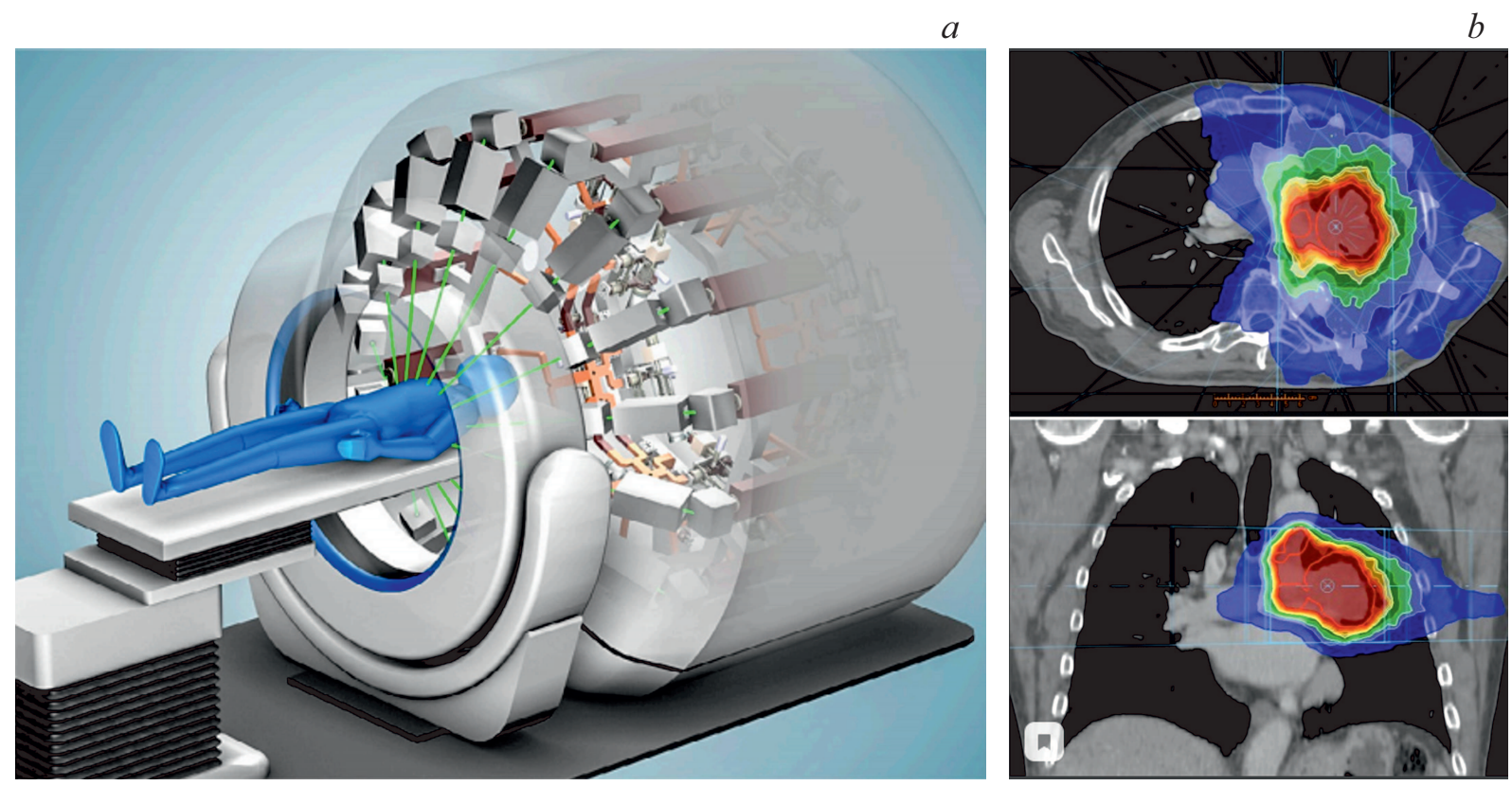

Рис. 15. Модель установки для FLASH-терапии (a), разработанная Стэнфордским Университетом и Университетом Индианы, США [365], и распределение дозы облучения вокруг опухоли легких в такой системе $(b)$ [366]. Источники рисунков Tibaray Inc. (https://tibaray.com/).

делах полного телесного угла $4 \pi$ [258]. Такой метод получил название $4 \pi$-терапии [259] (рис. 14). Данная технология позволяет существенно увеличить эффективность доставки дозы в опухоль, не повреждая окружающие ткани. Ученые Калифорнийского Университета в Лос-Анджелесе (UCLA) показали, что с помощью $4 \pi$ терапии можно улучшить местный контроль рецидивных опухолей головы и шеи с $35 \%$ до более $90 \%$ [260], а центрально расположенных опухолей легкого - практически до $100 \%$ [261].

Реализация метода $4 \pi$-терапии напрямую зависит от источника фотонов, т. е. ускорителя заряженных частиц. В традиционных системах радиотерапии, таких, как Carm [262] или CyberKnife [263], вращающихся вокруг пациента, эту технологию реализовать практически невозможно из-за габаритов ускорителей [264]. Однако разработка компактных ускорителей $X$-частотного диапазона, и использование разделенных ускоряющих структур с высоким шунтовым сопротивлением, позволяющим применять недорогие модели магнетронов, одновременно с использованием роботизированной системы доставки и коллиматоров переменной формы, могут позволить сделать $4 \pi$-терапию доступной уже в ближайшее время [265].

Другой проблемой в радиотерапии является ошибка позиционирования пучка фотонов в пространстве из-за движения пациента во время процедуры облучения [266]. Традиционные подходы к решению этой проблемы основаны на парадигме, что доставка дозы облучения осуществляется намного медленнее времени срабатывания нервных импульсов, приводящих пациента в движе- ние [267]. Однако последние доклинические испытания показали, что при сверхбыстрой доставке больших доз (> $50 \mathrm{~Gy} / \mathrm{s})$ в опухоль значительно уменьшается повреждение окружающих тканей и увеличивается эффективность лечения [268]. Такой метод облучения получил название FLASH-терапии (рис. 15).

Для реализации задачи сверхбыстрой доставки дозы облучения в рамках компактной и экономически эффективной платформы необходима разработка либо мощного ускорителя с магнитной системой вращения пучка, либо массива из линейных ускорителей, расположенных вокруг пациента. Стоимость такой системы будет в большой степени определяться стоимостью отдельных ускорителей и систем ВЧ питания. Решение этой проблемы, предложенное Стэнфордской Ускорительной Лабораторией SLAC, включает использование ускоряющих структур $X$-частотного диапазона с индивидуальным питанием ячеек, что позволило увеличить шунтовое сопротивление такой структуры до 4 раз по сравнению с традиционными медицинскими ускорителями $S$-частотного диапазона [269], и это позволяет значительно сократить стоимость системы за счет меньшего количества СВЧ генераторов. Кроме того, применение технологии разделенной структуры позволило уменьшить и стоимость изготовления самого ускорителя.

Интересно также отметить, что FLASH-терапия не ограничивается использованием фотонов [270]. Среди лабораторий, работающих над этой проблемой, Институт Кюри, Франция, разработал систему протонного облучения по технологии FLASH для мелких животных [271]. Кроме того, недавно Университетский Меди- 
цинский Центр Гронинген, Нидерланды, усовершенствовал одну из двух протонных установок для доставки доз со скоростью более $33 \mathrm{~Gy} / \mathrm{s}$ [272]. О миниатюризации адронных ускорителей будет подробно рассказано в следующей главе.

\subsection{2. Ускорители для адронной терапии}

Как уже упоминалась выше, терапия с помощью тяжелых частиц, адронов имеет преимущество в виде эффекта Брэгга, позволяющее пучку проходить сквозь вещество, практически не взаимодействуя с ним, и доставлять радиационную дозу непосредственно в опухоль. Несмотря на то, что для адронной терапии существует много кандидатов, таких как ионы гелия [273] или кислорода [274], в настоящее время для лечения опухолей используется только два типа частиц: протоны и ионы углерода [275]. Несмотря на большую стоимость и сложность углеродных ускорителей, связанную с меньшим отношением заряда к массе для ионов углерода, а следовательно, и большие затраты энергии и меньший темп ускорения тяжелых частиц, углеродная терапия имеет потенциал излечения таких радиорезистентных опухолей, как неоперабельная саркома кости и мягких тканей [276], забрюшинные саркомы, фиброзная гистиоцитома, липосаркома, опухоли периферического нерва влагалища [277], остеосаркома грудины [278] и другие [279], ранее считавшиеся неизлечимыми [280].

В настоящее время для адронной терапии используется два типа ускорителей: циклотроны для ускорения протонов и синхротроны для ускорения протонов и ионов [281]. Помимо стоимости в сотни миллионов долларов США [253], эти ускорители имеют ряд существенных недостатков. Циклотроны имеют фиксированную энергию пучка, что не позволяет эффективно изменять глубину его проникновения в тело пациента [282]. Синхротроны, хотя и имеют такую возможность, но каждое изменение энергии занимает около $1 \mathrm{~s}$ [283], что приводит к длительному времени лечения опухолей и ограничивает общую пропускную способность установки. Другим обстоятельством, препятствующим распространению адронной терапии в ее нынешнем состоянии является ее неприспособленность к лечению движущихся опухолей, что также обусловлено крайне низкой скоростью перестройки энергии. Конкурентоспособные системы должны обладать скоростью вариативности энергии достаточно высокой для того, чтобы синхронизовано работать с системой сканирования опухоли $(<20 \mathrm{~ms})[63]$.

Перспективными технологиями для адронной терапии представляются линейные ускорители, имеющие большую вариативность энергии [284] и способные изменять ее с частотой следования импульсов $(\sim 1 \mathrm{~ms})$. Оптимальным решением такой задачи является ускоритель, в последней секции которого амплитуда и фаза ВЧ мощности управляется электронно. Однако линейные ускорители на необходимые энергии $250 \mathrm{MeV}$ для протонов и $450 \mathrm{MeV} /$ nucleon для ионов углерода [285], построенные по традиционным схемам и работающие на частотах метрового диапазона, имеют размеры, делающие их непригодными для использования в адронотерапии. Например, Linac4 для ЦЕРНа, ускоряющий протоны до $160 \mathrm{MeV}$, имеет длину 86 метров [286].

Для миниатюризации адронных ускорителей был разработан ряд инновационных технологий, которые были в полной мере реализованы в протонном ускорителе для онкотерапии TULIP (ЦЕРH, Швейцария) [287]. Учеными ЦЕРНа и Национальной лаборатории INFN во Фраскати, Италия, была показана возможность ускорения протонов в структурах, работающих в 10-сантиметровом $(S$ - $)$ диапазоне [288], что позволило уменьшить размеры высокоэнергетичных секций до 10 раз. Затем ученым ЦЕРНа удалось увеличить частоту начальной секции ускорителя типа RFQ до $750 \mathrm{MHz}$ [289], что позволило уменьшить поперечные размеры этой структуры до $13.4 \mathrm{~cm}$, а вес до $220 \mathrm{~kg}$ [290]. Наконец, разработка ускоряющих структур для протонов с малой фазовой скоростью $\beta=0.38$ и имеющих градиенты $50-80 \mathrm{MeV} / \mathrm{m}[291,292]$ позволила уменьшить размер всего ускорителя до $\sim 20 \mathrm{~m}$ и разместить его на вращающейся вокруг пациента платформе [293]. Главным преимуществом такой схемы станет отсутствие в нем магнитного портала для вращения пучка (гэнтри), вес и стоимость которой вносили бы значительную часть в общую стоимость и габариты системы [294] (рис. 16, 17, a).

Похожие тенденции наблюдаются и в разработке ускорителей для углеродной терапии [295]. Из-за максимального отношения заряда ионов углерода ${ }^{12} \mathrm{C}^{6+} \mathrm{q} / \mathrm{A}=1 / 2$, что снижает темп их ускорения, по сравнению с протонами и что в совокупности с необходимостью ускорять эти ионы до более высоких энергий, приведет к чрезмерно большим габаритам таких ускорителей. Например, линейная часть гибридного (циклотрон + линак) ускорителя САВОТО, работающая в диапазоне энергий $150-410 \mathrm{MeV} /$ nucleon в $C$-частотном диапазоне $(5.712 \mathrm{GHz})$, имеет длину $24.1 \mathrm{~m} \mathrm{[296].}$

Примером полностью линейного ускорителя ионов углерода до энергии $450 \mathrm{MeV} /$ nucleon может служить проект ACCIL [297], разработанный учеными Аргоннской Национальной Лаборатории (ANL) совместно с RadiaBeam Technologies, США. В данном ускорителе благодаря использованию ускоряющих структур, работающих на первой отрицательной гармонике ускоряющей волны [298-300] (рис. 17, $b$ ), удалось снизить энергию, c которой возможно применение высокоградиентных структур $S$-частотного диапазона до $45 \mathrm{MeV} /$ nucleon $(\beta=0.3)$, что позволило сократить общую длину до $40 \mathrm{~m}$ [301], сделав ее сравнимой с циклическими ускорителями. Помимо этого, пучок ускорителя ACCIL синхронизован с системой сканирования пучка на основе томографии, позволяющей определять текущее положение опухоли и одновременно производить лечение [302]. Благодаря возможности изменять 

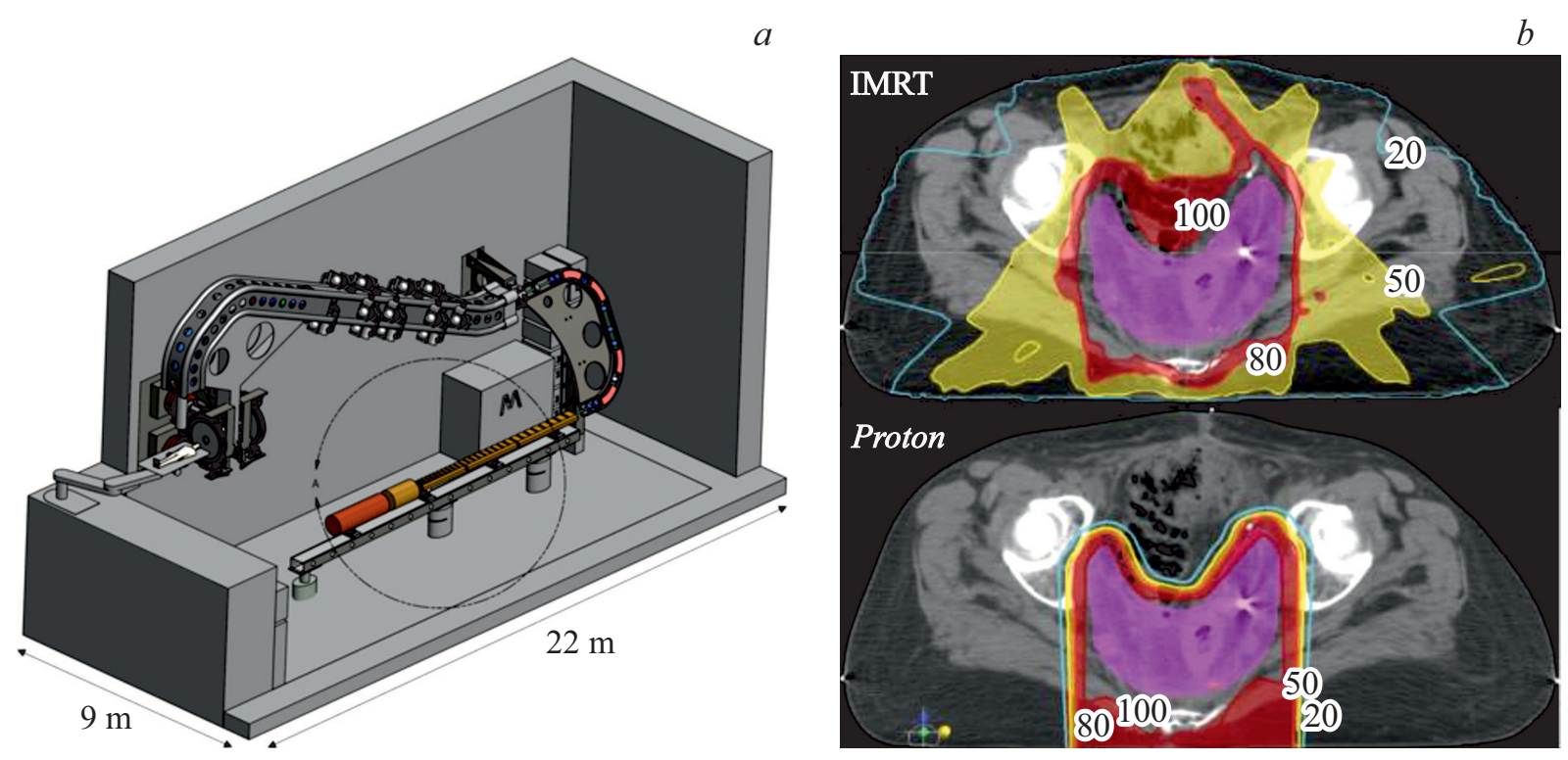

Рис. 16. Модель компактного линейного ускорителя для протонной терапии TULIP, способного вращаться вокруг пациента (a) [287], разработанного Европейским центром по ядерным исследованиям (CERN), Швейцария, и не требующая гэнтри. На рисунке $(b)$ показано сравнение распределения дозы около опухоли при протонной терапии (снизу) и традиционной IMRT (сверху). Источники рисунков: $a$ - Centre Européenne pour la Recherche Nucléaire (https://cern.ch), $b$ - Advanced Oncotherapy (https://www.advancedoncotherapy.com).

$a$

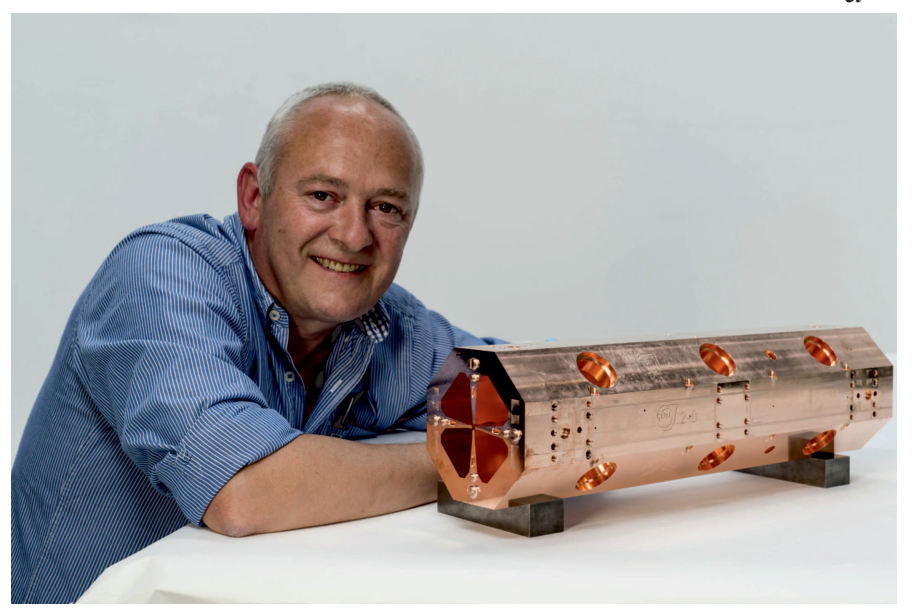

$b$

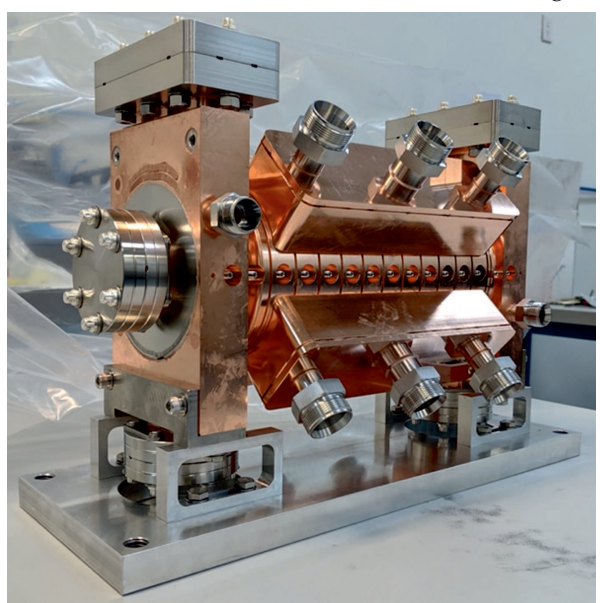

Рис. 17. Перспективные технологии для компактных ускорителей для адронной терапии: $a-$ компактная ускоряющая секция $\mathrm{RFQ}$, работающая на частоте $750 \mathrm{MHz}$ для ускорения протонов до энергии $2.5 \mathrm{MeV}$ [367], разработанная Европейским центром по ядерным исследованиям (CERN), Швейцария, и секция высокоградиентной ускоряющей структуры для малых энергий частицы $(\beta=0.3)$, работающая на первой отрицательной гармонике электромагнитной волны $(b)$, разработанная RadiaBeam Technologies, США. Источники фотографий: $a-$ Centre Européenne pour la Recherche Nucléaire (https://cern.ch), $b$ - RadiaBeam Technologies (https://radiabeam.com).

энергию пучка от импульса к импульсу, этот ускоритель может прецизионно лечить даже движущиеся опухоли с помощью послойного облучения сродни принципу печати, используемому в $3 D$-принтерах [63]. Высокая частота повторения импульсов (до 1000 в s) позволит производить лечение всей опухоли в течение $5 \mathrm{~min}[65]$.
В заключение данной темы стоит упомянуть о лазерных технологиях для ускорения протонов. Принцип действия таких систем основан на взаимодействии лазера с твердой мишенью, что позволяет получить локальные статические поля с градиентом до $1 \mathrm{TV} / \mathrm{m}$, что делает их перспективными для использования в качестве ускорителя протонов для терапии [303]. Однако прогресс в 
этой области, достигнутый за последние 10 лет, заметно отстает как по результатам, так и по темпу развития ВЧускорителей [304]. Значения энергии, достигнутые в лазерных ускорителях, не превышают 60-80 MeV/nucleon как для протонов [305], так и для ионов углерода [306]. Ускорение протонов до $160 \mathrm{MeV}$ было продемонстрировано в Национальной Лаборатории Ливермора (LLNL), США с помощью огромных размеров лазера [307], но даже эти значения полученной энергии меньше необходимого для протонов уровня $250 \mathrm{MeV}$ и не дают возможности коммерческого использования таких ускорителей. Другими недостатками являются низкая интенсивность пучков, более чем на 3 порядка ниже требуемой [305], и значительная ширина энергетического спектра, достигающие 100\% для высоких энергий [308], что также нивелирует преимущества адронной терапии. Наконец, чрезмерные габариты лазера определяют минимальные размеры такой системы терапии, сравнимые с размером комнаты, а ее стоимость составляет 20 миллионов долларов США [309], что сравнимо по порядку с системами на основе ВЧ-ускорителей [310]. Приведенные факты дают основание полагать, что данные технологии могут получить должное развитие только в перспективе нескольких десятилетий.

\section{4. Перспективное применение сверхпроводящих ускорителей}

Сверхпроводящие ускорители являются стандартным инструментом в научных лабораториях [311] благодаря низким ВЧ-потерям в структуре по сравнению с нормальнопроводящими ускорителями [312]. Разумеется, достижение сверхпроводимости обусловлено значительными энергозатратами в гелиевых рефрижератоpax [313], но в целом энергетическая эффективность таких ускорителей выше в тех случаях, когда требуется получение непрерывных интенсивных пучков [314].

Существует ряд прикладных задач, в которых применение сверхпроводящих ускорителей было более выгодным и многообещающим по сравнению с нормальнопроводящими ускорителями, если сделать их более компактными (не более 5-6 m в длину), достичь простоты эксплуатации, автономности и возможности транспортировки, а также удовлетворительной энергетической эффективности и снизить стоимость до порядка 1 миллиона долларов США стоимость в пределах 1 миллиона долларов США. Кроме того, непрерывный режим работы, естественный для сверхпроводящих ускорителей, является более предпочтительным, чем импульсный, в котором работает большинство нормальнопроводящих ускорителей, для многих промышленных и научных применений. Например, в современных методах инспекции, описанных в разд. 2, непрерывный режим необходим для корректной работы детекторов.

Компактные непрерывные интенсивные $(10 \mathrm{~kW}-1 \mathrm{MW})$ пучки электронов или фотонов на энергии до $10 \mathrm{MeV}$ можно использовать для следующих задач [315]:

- для стерилизации продуктов питания, медицинских инструментов или воды [316];

- в энергетике для облучения биотоплива с целью улучшения его свойств [317], сжижения газовых продуктов в месте добычи [318] с целью повышения доли извлеченного природного газа, а также для облучения отработанного газа с целью удаления из него оксидов серы и азота [319];

- для вулканизации полимеров, в том числе и непосредственно на месте их нахождения. Например, можно вулканизировать полимеры защитных материалов для битума (или использовать их вместо битума) в асфальтированных дорогах для продления их срока службы [320];

- для систем инспекции грузов, основанных на методах непрерывного излучения (см. разд. 2.1.2).

Если вести речь о пучках электронов мощностью сотни киловатт при энергии до $10 \mathrm{MeV}$, то такие пучки могут ускоряться в относительно простых и дешевых нормальнопроводящих ускоряющих структурах без риска потери сверхпроводимости за счет гало пучка, при этом электронный КПД благодаря большому току пучка может превышать $80 \%$, если длина ускорителя приемлема для практических целей [321]. В случае, когда необходимы небольшие габариты системы (например, для выездной радиографии) или мощность порядка MW (например, для обеззараживания вод), применение сверхпроводящих ускорителей является более предпочтительным.

Сверхпроводящие ускорители также могут эффективно использоваться для ускорения тяжелых частиц (адронов). Например, для выскоэнергетичной имплантации ионов с энергий до $10 \mathrm{MeV}$ для производства подложек с глубокозалегающими слоями полупроводников или изоляторов [322], или производства радиоизотопов. Кроме того, такой ускоритель можно использовать в научных лабораториях с отсутствием системы распределения жидкого гелия.

\subsection{1. Автономные сверхпроводящие ускорители}

Несмотря на обилие прикладных задач, которые можно было бы эффективно решить с помощью сверхпроводящих ускорителей (рис. 18), их коммерческое использование в настоящее время ограничено из-за сложности и стоимости таких систем. Традиционные сверхпроводящие ускорители требуют наличия центральной криогенной системы для получения и распределения жидкого гелия, или же его постоянную доставку. Кроме того, содержание и обслуживание этих систем требуют особой квалификации и внимания персонала, поскольку при такой нештатной ситуации как квенч [323], размораживание или механическое повреждение, сверхпроводящий ускоритель или его часть может навсегда выйти из строя [324,325]. 


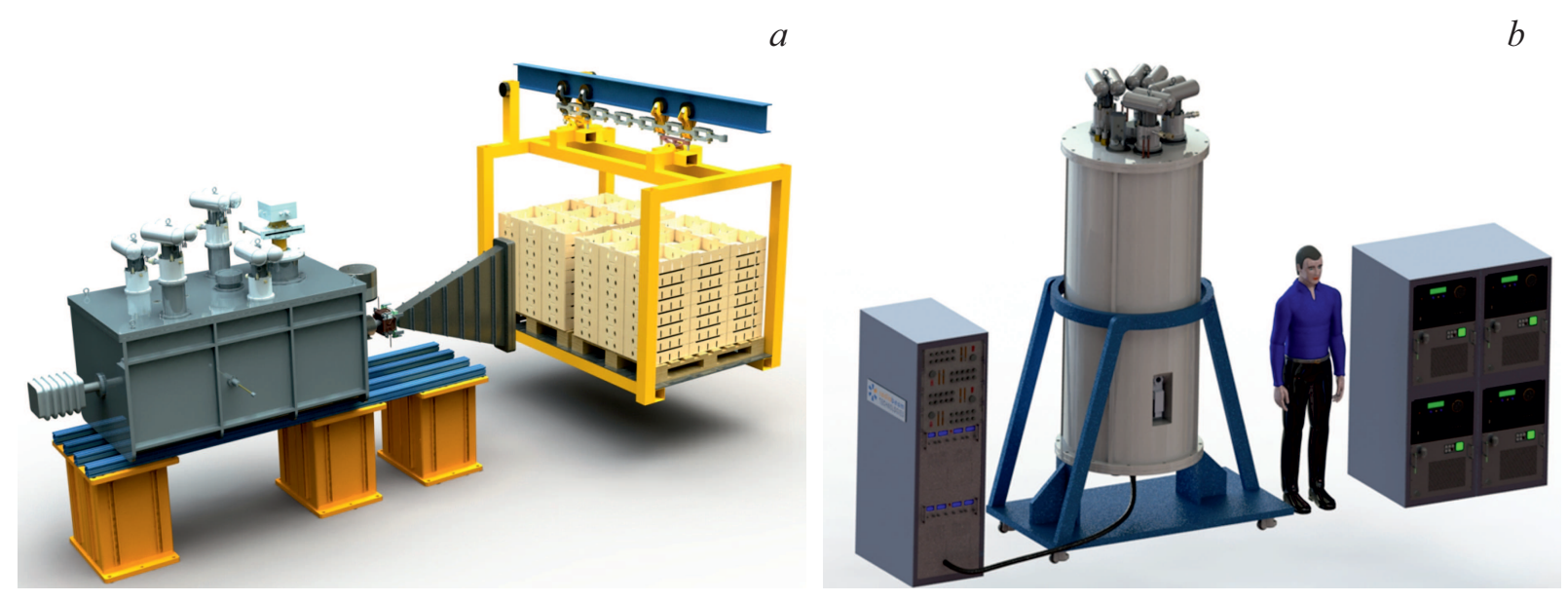

Рис. 18. Модели автономных сверхпроводящих ускорителей электронов $(a)$ и ионов $(b)$ на основе коммерческих криокулеров, разработанные RadiaBeam Technologies, CША, совместно с Национальной Ускорительной Лабораторией им. Э. Ферми (FNAL), США и Аргоннской Национальной Лабораторией (ANL), США.

$a$
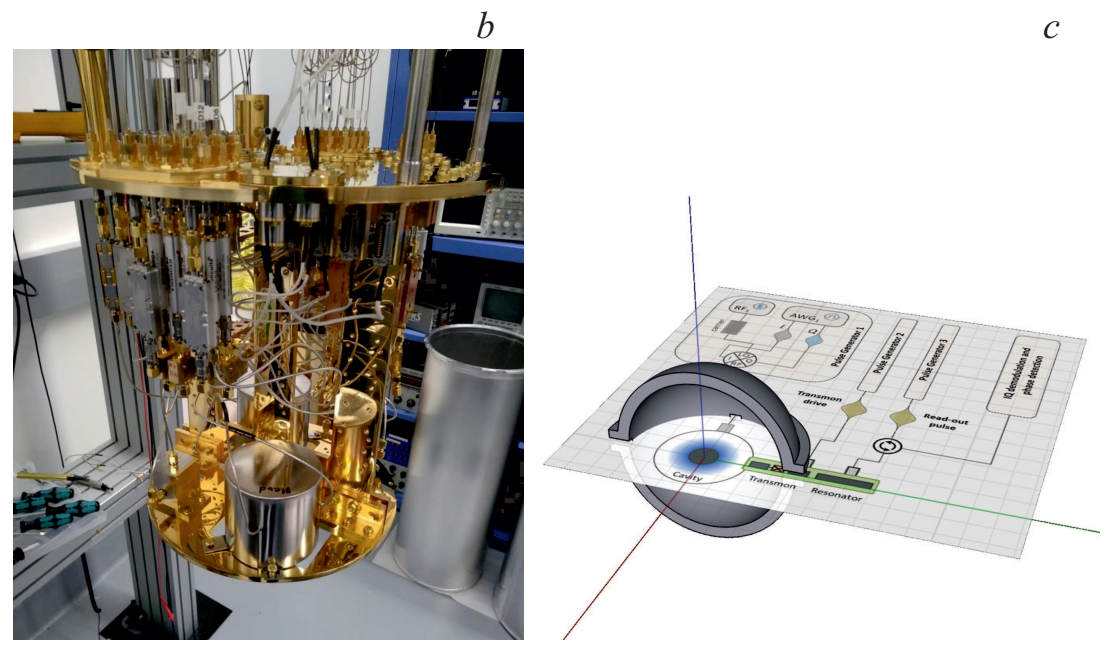

Рис. 19. Сверхпроводящий четвертьволновый резонатор для квантовой памяти квантового компьютера $(a)$, разработанный RadiaBeam Technologies, США, и изготовленный методом трехмерной печати из сплава титана Ті64. Ниобиевые резонаторы такого типа были протестированы в рефрижераторе растворения Университета Чикаго, США $(b)$. На рисунке $(c)$ изображена схематика квантового бита на основе сверхпроводящего резонатора.

Развитие технологий в области сверхпроводимости, произошедшее за последнее десятилетие, позволяют значительно упростить дизайн сверхпроводящих ускорителей, а также сделать их мобильными, энергетически и экономически эффективными при мощности пучка порядка $1 \mathrm{MW}$ [326], чего невозможно достичь с помощью нормальнопроводящих установок. Из таких технологий можно выделить следующие: усовершенствование формы резонаторов [327], процесса их производства [328] и обработки поверхности, в частности, инфузии атомами азота [329], а также применения техник быстрого охлаждения для избежания так называемой $Q$-болезни [330,331], позволили достичь значений собственных добротностей до $10^{11}$ [332] при работе на ускоряющих градиентах до $20 \mathrm{MV} / \mathrm{m}$ [333,334].
Другим прорывом в области сверхпроводников стало усовершенствование техники напыления станнида триниобия $\mathrm{Nb}_{3} \mathrm{Sn}$ на резонаторы сложных форм и доведения ее до уровня отлаженных технологий [335]. Этот материал примечателен тем, что имеет критическую температуру $18.5 \mathrm{~K}$, что почти вдвое выше, чем у ниобия, а также более высокое критическое магнитное поле [336]. Благодаря этим свойствам резонаторы, имеющие покрытие из $\mathrm{Nb}_{3} \mathrm{Sn}$, обладают меньшими потерями СВЧмощности, а значит, требуют рефрижераторов меньшего размера [337]. Одним из практических аспектов применения этого материала является то, что такие резонаторы могут работать при температуре $4 \mathrm{~K}$, что существенно увеличивает энергетическую эффективность рефрижератора [338], но имеют потери, сравнимые с 
потерями в ниобиевых резонаторах, работающих при температуре $2 \mathrm{~K}$.

Уменьшение ВЧ-потерь в резонаторах до значений $<10 \mathrm{~W}$, наряду с появлением автономных криорефрижераторов (криокулеров) с мощностью > 2W [339], позволило начать разработку автономных ускорителей электронов и ионов, не требующих подключения к центральному рефрижератору или постоянного снабжения жидким гелием [340-342]. Более того, разработка эффективной системы контактного охлаждения резонаторов [106] сделала такие системы безжидкостными, что существенно упрощает их практическое использование [343].

\subsection{2. Использование ускорительных технологий для квантовых компьютеров}

В заключение данного обзора необходимо упомянуть одно из наиболее перспективных применений технологий высокочастотной сверхпроводимости, а именно для создания элементов квантовой памяти на основе перехода Джозефсона [344]. Основным элементом квантового компьютера является квантовый бит или кьюбит, работающий на принципах суперпозиции [345] и запутывания [346]. Из-за взаимодействия кьюбита с окружающей средой его квантовое состояние может потеряться. Время, в течение которого кьюбит находится в квантовом состоянии, называется временем когерентности [347]. В кьюбитах, основанных на переходах Джозефсона, для их ограждения от влияния окружающей среды их размещают в сверхпроводящие резонаторы [348]. Благодаря высокой добротности таких резонаторов электромагнитные колебания в них имеют большое время затухания, что позволяет значительно увеличить время когерентности кьюбитов [349] (рис. 19).

Пионерами и лидерами по разработкам элементов квантовой памяти является группа проф. Шоэлькопфа в Йельском Универнситете, США. В алюминимевых четверть-волновых резонаторах им удалось добиться добротности $Q \sim 10^{8}$ и времени когерентности несколько $\mathrm{ms}$ [350]. Особенностью работы резонаторов для квантовых компьютеров является крайне низкие температуры (около $10 \mathrm{mK}$ ), высокие частоты (несколько $\mathrm{GHz}$, для соблюдения условия $\hbar \omega>k T)$ и поля, соответствующие уровню одоного фотона $(\sim 1 \mathrm{mV} / \mathrm{m})$. В то же время, как было показано в предыдущем разделе, ниобиевые резонаторы для сверхпроводящих укорителей, работающих на более высоких температу$\operatorname{pax}(2 \mathrm{~K})$, более низких частотах $(<1.3 \mathrm{GHz})$ и гораздо более сильных полях ( $10 \mathrm{MV} / \mathrm{m})$, имеют добротность $Q \sim 10^{11}$. В связи с этим проводится ряд работ, направленных на адаптацию технологий обработки [351], формы [352] и методов изготовляения [353] ускоряющих резонаторов, включая перспективные технологии $3 D$-печати, для работы в режиме квантовых компьютеров. В настоящее время благодаря этому удалось достичь увеличения времени когерентности (до $2 \mathrm{~s}$ ) с помощью адаптированных резонаторов [354].

\section{Заключение}

Бурное развитие технологий в последнее десятилетие не обошло и ускорители заряженных частиц, интерес к которым растет с каждым годом. Разработки систем инспекции на основе новейших методов требуют источников излучения со сверхбыстрой перестройкой энергии, наличия непрерывных, монохроматических пучков или пучков тяжелых ионов. Ускорители, предназначенные для замены источников излучения на основе радиоактивных изотопов, должны иметь сравнимые размеры и стоимость. Современные медицинские системы требуют мобильности, достаточной для вращения вокруг пациента, быстрой доставки дозы в опухоль или скорости подстройки пучка более высокую, чем время нервных реакций пациента. Наконец, сверхпроводящие промышленные установки с высокой дозой облучения должны сравняться с нормальнопроводящими по сложности установки и обслуживания, чтобы их прикладное применение стало возможным. К сожалению, привычные технологии уже не способны решить вышеперечисленные проблемы.

Однако благодаря появлению более эффективных источников СВЧ-питания и их компонентов, работающих в сантиметровом и миллиметровом диапазонах, стала возможной миниатюризация ускорителей. Развитие лазерных технологий привело к значительному уменьшению габаритов систем питания для микроскопических плазменных ускорителей с ускоряющими градиентами, превосходящими самые высокие градиенты традиционных ускорителей на несколько порядков. Новые подходы к оптимизации формы ускоряющих структур, а также новых материалов (как на основе медных сплавов, так и сверхпроводящих) позволили повысить темпы ускорения, при этом увеличив энергетическую эффективность. Новые технологии изготовления ускоряющих структур, такие как, например, разделенные структуры, структуры с распределенным питанием и диэлектрические структуры, а в будущем и технология послойного синтеза $(3 D$-печати), позволили значительно упростить и удешевить процесс производства ускорителей. Кроме того, новые технологии неразъемных соединений, такие как электронно-лучевая сварка, диффузные и бесшовные методы, также внесли свой вклад в упрощение и повышение эффективности, а также показали пригодность для работы на высоких ускоряющих градиентах. Наконец, значительный прогресс в криогенных системах позволил достичь безжидкостного охлаждения как структур с небольшой (несколько W) тепловой нагрузкой до температур $4 \mathrm{~K}$, так и небольших структур до температур $10 \mathrm{mK}$, что открыло новые возможности для технологии квантовых компьютеров, чему также поспособствовали 
новейшие технологии, применяемые в сверхпроводящих ускорителях.

На примере конкретных разработок, охватывающих широкий спектр прикладных использований ускорителей, в работе были продемонстрированы основные тенденции в развитии прикладных ускорителей, а именно миниатюризация, вариативность параметров пучка и экономическая эффективность. Было показано как конкретные технологические новшества в области материаловедения, изготовления, измерений и теоретических исследований, позволяют реализовать эти тенденции в прототипах новейших ускорителей. Кроме того, был дан прогноз развития некоторых аспектов перспективных направлений, таких, как лазерное ускорение и квантовые компьютеры.

\section{Примечание}

Материал настоящей работы основан на информации, опубликованной в открытых источниках, и отображает персональный взгляд автора на современные тенденции развития ускорителей. В работе использованы некоторые заимствованные рисунки и фотографии, взятые из открытых источников. Рисунки, источники которых не приведены в тексте работы, принадлежат автору и RadiaBeam Technologies (https://radiabeam.com).

\section{Благодарности}

Автор выражает благодарность А. Муроху, А.Ю. Смирнову, М. Ruelas (RadiaBeam, США), проф. Н.П. Собенину (НИЯУ МИФИ, Москва), А. Пластуну (Мичиганский Государственный Университет MSU, США) и проф. В.И. Шведунову (НИИЯФ МГУ, Москва) за помощь в подготовке настоящей работы. Автором были использованы матералы совместной работы с А. Ародзеро, А.В. Смирновым, К. Талецким, S. Boucher, R. Agustsson, P. Frigola (RadiaBeam, CША), В. Долгашевым (Стэнфодская Ускорительная Лаборатория SLAC, CША), B. Mustapha, Z. Conway, M. Kelly, А. Жоленцом, J. Nolen, J. Power (Аргоннская Национальная Лаборатория ANL, CША), проф. A. Cleland (Университет Чикаго, США), проф. П.Н. Остроумовым (Мичиганский Государственный Университет MSU, CША), C. Johnsotne, А. Романенко, В.П. Яковлевым (Национальная Ускорительная Лаборатория Ферми, США), проф. R. Lanza, проф. R. Temkin (Массачусетский Технологический Университет, США), проф. М.А. Гусаровой (НИЯУ МИФИ, Москва) и др.

\section{Конфликт интересов}

Автор заявляет, что у него нет конфликта интересов.

\section{Список литературы}

[1] R.W. Garnett. Phys. Procedia, 66, 196 (2015). DOI: $10.1016 /$ j.phpro.2015.05.026

[2] V.N. Starovoitova, L. Tchelidze, D.P. Wells. Appl. Radiat. Isot., 85, 39 (2014). DOI: 10.1016/j.apradiso.2013.11.122

[3] W.P. Levin, H. Kooy, J.S. Loeffler, T.F. DeLaney. Br. J. Cancer., 93 (8), 849 (2005). DOI: $10.1038 /$ sj.bjc. 6602754

[4] T.D. Malouff, A. Mahajan, S. Krishnan, C. Beltran, D.S. Seneviratne, D.M. Trifiletti. Front. Oncol., 10, 82 (2020). DOI: $10.3389 /$ fonc. 2020.00082

[5] J.F. Diehl. Radiat. Phys. Chem., 62 (3-6), 211 (2002). DOI: $10.1016 / \mathrm{S} 0969-806 \mathrm{X}(01) 00622-3$

[6] K. Hossain, Y. Avasn Maruthi, N. Lakshmana Das, K.P. Rawat, K.S.S. Sarma. Appl. Water Sci., 8 (6), 1 (2018). DOI: $10.1007 / \mathrm{s} 13201-018-0645-6$

[7] P.A. Bystrov, Y.S. Pavlov, O.V. Souvorova, I.Yu. Yakupov. Radiat. Phys. Chem., 161, 83 (2019). DOI: 10.1016/j.radphyschem.2019.03.053

[8] P.A. Bystrov, A.V. Gordeev, A.Yu. Kolokolova, M.A. Zavyalov, N.V. Ilyukhina, A.A. Molin, Y.S. Pavlov, S.P. Polyakova, A.V. Prokopenko, V.P. Filippovich. Phys. Atom. Nuclei, 81 (10), 1526 (2018). DOI: $10.1134 / \mathrm{S} 1063778818110054$

[9] Ю.С. Павлов. В сб.: Труды XXIV Межжуународной конференции „Радиащионная физика твердого тела“ под ред. Г.Г. Бондаренко (ФГБНУ „НИИ ПМТ“, М., 2014) c. 22.

[10] S. Boucher, S. Kutsaev, A. Murokh. In: APS Division of Physics of Beams, Annual Newsletter Ed. by M. Minty (APS Physics Publishing, USA, 2019) p. 24.

[11] Б.Ю. Богданович, А.В. Нестерович, А.Е. Шиканов, М.Ф. Ворогушин, Ю.А. Свистунов. Дистанционный радиационный контроль с линейными ускорителями, в 2 т. (Энергоатомиздат, М, 2009) ISBN: 978-5-283-03290-0

[12] V.Y. Yu, A. Landers, K. Woods, D. Nguyen, M. Cao, D. Du, R.K Chin, K. Sheng, T.B. Kaprealian. Int. J. Radiat. Oncol. Biol. Phys., 101 (1), 144 (2018). DOI: 10.1016/j.ijrobp.2018.01.048

[13] P. Symonds, G.D.D. Jones. Clin. Oncol. 31 (7), 405 (2019). DOI: 10.1016/j.clon.2019.05.011

[14] G. Moroff, N.L. Luban. Transfus. Med. Rev., 11 (1), 15 (1997). DOI: 10.1016/S0887-7963(97)80006-5

[15] S.M. Telesz, J.M. Gomez. In: Proc. 18th World Conference on Nondestructive Testing (Durban, South Africa, 2012), p. 611.

[16] J.R. Fanchi. Integrated Reservoir Asset Management: Principles and Best Practices (Gulf Professional Publishing, Houston, 2010) 376 p. ISBN: 978-0-12-382088-4

[17] J.F. Cameron, C.G. Clayton. Radioisotope Instruments. A volume in International Series of Monographs in Nuclear Energy (Pergamon, Oxford, 1971) 320 p. ISBN: 978-1483126913

[18] G.M. Moore, M.A. Pomper. Permanent Risk Reduction: A Roadmap for Replacing High-Risk Radioactive Sources and Materials (The President and Trustees of Middlebury College, Monterey, 2015). ISBN: 978-0-9892361-9-5

[19] N.P. Sobenin, B.V. Zverev. Electrodynamical Characteristics of Accelerating Cavities, first ed. (CRC Press, London, 1999). ISBN: $978-9069940038$ 
[20] T. Wangler. Principles of RF Linear Accelerators (Wiley, New York, 1998). ISBN: 978-3527618408

[21] L.W. Brady, T.E. Yaeger. Encyclopedia of Radiation Oncology (Springer, Berlin, Heidelberg, 2013). ISBN: 9783-540-85513-2

[22] S. Hanna. $R F$ Linear Accelerators for Medical and Industrial Applications, $1^{\text {st }}$ ed. (Artech House, 2012) p. 216. ISBN: 978-1608070909

[23] V.I. Shvedunov, A.S. Alimova, A.N. Ermakova, A.N. Kamanina, V.V. Khankina, A.S. Kurilika, L.Yu. Ovchinnikova, N.I. Pakhomov, N.V. Shvedunov, D.S. Yurov, I.V. Shvedunov, A.S. Simonov. Radiat. Phys. Chem., 159, 95 (2019). DOI: 10.1016/j.radphyschem.2019.02.044

[24] S.V. Kutsaev, N.P. Sobenin, A.A. Anisimov, M. Ferderer, A.A. Zavadtsev, A.A. Krasnov. In Proc. of LINAC08 (Victoria, BC, Canada, 2018), p. THP058.

[25] L. Ovchinnikova, V. Shvedunov. In Proc. of 29th Linear Accelerator Conference, LINAC2018 (Beijin, China, 2018), p. TUPO097. DOI: 10.18429/JACoW-LINAC2018TUPO097

[26] S.V. Kutsaev, R. Agustsson, A. Arodzero, S. Boucher, P. Burstein, A.Yu. Smirnov. AIP Conf. Proc., 2160, 050014 (2019). DOI: $10.1063 / 1.5127706$

[27] VMA 1647G Specifications in: https://www.cpii.com/docs/ datasheets/16/VMA1647G\%20ATC\%20MAGNETRON $\% 20 \mathrm{NF} \% 205-5-20 . p d f$.

[28] M. Dal Forno, V. Dolgashev, G. Bowden, C. Clarke, M. Hogan, D. McCormick, A. Novokhatski, B. Spataro, S. Weathersby, S.G. Tantawi. Phys. Rev. Accel. Beams, 19, 01130 (2016). DOI: 10.1103/PhysRevAccelBeams.19.011301

[29] E.M. Choi, C. Marchewka, I. Mastovsky, M.A. Shapiro, J.R. Sirigiri. J. Phys.: Conf. Ser., 25 (1), 1 (2005). DOI: $10.1088 / 1742-6596 / 25 / 1 / 001$

[30] M.A.K. Othman, J. Picard, S. Schaub, V.A. Dolgashev, S.M. Lewis, J. Neilson, A. Haase, S. Jawla, B. Spataro, R.J. Temkin, S. Tantawi, E.A. Nanni. Appl. Phys. Lett., 117 (7), 073502 (2020). DOI: 10.1063/5.0011397

[31] S.V. Kutsaev, B. Jacobson, A.Yu. Smirnov, T. Campese, V.A. Dolgashev, V. Goncharik, M. Harrison, A. Murokh, E. Nanni, J. Picard, M. Ruelas, S.C. Schaub. Phys. Rev. Appl., 11 (3), 034052 (2019). DOI: 10.1103/PhysRevApplied.11.034052

[32] A.V. Smirnov, R. Agustsson, W.J. Berg, J. Dooling, T. Campese, Y. Chen, D. Gavryushkin, L. Erwin, J. Hartzell, R. Keane, F.H. O’Shea, E. Spranza, S. Pasky, M. Ruelas, Y. Sun, A.A. Zholents. Nucl. Instrum. Methods Phys. Res. Sect. A, 960, 163655 (2020). DOI: $10.1016 /$ j.nima.2020.163655

[33] S. Antipov, C. Jing, P. Schoessow, A. Kanareykin, V. Yakimenko, A. Zholents, W. Gai. Rev. Sci. Instrum., 84, 022706 (2013). DOI: $10.1063 / 1.4790432$

[34] W. Wuensch. CERN Courier, 23 (2018). Available at: https://cerncourier.com/a/high-gradient-x-band-technologyfrom-tev-colliders-to-light-sources-and-more/

[35] A. Zholents, W. Gai, S. Doran, R. Lindberg, J.G. Power, N. Strelnikov, Y. Sun, E. Trakhtenberg, I. Vasserman, C. Jing, A. Kanareykin, Y. Li, Q. Gao, D.Y. Shchegolkov, E.I. Simakov. Nucl. Instrum. Methods Phys. Res. A, 829, 190 (2016). DOI: 10.1016/j.nima.2016.02.003
[36] S. Antipov, C. Jing, M. Fedurin, W. Gai, A. Kanareykin, K. Kusche, P. Schoessow, V. Yakimenko, A. Zholents. Phys. Rev. Lett., 108, 144801 (2012), DOI: 10.1103/PhysRevLett.108.144801

[37] A. Grudiev, S. Calatroni, W. Wuensch. Phys. Rev. Accel. Beams, 12, 102001 (2009). DOI: 10.1103/PhysRevSTAB.12.102001

[38] D.P. Pritzkau, R.H. Siemann. Phys. Rev. Accel. Beams, 5, 112002 (2002). DOI: 10.1103/PhysRevSTAB.5.112002

[39] V.A. Dolgashev, L. Faillace, Y. Higashi, A. Marcelli, B. Spataro R. Bonifazi. J. Instrum., 15, P01029 (2020). DOI: $10.1088 / 1748-0221 / 15 / 01 / \mathrm{P} 01029$

[40] J.B. Rosenzweig, A. Cahill, V. Dolgashev, C. Emma, A. Fukasawa, R. Li, C. Limborg, J. Maxson, P. Musumeci, A. Nause, R. Pakter, R. Pompili, R. Roussel, B. Spataro, S. Tantawi. Phys. Rev. Accel. Beams, 22, 023403 (2019). DOI: 10.1103/PhysRevAccelBeams.22.023403

[41] A.D. Cahill, J.B. Rosenzweig, V.A. Dolgashev, S.G. Tantawi, S. Weathersby. Phys. Rev. Accel. Beams, 21, 102002 (2018). DOI: doi.org/10.1103/PhysRevAccelBeams.21.102002

[42] D. Satoh, T. Shibuya, H. Ogawa, M. Tanaka, R. Kuroda, S. Mori, M. Yoshida, H. Toyokawa. Nucl. Instrum. Methods Phys. Res. Sect. B, 459, 148 (2019). DOI: 10.1016/j.nimb.2019.09.006

[43] Advanced Accelerator Development Strategy Report: DOE Advanced Accelerator Concepts Research Roadmap Workshop, ed. by T. Antonsen (Washington D.C., 2016).

[44] A.E. Hussein, N. Senabulya, Y. Ma, M.J.V. Streeter, B. Kettle, S.J.D. Dann, F. Albert, N. Bourgeois, S. Cipiccia, J.M. Cole, O. Finlay, E. Gerstmayr, I. Gallardo González, A. Higginbotham, D.A. Jaroszynski, K. Falk, K. Krushelnick, N. Lemos, N.C. Lopes, C. Lumsdon, O. Lundh, S.P.D. Mangles, Z. Najmudin, P.P. Rajeev, C.M. Schlepütz, M. Shahzad, M. Smid, R. Spesyvtsev, D.R. Symes, G. Vieux, L. Willingale, J.C. Wood, A.J. Shahani, A.G.R. Thomas. Sci. Rep., 9, 3249 (2019). DOI: 10.1038/s41598-019-39845-4

[45] E. Gschwendtner, P. Muggli. Nat. Rev. Phys., 1, 246 (2019). DOI: $10.1038 / \mathrm{s} 42254-019-0049-\mathrm{Z}$

[46] M.C. Thompson, H. Badakov, A.M. Cook, J.B. Rosenzweig, R. Tikhoplav, G. Travish, I. Blumenfeld, M.J. Hogan, R. Ischebeck, N. Kirby, R. Siemann, D. Walz, P. Muggli, A. Scott, R.B. Yoder. Phys. Rev. Lett., 100, 214801 (2008). DOI: 10.1103/PhysRevLett.100.214801

[47] F. Grüner. Physics, 12, 1 (2019). DOI: 0.1103/Physics.12.19

[48] M. Nishiuchi, H. Sakaki, T. Hori, P.R. Bolton, K. Ogura, A. Sagisaka, A. Yogo, M. Mori, S. Orimo, A.S. Pirozhkov, I. Daito, H. Kiriyama, H. Okada, S. Kanazawa, S. Kondo, T. Shimomura, M. Tanoue, Y. Nakai, H. Sasao, D. Wakai, H. Daido, K. Kondo, H. Souda, H. Tongu, A. Noda, Y. Iseki, T. Nagafuchi, K. Maeda, K. Hanawa, T. Yoshiyuki, T. Shirai. In: Proc. of IPAC'10 Conf. (Kyoto, Japan, 2010) p. 88.

[49] B.M. Hegelich, D. Jung, B. J. Albright, M. Cheung, B. Dromey, D.C. Gautier, C. Hamilton, S. Letzring, R. Munchhausen, S. Palaniyappan, R. Shah, H.-C. Wu, L. Yin, J.C. Fernández. arXiv:1310.8650 [physics.plasm-ph] (2013).

[50] N.V. Sapra, K.Y. Yang, D. Vercruysse, K.J. Leedle, D.S. Black, R.J. England, L. S1, R. Trivedi, Y. Miao, O. Solgaard, R.L. Byer, J. Vučković. Science, 367 (6473), 79 (2020). DOI: 10.1126/science.aay5734

[51] J. Levesque, P.B. Corkum. Can. J. Phys., 84 (1), 1 (2006). DOI: 10.1139/P05-068 
[52] S. Machida, R. Barlow, J.S. Berg, N. Bliss, R.K. Buckley, J.A. Clarke, M.K. Craddock, R. D’Arcy, R. Edgecock, J.M. Garland, Y. Giboudot, P. Goudket, S. Griffiths, C. Hill, S.F. Hill, K.M. Hock, D.J. Holder, M.G. Ibison, F. Jackson, S.P. Jamison, C. Johnstone, J.K. Jones, L.B. Jones, A. Kalinin, E. Keil, D.J. Kelliher, I.W. Kirkman, S. Koscielniak, K. Marinov, N. Marks, B. Martlew, P.A. McIntosh, J.W. McKenzie, F. Méot, K.J. Middleman, A. Moss, B.D. Muratori, J. Orrett, H.L. Owen, J. Pasternak, K. J. Peach, M.W. Poole, Y-N. Rao, Y. Saveliev, D.J. Scott, S.L. Sheehy, B.J.A. Shepherd, R. Smith, S.L. Smith, D. Trbojevic, S. Tzenov, T. Weston, A. Wheelhouse, P.H. Williams, A. Wolski, T. Yokoi. Nat. Phys., 8 (3), 243 (2012). DOI: doi.org/10.1038/nphys 2179

[53] Beam Dynamics Newsletter 43, ed. by C.R. Prior, (International Committee for Future Accelerators, USA, 2007).

[54] H. Aït Abderrahim, J. Galambos, Y. Gohar, S. Henderson, G. Lawrence, T. McManamy, A.C. Mueller, S. Nagaitsev, J. Nolen, E. Pitcher, R. Rimmer, R. Sheffield, M. Todosow. Accelerator and Target Technology for Accelerator Driven Transmutation and Energy Production, Report number: FERMILAB-FN-0907-DI, LA-UR-10-06754, (Fermilab, USA, 2010)

[55] S.V. Kutsaev, Z.A. Conway, P.N. Ostroumov, C.J. Johnstone, R.D. Ford. In: Proc. of Cyclotrons 2013 Conf. (Vancouver, BC, Canada, 2013) p. MO3PB03.

[56] Б.А. Шиляев, Л.С. Ожигов, А.А. Пархоменко. Препр. Нац. науч. центр „Харьк. физ.-техн. ин-т“ НАН Украины, 23 c. (X., 2006).

[57] Л.Г. Дубас. МЖПФИ, 3, 7 (2019). DOI: $10.17513 / \mathrm{mjpfi} .12675$

[58] S. Kutsaev, R. Agustsson, A. Arodzero, S. Boucher, J. Hartzell, A. Murokh, F. O’Shea, A.Yu. Smirnov. Phys. Procedia, 90, 115 (2017). DOI: doi.org/10.1016/j.phpro.2017.09.036

[59] A. Arodzero, S. Boucher, J. Hartzell, S.V. Kutsaev, R.C. Lanza, V. Palermo, S. Vinogradov, V. Ziskin. In Proc. of 2015 IEEE Nuclear Science Symposium and Medical Imaging Conference (NSS/MIC) (San Jose, CA, USA, 2015), p. 16356934. DOI: 10.1109/NSSMIC.2015.7581836

[60] S. Ogorodnikov, V. Petrunin, Phys. Rev. Accel. Beams 5, 104701 (2002). DOI: 10.1103/PhysRevSTAB.5.104701

[61] A.A. Zavadtsev, D.A. Zavadtsev, A.A. Krasnov, N.P. Sobenin, S.V. Kutsaev, D.V. Churanov, M.O. Urbant. Instrum. Exp. Tech., 54 (2), 241 (2011). DOI: $10.1134 / \mathrm{S} 0020441211020114$

[62] S. Benedetti. In Proc. of 29th International Linear Accelerator Conference, (Beijing, China, 2018) p. TH1P03.

[63] T. Kubiak. Brit. J. Radiol., 89, 1066 (2016). DOI: $10.1259 /$ bjr.20150275

[64] B.M. Oborn, S. Dowdell, P.E. Metcalf, S. Crozier, R. Mohan, P.J. Keall. Med. Phys., 44 (8), 77 (2017). DOI: $10.1002 / \mathrm{mp} .12371$

[65] B. Mustapha, B. Aydogan, J. Nolen, A. Nassiri, J. Noonan, M. Pankuch, J. Welsh, R. Schulte, J. Robb. AIP Conf. Proc., 2160, 050009 (2019). DOI: 10.1063/1.5127701

[66] V. Khankin, A. Alimov, A. Ermakov, A. Kamanin, A. Kurilik, N. Pakhomov, I. Shvedunov, N. Shvedunov, V. Shvedunov, A. Simonov, D. Yurov. In Proc. of 29th Linear Accelerator Conference, LINAC2018 (Beijing, China, 2018) p. MOPO062. DOI: 10.18429/JACoW-LINAC2018MOPO062
[67] P. Apiwattanakul, S. Rimjaem. Nucl. Instrum. Methods Phys. Res. B, 466, 69 (2020). DOI: 10.1016/j.nimb.2020.01.012

[68] B. Hidding, O. Karger, T. Königstein, G. Pretzler, G.G. Manahan, P. McKenna, R. Gray, R. Wilson, S.M. Wiggins, G.H. Welsh, A. Beaton, P. Delinikolas, D.A. Jaroszynski, J.B. Rosenzweig, A. Karmakar, V. FerletCavrois, A. Costantino, M. Muschitiello, E. Daly. Sci. Rep., 7, 42354 (2017). DOI: 10.1038/srep42354

[69] J.H.E. Yong, T. McGowan, R. Redmond-Misner, J. Beca, P. Warde, E. Gutierrez, J.S. Hoch. Curr. Oncol. 23 (3), 228 (2016). DOI: $10.3747 /$ co.23.2998

[70] Ю.С. Павлов. Тез. докл. Научной сессии НИЯУ МИФИ. Т 2. (МИФИ, М., 2013), с. 126.

[71] M.R. Cleland. In CAS-CERN Accelerator School: small accelerators, pp. 383-416 (2006). DOI: 10.5170/CERN2006-012.383

[72] C. Körner. Int. Mater. Rev., 61 (5), 361 (2014). DOI: 10.1080/09506608.2016.1176289.

[73] Y. Li, B. Pan, M M. Tentzeris, J. Papapolymerou. In Proc. 2007 IEEE/MTT-S International Microwave Symposium (Honolulu, HI, USA, 2007) p. 1031-1034. DOI: 10.1109/MWSYM.2007.380233

[74] R. Agustsson, S. Boucher, S. Kutsaev. World Patent Application WO2018222839A1, 2018.

[75] R.M. Sundelin, R.M. Sundelin, J.L. Kirchgessner, M. Tigner. IEEE Trans. Nucl. Sci., 24 (3), 1686 (1977). DOI: $10.1109 /$ TNS.1977.4329052

[76] O.N. Brezhnev, V.M. Pavlov, O.V. Pirogov, Ju.D. Chernousov. Probl. Atom. Sci. Tech., 3, 65 (2001). URI: http://dspace.nbuv.gov.ua/handle/123456789/79238

[77] S. Tantawi, M. Nasr, Z. Li, C. Limborg, P. Borchard. arXiv:1811.09925 [physics.acc-ph] (2018).

[78] M. Weglowski, S. Błacha, A. Phillips. Vacuum, 130, 72 (2016). DOI: 10.1016/j.vacuum.2016.05.004

[79] J.W. Elmer, J. Klingmann, K. Van Bibber. Phys. Rev. Accel. Beams, 4, 053502 (2001). DOI: $10.1103 /$ PhysRevSTAB.4.053502

[80] S. Antipov, R. Kostin, S. Kuzikov, A. Vikharev. In Proc. of 9th International Particle Accelerator Conf., (Vancouver, BC, Canada, 2018), pp. WEPMF068. DOI: 10.18429/JACoW-IPAC2018-WEPMF068

[81] J. Scifo, A. Marcelli, B. Spataro, D. Hampai, S. Dabagov, S. Sarti, A. Di Trolio, R. Moscatelli, S. Macis, L. Faillace. Instruments, 3 (4), 61 (2019). DOI: 10.3390/instruments3040061

[82] S.V. Kutsaev, R. Agustsson, A. Arodzero, S. Boucher, A. Murokh, A.Yu. Smirnov. Nucl. Instrum. Methods Phys. Res. B, 459, 179 (2019). DOI: 10.1016/j.nimb.2019.08.029

[83] G. Dome. In Linear Accelerators, ed. P. Lapostolle (Ams. North-Holland Publ. Co., 1970), ASIN: B0006C071O

[84] M. Nasr, S. Tantawi. In Proc. of 9th International Particle Accelerator Conf. IPAC2018 (Vancouver, BC, Canada, 2018), pp. THPMK049. DOI: 10.18429/JACoW-IPAC2018-THPMK049

[85] S.P. Antipov, V. Dolgashev, R. Kostin. In Proc. of 9th International Particle Accelerator Conf. IPAC2018 (Vancouver, BC, Canada, 2018), pp. WEPMF06907. DOI: 10.18429/JACoW-IPAC2018-WEPMF069

[86] V.A. Dolgashev. In Proc. of IEEE PAC 2003 Conf. (Portland, Oregon, USA, 2003) p. 12671269. DOI: 10.1109/PAC.2003.1289674

[87] Copper Wire Tables, (Technical report), Circular of the Bureau of Standards No.31 (3d ed.). (National Bureau of Standards, Washington, D. C. Inst. of Applied Technology, 1914). NTIS Issue Number: 196610. 
[88] Radiofrequency Accelerator R\& D Strategy Report (U.S. Department of Energy, Washington D.C., USA, 2017). Available at: https://www.osti.gov/servlets/purl/1631119.

[89] A. Danagoulian, W. Bertozzi, C.L. Hicks, A.V. Klimenko, S.E. Korbly, R.J. Ledoux, C.M. Wilson. In Proc. 2010 IEEE International Conference on Technologies for Homeland Security (HST) (Waltham, MA, USA, 2010) p. 379. DOI: $10.1109 /$ THS.2010.5654938

[90] A.J. Berejka. Radiat. Phys. Chem., 46 (4-6), 429 (1995). DOI: 10.1016/0969-806X(95)00188-4

[91] S.M. Seltzer, J.P. Farrell, J. Silverman. IEEE Trans. Nucl. Sci., 30 (2), 1629 (1983). DOI: 10.1109/TNS.1983.4332602

[92] J.D. Hunt, G. Alliger. Radiat. Phys. Chem., 14(1-2), 39 (1979). DOI: 10.1016/0146-5724(79)90010-4

[93] E.W. Bennett. Radiat. Phys. Chem., 14 (3-6), 947 (1979). DOI: $10.1016 / 0146-5724(79) 90132-8$

[94] B.P. Fairand. Radiation Sterilization for Health Care Products, X-Ray, Gamma, and Electron Beam (CRC Press, Boca Raton, Florida, 2002), ISBN: 978-1587160745.

[95] A.Yu. Gracheova, M.A. Zav'yalov, N.V. Ilyukhina, V.A. Kukhto, V.T. Tarasyuk, V.P. Filippovich, A.V. Egorkin, A.V. Chasovskikh, Yu.S. Pavlov, A.V. Prokopenko, N.E. Strokova, S.A. Artem'ev, S.P. Polyakova. Phys. Atom. Nuclei, 79 (14), 1682 (2016). DOI: $10.1134 / \mathrm{S} 1063778816140118$

[96] Y.S. Pavlov, A.A Revina, O.V. Souvorova, N.L. Voropaeva, D.V. Chekmar, E.V. Abkhalimov, M.A. Zavyalov, V.P. Filippovich. J. Phys. Conf. Ser., 941, 012098 (2017). DOI: 10.1088/1742-6596/941/1/012098

[97] A.G. Chmielewsk, E. Iller, B. Tyminski, Z. Zimek, J. Licki. Mod. Power Syst., 53 (2002). Available at: https:/www.modernpowersystems.com/features/featurefluegas-treatment-by-electron-beam-technology

[98] H. Podlech. CERN Yellow Report CERN-2013-001, pp. 151-170 (2013). DOI: 10.5170/CERN-2013-001.151

[99] H. Padamsee. RF Superconductivity: Science, Technology, and Applications (Wiley-VCH Verlag GmbH \& Co. KGaA, Weinheim, 2009). ISBN: 978-3-527-40572-5.

[100] R. Nagimov, I. Bylinsky, D. Kishi, S. Koscielniak, A. Koveshnikov, R. Laxdal, D. Yosifov. In Proc. 9th International Particle Accelerator Conf. IPAC2018 (Vancouver, BC, Canada, 2018), pp. THPAL121. DOI: 10.18429/JACoW-IPAC2018-THPAL121

[101] R. Radebaugh. J. Phys.: Condens. Matter, 21, 164219 (2009). DOI: $10.1088 / 0953-8984 / 21 / 16 / 164219$

[102] W.E. Gifford. In Advances in Cryogenic Engineering, ed. by K.D. Timmerhaus vol. 11 (Springer, Boston 1966), p. 152159. DOI: $10.1007 / 978-1-4757-0522-5 \_16$

[103] A. de Waele. J. Low Temp. Phys., 164 (5-6), 179 (2011). DOI: doi.org/10.1007/s10909-011-0373-X

[104] S. Posen, D.L. Hall. Supercond. Sci. Technol., 30 (3), 033004 (2017). DOI: 10.1088/1361-6668/30/3/033004

[105] T. Tan, M. A. Wolak, X.X. Xi, T. Tajima, L. Civale. Sci. Rep. 6, 35879 (2016). DOI: 10.1038/srep35879

[106] R.C. Dhuley, R. Kostin, O. Prokofiev, M.I. Geelhoed, T.H. Nicol, S. Posen, J.C.T. Thangaraj, T.K. Kroc, R.D. Kephart. IEEE Trans. Appl. Supercond., 29(5), 0500205 (2019). DOI: 10.1109/TASC.2019.2901252

[107] J.Y. Benitez, A. Donoghue, M.B. Johnson, W. Lu, B. Ninemire, L. Phair, D.S. Todd, D. Xie. In Proc. of 2017 IEEE Radiation Effects Data Workshop (REDW) (New Orleans, LA, USA, 2017) pp. 1-5.

DOI: $10.1109 /$ NSREC.2017.8115438
[108] T.C. Kaspar, C.A. Lavender, M.W. Dibert. Report \#PNNL26490 (Pacific Northwest National Laboratory Richland, Washington, USA, 2017).

[109] J. Kang, F.N. von Hippel. Sci. Global Secur., 9 (1), 1 (2001).

[110] A.S. Erickson. Thesis (Ph. D.), Massachusetts Institute of Technology, Boston, MA, USA, 2011.

[111] J. Bendahan. Nucl. Instrum. Methods Phys. Res. A, 954, 161120 (2020). DOI: 10.1016/j.nima.2018.08.079

[112] A.M.I. Alsayyed, K.J. Dale, J.F. Diehl, J. Farkas, M. Frissel, H. Fröhlich, J.H. Hubbel, J.R. Lujan, G. Pauli, K.W. Bögl, A. Brynjolfsson, F.K. Käferstein, A.-M. Schmitt-Hannig, R.B. Singh, H. Stiff, E. Casadei, G. Geus, C. Koch, C.T. Blunden, G. Bennet, C.S. Nunan. Bull. World Health Org. 68 (3), 297 (1990).

[113] Д.О. Спирин, Я.А. Бердников, Ю.Н. Гавриш. Научнотехнические ведомости СПбГПУ, 2 (98), 120 (2010).

[114] W.G.J. Langeveld. AIP Conf. Proc., 2160, 050018 (2019). DOI: $10.1063 / 1.5127710$

[115] W.G.J. Langeveld. Phys. Procedia, 90, 291 (2017). DOI: 10.1016/j.phpro.2017.09.014

[116] J. Callerame. Powder Diffr., 21 (2), 132 (2006). DOI: https://doi.org/10.1154/1.2204054

[117] A. Arodzero, S. Boucher, P. Burstein, S.V. Kutsaev, R.C. Lanza, V. Palermo. In Proc. of 2019 IEEE International Symposium on Technologies for Homeland Security (HST) (Woburn, MA, USA, 2019) p. 1. DOI: 10.1109/HST47167.2019.9032928

[118] Z. An, L. Tian, J. Zhu, M. Liua. Data Brief., 17, 744 (2018). DOI: $10.1016 /$ j.dib.2018.01.111

[119] A. Arodzero. US Patent Application US20110163236A1 (2011).

[120] A. Arodzero, S. Boucher, J. Hartzell, S.V. Kutsaev, R.C. Lanza, V. Palermo, S. Vinogradov, V. Ziskin. In Proc. of IEEE-2015 Nuclear Science Symposium and Medical Imaging Conf. (San Diego, CA, USA. 2015) p. 1. DOI: $10.1109 /$ NSSMIC.2015.7581836

[121] A. Arodzero, S.V. Kutsaev, V. Ziskin. US Patent 10481113, 20170336526 (22017).

[122] I. Gadjev, N. Sudar, M. Babzien, J. Duris, P. Hoang, M. Fedurin, K. Kusche, R. Malone, P. Musumeci, M. Palmer, I. Pogorelsky, M. Polyanskiy, Y. Sakai, C. Swinson, O. Williams, J.B. Rosenzweig. Sci. Rep., 9, 532 (2019). DOI: 10.1038/s41598-018-36423-y

[123] S.V. Kutsaev, R. Agustsson, A. Arodzero, S. Boucher, L. Faillace, J. Hartzell, V. Ziskin. In Proc. of IEEE-2015 Nuclear Science Symposium and Medical Imaging Conf. (San Diego, CA, USA, 2015), pp. 1-7. DOI: 10.1109/NSSMIC.2015.7581765

[124] Vehicle Weight Classes \& Categories, United States Department of Energy document, available at: https://afdc.energy.gov/data/10380

[125] J. Hašek. X-Ray and Neutron Structure Analysis in Materials Science (Springer, Boston, MA, 1989) ISBN: 9781-4613-0767-9

[126] S.V. Kutsaev, R. Agustsson, A. Arodzero, R. Berry, S. Boucher, Y. Chen, J. Hartzell, B. Jacobson, A. Laurich, A. Murokh, E. Savin, A.Yu. Smirnov, A. Verma. In Proc. of NAPAC'16 Conf., (Chicago, IL, USA, 2016), p. TUPOA68. DOI: 10.18429/JACoW-NAPAC2016TUPOA68

[127] О.А. Вальднер, А.Д. Власов, А.В. Шальнов. Линейные ускорители (Атомиздат, М, 1969).

[128] В. Москалев, Г. Сергеев. Индукционный ускоритель электронов - бетатрон (Изд-во ТПУ, Томск, 2014) 
[129] S. Chakhov, S.V. Kasyanov, V.A. Kasyanov, S.P. Osipov, M.M. Stein, A.M. Stein, S. Xiaoming. J. Phys.: Conf. Ser., 671, 012024 (2016). DOI: 10.1088/1742-6596/671/1/012024

[130] B.V. Solomatin. Sov. Phys. J., 13, 1046 (1970). DOI: $10.1007 / \mathrm{BF} 00818565$

[131] A. Arodzero, S. Boucher, S.V. Kutsaev, R.C. Lanza, V. Palermo, F. O’Shea, V. Ziskin. In Proc. of 2016 IEEE Nuclear Science Symposium, Medical Imaging Conference and Room-Temperature Semiconductor Detector Workshop (NSS/MIC/RTSD) (Strasbourg, France, 2016) p. 1. DOI: 10.1109/NSSMIC.2016.8069705

[132] W. Bertozzi, R.J. Ledoux. US Patent US8718219B2 (2014).

[133] J.M. Bassaler, J.M. Capdevila, O. Gal, F. Lainé, A. Nguyen, J.P. Nicolaï, K. Umiastowski. Nucl. Instrum. Methods Phys. Res. B, 68 (1-4), 92 (1992). DOI: $10.1016 / 0168-583 X(92) 96056-5$

[134] V.I. Shvedunov, A.S. Alimov, A.S. Chepurnov, O.V. Chubarov, I.V. Gribov, B.S. Ishkhanov, I.V. Surma, A.V. Tiunov. In Proc. of 1993 Particle Accelerator Conf. (Washington, DC, USA, USA, 1993) p. 2059 (1993).

DOI: 10.1109/PAC.1993.309222

[135] P.H. Debenham, S. Penner, R.L. Ayres, R.I. Cutler, E.R. Lindstrom, D.L. Mohr, J.E. Rose, M.A.D. Wilson, N.R. Yoder, L.M. Young, R.E. Martin, A. Mitra, J.M. Potter, R.H. Stokes, P.J. Tallerico, L. Wilkerson. IEEE Trans. Nucl. Sci., 30 (2), 1391 (1983). DOI: 10.1109/TNS.1983.4332540

[136] A.N. Ermakov, B.S. Ishkhanov, A.N. Kamanin, N.I. Pakhomov, V.V. Khankin, V.I. Shvedunov, N.V. Shvedunov, E.E. Zhuravlev, A.I. Karev, N.P. Sobenin. Instrum. Exp. Tech., 61, 173 (2018). DOI: https://doi.org/10.1134/S0020441218020136

[137] В.И. Векслер. УФН, 93, 521 (1967). 10.3367/UFNr.0093.196711k.0521

[138] A.V. Smirnov, R. Agustsson, R. Berry, S. Boucher, Y. Chen, S. Kutsaev, F. O'Shea. Nucl. Instrum. Methods Phys. Res. A, 953, 163160 (2020). DOI: 10.1016/j.nima.2019.163160

[139] A. Jankowiak. Eur. Phys. J. A, 28, 149 (2006). DOI: $10.1140 /$ epja/i2006-09-016-3

[140] E.S. Marshall. Thesis (Master) (Massachusetts Institute of Technology, Boston, 2012)

[141] J. Medalia. Detection of Nuclear Weapons and Materials: Science, Technologies, Observations (BiblioGov, USA, 2013). ISBN: 978-1295023080

[142] P.B. Rose, A.S. Erickson. Phys. Rev. C, 97, 064305 (2018). DOI: 10.1103/PhysRevC.97.064305

[143] J. Nattress, F. Sutanto, P.-W. Fang, Y.-Z. Chen, A. Cheng, K.-Y. Chu, T.-S. Duh, H.-Y. Tsai, M.-W. Lin, I. Jovanovic. arXiv:1911.05458v1 [nucl-ex] (2019).

[144] L. Smith. Nucl. Instrum. Meth., 18-19, 397 (1962). DOI: $10.1016 / \mathrm{S} 0029-554 \mathrm{X}(62) 80050-0$

[145] P.W. Schmor. Rev. Accel. Sci. Technol., 4, 103 (2011). DOI: https://doi.org/10.1142/S1793626811000574

[146] А.Н. Лебедев, А.В. Шальнов. Основы бизики и техники ускорителей, 2-е изд., перераб. и доп. (Энергоатомиздат, M, 1991), $528 \mathrm{c}$.

[147] J. Vincent, G. Blosser, G. Horner, K. Stevens, N. Usher, X. Wu, S. Vorozhtsov, V. Smirnov. In Proc. of the 21st Int. Conf. on Cyclotrons and Their Applications, Cyclotrons2016, (Switzerland, 2016), p. THB02.

[148] H.H. Tsai, H.C. Li, F.Z. Hsiao, S.H. Chang, W.S. Chiou. In. Proc. of EPACO8 (Genoa, Italy, 2008), p. WEPD044.

[149] S. Zaremba, W. Kleeven. CERN Yellow Report CERN-2017004-SP p. 177 (2017). DOI: 10.23730/CYRSP-2017-001.177
[150] J. Schippers. CERN Yellow Report CERN-2017-004-SP pp. 165-175 (2017). DOI: 10.23730/CYRSP-2017-001.165

[151] A.L Radovinsky. US Patent US10028369B2 (2018).

[152] V. Smirnov, S. Vorozhtsov. Phys. Part. Nuclei 47 (5), p. 863 883 (2016). DOI: 10.1134/S1063779616050051

[153] R.C. Lanza, G. Kohse, D. Williams, B.W. Blackburn, R. Sheffield, L. Waters. In Proc. of International topical meeting on nuclear research applications and utilization of accelerators (Vienna, Austria, 2009) p. SM/EN-08.

[154] P.B. Rose, A.S. Erickson, M. Mayer, J. Nattress, I. Jovanovic. Sci. Rep., 6, 24388 (2016). DOI: 10.1038/srep24388

[155] B.E. O’Day, Z.S. Hartwig, R.C. Lanza, A. Danagoulian. Nucl. Instr. And Meth, in Phys. Res. A, 832, 68 (2016). DOI: $10.1016 /$ j.nima.2016.05.117

[156] C.P.J. Barty. In Proc. of the International Symposium, Ed. by H. Takehito (World Scientific Publishing Co. Pte. Ltd., 2015), pp. 85-95. DOI: 10.1142/9789814635455_0011

[157] C. Johnstone, R. Agustsson, S. Boucher, S. Kutsaev, A.Y. Smirnov, R.C. Lanza. In Proc. 22st Int. Conf. on Cyclotrons and their Applications (Cape Town, South Africa, 2019) p. TUPO29. DOI: 10.18429/JACoW-Cyclotrons2019-TUP029

[158] J.-M. Le Goff. Presentation at Technology and innovation workshop European Physical Society (EPS), (Erice, Italy, 2012), available at: https://indico.cern.ch/event/215087/contributions/435158/attachments/342214/477456/Legoff.pdf.

[159] J. Pruet, D.P. McNabb, C.A. Hagmann, F.V. Hartemann, C.P.J. Barty. J. Appl. Phys., 99, 123102-1-11 (2006). DOI: $10.1063 / 1.2202005$

[160] P. Sprangle, A. Ting, E. Esarey, A. Fisher. J. Appl. Phys., 72, 5032 (1992). DOI: 10.1063/1.352031

[161] R. Hajima, T. Hayakawa, N. Kikuzawa, E. Minehara. J. of Nucl. Sci. Tech., 45 (5), 441-451 (2008).

[162] A. Solodko, S. Atieh, N. Catalán Lasheras, A. Grudiev, S. Lebet, W. Wuensch, H. Zha. In Proc. of IPAC2017, (Copenhagen, Denmark, 2017) p. THPAB039. DOI: 10.18429/JACoW-IPAC2017-THPAB039

[163] A. Bacci, D. Alesini, P. Antici, M. Bellaveglia, R. Boni, E. Chiadroni, A. Cianchi, C. Curatolo, G. Di Pirro, A. Esposito, M. Ferrario, A. Gallo, G. Gatti, A. Ghigo, M. Migliorati, A. Mostacci, L. Palumbo, V. Petrillo, R. Pompili, C. Ronsivalle, A.R. Rossi, L. Serafini, B. Spataro, P. Tomassini, C. Vaccarezza. J. Appl. Phys. 113, 194508 (2013). DOI: $10.1063 / 1.4805071$

[164] J.B. Rosenzweig, A. Valloni, D. Alesini, G. Andonian, N. Bernard, L. Faillace, L. Ficcadenti, A. Fukusawa, B. Hidding, M. Migliorati, A. Mostacci, P. Musumeci, B. O'Shea, L. Palumbo, B. Spataro, A. Yakuba, Nucl. Instrum. Methods Phys. Res A., 657 (1), 99 (2011). DOI: 10.1016/j.nima.2011.05.046

[165] S.V. Kutsaev, N.P. Sobenin, A.Yu. Smirnov, D.S. Kamenschikov, M.A. Gusarova, K.I. Nikolskiy, A.A. Zavadtsev, M.V. Lalayan. Nucl. Instrum. Methods Phys. Res. A, 636 (1), 13 (2011). DOI: 10.1016/j.nima.2011.01.047

[166] A. Ovodenko, R. Agustsson, M. Babzien, T. Campese, M. Fedurin, A. Murokh, I. Pogorelsky, M. Polyanskiy, J. Rosenzweig, Y. Sakai, T. Shaftan, C. Swinson. Appl. Phys. Lett. 109, 253504 (2016). DOI: 10.1063/1.4972344

[167] F.E. Carroll, M.H. Mendenhall, R.H. Traeger, C. Brau, J.W. Waters. Am. J Roentgenol., 181 (5), 1197 (2003). DOI: 10.2214/ajr.181.5.1811197

[168] D.F. Sunday, S. List, J.S. Chawla, R.J. Kline. J. Appl. Cryst. 48, 1355 (2015). DOI: 10.1107/S1600576715013369 
[169] D.J. Gibson, S.G. Anderson, F.V. Hartemann, C.W. Siders, A.M. Tremaine, C.P.J. Barty. AIP Conf. Proc., 877, 602 (2006). DOI: $10.1063 / 1.2409190$

[170] A. Bacci, I. Drebot, L. Serafini, V. Torri, V. Petrillo, M. Rossetti Conti, E. Puppin, D. Alesini, M. Bellaveglia, F. Bisesto, B. Buonomo, G. Di Pirro, A. Esposito, F. Iungo, J.J. Beltrano, G. Borgese, G. Di Raddo, A. Gallo, A. Ghigo, L. Pellegrino, A. Stella, C. Vaccarezza, A. Cianchi, R.G. Agostino, R. Barberi, M. Ghedini, F. Martire, C. Pace, G. Dauria, A. Fabris, M. Marazzi. In Proc. of IPAC2016 (Busan, Korea, 2016) p. 1747.

[171] S. Ion. Proc. Math. Phys. Eng. Sci., 473 (2198), 20160815 (2017). DOI: 10.1098/rspa.2016.0815

[172] A.V. Spitsyn, N.P. Bobyr, T.V. Kulevoy, P.A. Fedin, A.I. Semennikov, V.S. Stolbunov. Fusion Eng Des 146 (A), 1313 (2019). DOI: 10.1016/j.fusengdes.2019.02.065

[173] P. Yvon. Structural Materials for Generation IV Nuclear Reactors (Woodhead Publishing, 2017). ISBN: 978-0-08100906-2

[174] Hj. Matzke. Nucl Instrum Meth B., 116 (1-4), 121 (1996). DOI: $10.1016 / 0168-583 X(96) 00021-3$

[175] L.S. Tong. Nucl. Eng. Des., 73(1), 3 (1982). DOI: $10.1016 / 0029-5493(82) 90299-0$

[176] D.G .Cacuci. Handbook of Nuclear Engineering (Springer, Boston, MA, 2010). ISBN: 978-0-387-98130-7

[177] Research reactors for development of materials and fuels for innovative nuclear energy systems (IAEA, Vienna, 2017). ISBN: 978-92-0-100816-9

[178] T.R. Allen, J.T. Busby, R.L. Klueh, S.A. Maloy, M.B. Toloczko. JOM 60 (1), 15 (2018). DOI: $10.1007 / \mathrm{s} 11837-008-0002-6$

[179] S.V. Kutsaev, B. Mustapha, P.N. Ostroumov, J. Nolen, A. Barcikowski, M. Pellin, A. Yacout. Rev. Sci. Instrum., 88, 033302 (2017). DOI: 10.1063/1.4978280

[180] D. Leitner, M.L. Galloway, T.J. Loew, C.M. Lyneis, I. Castro Rodriguez, D.S. Todd. Rev. Sci. Instrum., 79, 02C710 (2008). DOI: 10.1063/1.2816790

[181] P.N. Ostroumov, B. Mustapha, A. Barcikowski, C. Dickerson, A.A. Kolomiets, S.A. Kondrashev, Y. Luo, D. Paskvan, A. Perry, D. Schrage, S.I. Sharamentov, R. Sommer, W. Toter, G. Zinkann. Phys. Rev. Accel. Beams, 15, 110101 (2012). DOI: 10.1103/PhysRevSTAB.15.110101

[182] A.S. Plastun, P.N. Ostroumov. Phys. Rev. Accel. Beams, 21, 030102 (2018). DOI: 10.1103/PhysRevAccelBeams.21.030102

[183] M.A. Green. AIP Conf. Proc., 985, 872 (2008). DOI: $10.1063 / 1.2908683$

[184] P. Hosemann. Rev. Accel. Sci. Technol., 4 (1), 161 (2011). DOI: 10.1142/S1793626811000513

[185] National Research Council. Radiation Source Use and Replacement: Abbreviated Version (The National Academies Press, Washington, DC, USA, 2008). ISBN: 9780-309-11014-3

[186] C.D. Ferguson, T. Kazi, J. Perera. Commercial Radioactive Sources: Surveying the Security Risk (Monterey Institute of International Studies, Washington, DC, USA, 2003). ISBN 1-885350-06-6

[187] G.M. Moore, M.A. Pomper. Permanent Risk Reduction: A Roadmap for Replacing High-Risk Radioactive Sources and Materials (Monterey Institute of International Studies, Washington, DC, USA, 2015). ISBN 978-0-9892361-9-5

[188] R. Talman. Accelerator X-Ray Sources, 1st edition (WileyVCH, Weinheim, 2006). ISBN: 978-3-527405909
[189] International Atomic Energy Agency. Categorization of radioactive sources (IAEA, VIENNA, 2003). ISBN: 92-0105903-5

[190] R. Firestone. Table of Isotopes: 1999 Update, 8th Edition (Wiley-VCH, Weinheim 1999). ISBN: 978-0-471-35633-2

[191] K.E. Ekstrand, W.H. Barnes. Int. J. Radiat. Oncol. Biol. Phys., 18, 249 (1990). DOI: 10.1016/0360-3016(90)90290-Z

[192] C.P. Joshi, S. Dhanesar, J. Darko, A. Kerr, P.B. Vidyasagar, L.J. Schreiner. J. Med. Phys., 34 (3), 137 (2009). DOI: 10.4103/0971-6203.54847

[193] J. W. Wang. High Energy Phys.Nucl.Phys., 30, 11 (2006). Available at: https://www.osti.gov/servlets/purl/897757

[194] T. Yamamoto, T. Natsui, N. Yusa, K. Dobashi, M. Uesaka, T. Higo, S. Fukuda, Mi. Akemoto, M. Yoshida, T. Takatomi, N. Kudoh, E. Tanabe, N. Nakamura, S. Morita, M. Yamamoto. In Proc. of 2007 IEEE International Vacuum Electronics Conference (Kitakyushu, Japan, 2007), p. 1. DOI: 10.1109/IVELEC.2007.4283405

[195] S. Boucher, R. Agustsson, L. Faillace, J. Hartzell, A. Murokh, A. Smirnov, S. Storms, K. Woods. In Proc. of IPAC2012 (New Orleans, LA, USA, 2012) p. THPPR069.

[196] В.В. Парамонов. Автореф. докт. дисс. (Москва, ИЯИ PAH, 2002).

[197] В.И. Каминский. Автореф. докт. дисс. (Москва, НИЯУ МИФИ, 1999).

[198] M.P.J. Gaudreau, J.A. Casey, T.P. Hawkey, J.M. Mulvaney, M.A. Kempkes, P. Ver. In Proc. of the 1999 Particle Accelerator Conf. (New York, USA, 1999) p. 1491.

[199] A.A. Zavadtsev, D.A. Zavadtsev, D.V. Churanov, D.A. Zybin. In Proc. of RuPAC2016 (St. Petersburg, Russia, 2016) p. THPSC027.

[200] D. Delacroix, J.P. Guerre, P. Leblanc, C. Hickman. Radiation Protection Dosimetry - Radionuclide and Radiation Protection Data Handbook (Nuclear Technology Publishing, Kent, 2002). ISBN: 1-870965-87-6

[201] D. Lison. In Handbook on the Toxicology of Metals (3rd Edition) Ed. by G.F. Nordberg (Academic Press, USA, 2007) p. 511. DOI: doi.org/10.1016/B978-012369413-3/50080-X

[202] K.D. Ianakiev, J.M. Goda, T.R. Hill, C.E. Moss, J.J. Ong, M.T. Paffett, R.F. Parker, M.T. Swinhoe. J. Radioanal Nucl. Chem., 282, 657 (2009). DOI: 10.1007/s10967-009-0268-3

[203] K.J. Aaldijk, Th. van der Kaa, V.A. Wichers. International Atomic Energy Agency Report \#IAEA-SM-351/99P (1999). Available at: https://inis.iaea.org/collection/ NCLCollectionStore_Public/30/014/30014819.pdf

[204] A. Lebrun, S.C. Kane, L. Bourva, S. Poirier, N.E. Loghin, D. Langlands. In Proc. of Advancements in Nuclear Instrumentation Measurement Methods and their Applications (ANIMMA) conf. (Marseille, France, 2009) p. 1. DOI: $10.1109 /$ ANIMMA.2009.5503777

[205] D.A. Close, J.C. Pratt, H.F. Atwater. Nucl. Instrum. Meth. Phys. Res. A, 240 (2), 398 (1985). DOI: $10.1016 / 0168-9002(85) 90656-4$

[206] W. Bush, D. Langlands, J. Cooley, N. Tuley. In Proc. of Symposium on international safeguards: Addressing verification challenges (Vienna, Austria, 2006) p. 174.

[207] S. Wichmann, C. Johnson, V. Suarino. J. Nucl. Med. 47 (1), 558 (2006).

[208] H. Griffiths, L. Cohen, S. Watts, E. Mokole, C. Baker, M. Wicks, S. Blunt. Proc. IEEE, 103 (1) 85 (2015). DOI: $10.1109 /$ JPROC.2014.2365517

[209] A.A. Podimsky, N.N. Potrakhov. J. Phys.: Conf. Ser., 808, 012011 (2017). DOI: 10.1088/1742-6596/808/1/012011 
[210] International Atomic Energy Agency. Gamma Irradiators For Radiation Processing (IAEA, Vienna, 2005). ISBN: 83909690-6-8

[211] S. Kumar. J. Nucl. Ene. Sci. Power. Generat. Technol., 5 (1) (2016). DOI: $10.4172 / 2325-9809.1000144$

[212] W. Li, Y. Liua, T. Shu, H. Yang, Y. Fan, C. Yuan, J. Zhang. Phys. Plasmas, 19, 013105 (2012). DOI: 10.1063/1.3677882

[213] F. Lagoutine, J. Legrand, C. Perrot, J.P. Brethon, J. Morel. Int. J. Appl. Radiat. Isotop., 23 (5), 219 (1972). DOI: $10.1016 / 0020-708 X(72) 90058-0$

[214] C. Hellier. Handbook of Nondestructive Evaluation 2nd ed. (McGraw-Hill, New York, 2003), 620 p. ISBN: 9780071777148

[215] M.E. Alden, M. Mohiuddin. Int. J. Radiat. Oncol. Biol. Phys., 28 (4), 945 (1994). DOI: 10.1016/0360-3016(94)90115-5

[216] S. Coll. The Unthinkable (The New Yorker, 2007). Available at: https://www.newyorker.com/magazine/2007/03/ 12/the-unthinkable-2

[217] P. Zou, W. Gai, R. Konecny, X. Sun. Rev. Sci. Instrum., 71, 2301 (2000). DOI: 10.1063/1.1150446

[218] A. Kanareykin, I.L. Sheinman, A.M. Al'tmark. Tech. Phys. Lett., 28, 916 (2002). DOI: 10.1134/1.1526882

[219] M.A. Gusarova, V.I. Kaminsky, L.V. Kravchuk, S.V. Kutsaev, M.V. Lalayan, N.P. Sobenin, S.G. Tarasov. Nucl. Instrum. Methods Phys. Res. A, 599 (1), 100 (2009). DOI: 10.1016/j.nima.2008.09.047

[220] P. Schoessow, S.P. Antipov, C. Jing, A. Kanareykin, S. Zuo, J.G. Power, A. Zholents. In Proc. of PAC2013, (Pasadena, CA USA, 2013) p. MOPAC12.

[221] A.E. Engin, A. Tambawala, M. Swaminathan, S. Bhattacharya, P. Pramanik, K. Yamazaki. In Proc. of 57th Electronic Components and Technology Conference (Reno, NV, 2007) pp. 792-797. DOI: 10.1109/ECTC.2007.373888

[222] A.V. Smirnov, E. Savin. Nucl. Instrum. Methods Phys. Res. A, 820, 48 (2016). DOI: 10.1016/j.nima.2016.03.009

[223] В.Г. Андреев, В.В. Пашковский. ЖТФ, 4 (3), 523 (1970).

[224] V.V. Paramonov. In Proc. of LINAC2012 (Tel-Aviv, Israel, 2012) p. MOPB081.

[225] A.V. Smirnov, S. Boucher, S. Kutsaev, J. Hartzell, E. Savin. Nucl. Instrum. Methods Phys. Res. A, 830, 294 (2016). DOI: 10.1016/j.nima.2016.06.015

[226] D. Satoh, M. Yoshida, N. Hayashizaki. Phys. Rev. Accel. Beams, 19, 011302 (2016). DOI: 10.1103/PhysRevAccelBeams.19.011302

[227] P. Zou, L. Xiao, X. Sun, W. Gai. J. Appl. Phys., 90, 2017 (2001). DOI: $10.1063 / 1.1383578$

[228] R.J. Cameron, C.M. Kudsia, R.R. Mansour. Microwave Filters for Communication Systems: Fundamentals, Design, and Applications (Wiley Telecom, New Jersey, 2018). ISBN: 9781118274347

[229] N. McN Alford, J. Breeze, X. Wang, S.J. Penn, S. Dalla, S.J. Webb, N. Ljepojevic, X. Aupi. J. Eur. Ceram. Soc., 21, 2605 (2001). DOI: 10.1016/S0955-2219(01)00324-7

[230] M. Furuya, A. Ochi. Jpn. J. Appl. Phys., 33, 5482 (1994). DOI: $10.1143 /$ JJAP.33.5482

[231] M.P. Unterweger, D.D. Hoppes, F.J. Schima, J.S. Coursey. Radionuclide Half-Life Measurements Data, (National Institute of Standards and Technology, 2009). Available at: https://www.nist.gov/pml/radionuclide-half-life-measurements/radionuclide-half-life-measurements-data.

[232] Я. ван Коэй. Бюллетень МАГАТЭ, 23 (3), 37 (1981).

[233] J.F. Cameron, M.S. Bourne. Appl. Radiat. Isot., 3 (1), 15 (1958). DOI: 10.1016/0020-708X(58)90051-6
[234] B.Yu. Bogdanovich, K.I. Kozlovskii, A.V. Nesterovich, A.E. Shikanov, A.V. Il'inskii, E.G. Urmanov, At. Energy, 122, 271 (2017). DOI: 10.1007/s10512-017-0266-4

[235] C.C. Fung, K.W. Wong, H. Eren, R. Charlebois. In Proc. of ICNN'95 - International Conference on Neural Networks (Perth, WA, Australia, 1995) v. 1, pp. 526-531. DOI: $10.1109 /$ ICNN.1995.488233

[236] Frontier Technology Corporation. Oil Well \& Borehole Logging, available at: https://www.frontier-cf252.com/welllogging/

[237] B. Steingrímsson. In SDG Short Course III on Geothermal Reservoir Characterization: Well Logging, Well Testing and Chemical Analysis (Santa Tecla, El Salvador, 2018) p. 1.

[238] W.E. Stein. IEEE Trans. Nucl. Sci., 30 (2), 1434 (1983). DOI: $10.1109 /$ TNS.1983.4332553

[239] B. Bogdanovich, V.I. Kaminsky, K. Senyukov, A.V. Nesterovich. In Proc. of the 1997 Part. Acc. Conf. (Vancouver, Canada, 1997) p. 3869. DOI: 10.1109/PAC.1997.753443

[240] J.M. Potter. AIP Conf. Proc., 1525, 178 (2013). DOI: $10.1063 / 1.4802315$

[241] K.E. Nichols, B.E. Carlsten, A. Malyzhenkov. In Proc. of LINAC2016 (East Lansing, MI, USA, 2016) p. MOOP12.

[242] A.V. Smirnov, R.A. Agustsson, S. Boucher, M. Harrison, K. Junge, E. Savin, A.Yu. Smirnov. J. Phys.: Conf. Ser., 941, 012085 (2017). DOI: 10.1088/1742-6596/941/1/012085

[243] A.V. Smirnov. Nucl. Instrum. Methods Phys. Res. A, 868, 39 (2017). DOI: 10.1016/j.nima.2017.06.033

[244] S.K. Esin, L.V. Kravchuk, V.V. Paramonov, G.V. Romanov, A.A. Stepanov. Nucl. Instrum. Meth. B, 68 (1-4), 32 (1992). DOI: 10.1016/0168-583X(92)96045-Z

[245] A.V. Smirnov, E.A. Savin. IOSR-JAP, 10 (2), 65 (2018). DOI: $10.9790 / 4861-1002036577$

[246] World Health Organization. Cancer, available at: https://www.who.int/cancer/resources/keyfacts/en/

[247] R. Atun, D.A. Jaffray, M.B. Barton, F. Bray, M. Baumann, B. Vikram, T.P. Hanna, F.M. Knaul, Y. Lievens, T.Y.M. Lui, M. Milosevic, B. O'Sullivan, D.L Rodin, E. Rosenblatt, J. Van Dyk, M.L. Yap, E. Zubizarreta, M. Gospodarowicz. Lancet Oncol., 16, 1153 (2015). DOI: $10.1016 / \mathrm{S} 1470-2045(15) 00222-3$

[248] S. Gianfaldoni, R. Gianfaldoni, U. Wollina, J. Lotti, G. Tchernev, T. Lotti. Open Access Maced J. Med. Sci., 5 (4), 521 (2017). DOI: 10.3889/oamjms.2017.122

[249] R. Baskar, K.A. Lee, R. Yeo, K.W. Yeoh. Int. J. Med. Sci., 9 (3), 193 (2012). DOI: 10.7150/ijms.3635

[250] A.M. Allen, T. Pawlicki, L. Dong, E. Fourkal, M. Buyyounouski, K. Cengel, J. Plastaras, M.K. Bucci, T.I Yock, L. Bonilla, R. Price, E E. Harris, A.A. Konski. Radiother Oncol., 103, 8 (2012). DOI: $10.1016 /$ j.radonc.2012.02.001

[251] J.E. Turner. Atoms, Radiation, and Radiation Protection, 3rd Edition (Wiley-VCH, N.Y., 2007). ISBN: 978-3527406067

[252] M. Durante, H. Paganetti. „Nuclear physics in particle therapy: a review“, Rep. Prog. Phys., 79, 096702 (2016). DOI: $10.1088 / 0034-4885 / 79 / 9 / 096702$

[253] C. Huff. Hosp Health Netw., 81 (3), 62 (2007). PMID: 17444406

[254] N. Maliwal. Radiotherapy: Technologies and global markets (BCC research, 2015), HLC176B.

[255] E.F. Adebayo, O.A. Uthman, C.S. Wiysonge, E.A. Stern, K.T. Lamont, J.E. Ataguba. BMC Health Serv. Res., 15, 543 (2015). DOI: 10.1186/s12913-015-1179-3 
[256] O. Balogun, D. Rodin, W. Ngwa, S.I. Grover, J. Longo. Semin. Radiat. Oncol., 27 (2), 184 (2017). DOI: 10.1016/j.semradonc.2016.11.011

[257] S. Massoud. In Cancer Control, p. 85-94 (2013). Available at: http://cancercontrol.info/wp-content/uploads/ 2014/08/cc2013_83-96-Samiei-varian-tpage-incld-T-page_ 2012.pdf

[258] P. Dong, P. Lee, D. Ruan, T. Long, E. Romeijn, Y. Yang, D. Low, P. Kupelian, K. Sheng. Int. J. Radiat. Oncol. Biol. Phys., 85, 1360 (2013). DOI: 10.1016/j.jrobp.2012.09.028

[259] UCLA Health. New $4 \pi$ radiation therapy provides treatment option for patients with recurrent glioblastoma, 16v3-09:06-16 (UCLA Health, USA, 2016). Available at: https://www.uclahealth.org/workfiles/clinical_updates/radiation-oncology/ 16v3-09_4piRadiationTherapy_finalHR.pdf

[260] J.C. Rwigema, D. Nguyen, D.E. Heron, A.M. Chen, P. Lee, P. Wang, J.A. Vargo , D.A Low, M.S. Huq, S. Tenn, M.L. Steinberg, P. Kupelian, K. Sheng. Int. J. Radiat. Oncol. Biol. Phys. 91, 401 (2015). DOI: $10.1016 /$ j.ijrobp.2014.09.043

[261] P. Dong, P. Lee, D. Ruan, T. Long, E. Romeijn, D.A. Low, P. Kupelian, J. Abraham, Y. Yang, K. Sheng. Int. J. Radiat. Oncol. Biol. Phys., 86, 407 (2013). DOI: $10.1016 /$ j.jirobp.2013.02.002

[262] Электронный ресурс. Режим доступа: https://blog.peekmed.com/c-arm-x-ray-machines/

[263] Электронный ресурс. Режим доступа: https://cyberknife.com/

[264] V.Y. Yu, A. Tran, D. Nguyen, M. Cao, D. Ruan, D.A. Low, K. Sheng. Med. Phys., 42 (11), 6457 (2015). DOI: $10.1118 / 1.4932631$

[265] K. Woods, M. Harrison, S. Boucher, J. McNevin, S. Kutsaev, L. Faillace, K. Sheng. Med. Phys. 43 (6), 3895 (2016). DOI: $10.1118 / 1.4958253$

[266] N. Linthout, D. Verellen, K. Tournel, T. Reynders, M. Duchateau, G. Storme. Radiother Oncol., 83, 168 (2007). DOI: 10.1016/j.radonc.2007.04.015

[267] P.J. Keall, G.S. Mageras, J.M. Balter, R.S. Emery, K.M. Forster, S.B. Jiang, J.M. Kapatoes, D.A. Low, M.J. Murphy, B.R. Murray, C.R. Ramsey, M.B. Van Herk, S.S. Vedam, J.W. Wong, E. Yorke. Med. Phys., 33 (10), 3874 (2006). DOI: 10.1118/1.2349696

[268] V. Favaudon, L. Caplier, V. Monceau, F. Pouzoulet, M. Sayarath, C. Fouillade, M.F. Poupon, I. Brito, P. Hupé, J. Bourhis, J. Hall, J.J. Fontaine, M.C. Vozenin. Sci. Transl. Med., 16 (6), 245 (2014).

DOI: $10.1126 /$ scitranslmed.3008973

[269] Электронный ресурс. Режим доступа:мwww.varian.com

[270] P. Symonds, G.D.D. Jones. Clin. Oncol. (R. Coll. Radiol.) 31 (7), 405 (2019). DOI: 10.1016/j.clon.2019.05.011

[271] A. Patriarca, C. Fouillade, M. Auger, F. Martin, F. Pouzoulet, C. Nauraye, S. Heinrich, V. Favaudon, S. Meyroneinc, R. Dendale, A. Mazal, P. Poortmans, P. Verrelle, L. De Marzi. Int. J. Radiat. Oncol. Biol. Phys., 102 (3), 619 (2018). DOI: 10.1016/j.jijobp.2018.06.403

[272] Appl. Rad. Oncol. First Flash irradiation delivered in an IBA gantry treatment room (2019). Available at: https:/appliedradiationoncology.com/articles/ first-flashirradiation-delivered-in-an-iba-gantry-treatment-room

[273] M. Krämer, E. Scifoni, C. Schuy, M. Rovituso, W. Tinganelli, A. Maier, R. Kaderka, W. Kraft-Weyrather, S. Brons, T. Tessonnier, K. Parodi, M. Durante. Med. Phys., 43 (4), 1995 (2016). DOI: 10.1118/1.4944593
[274] O. Sokol, E. Scifoni, W. Tinganelli, W. Kraft-Weyrather, J. Wiedemann, A. Maier, D. Boscolo, T. Friedrich, S. Brons, M. Durante, M. Krämer. Phys. Med. Biol., 62 (19), 7798 (2017). DOI: 10.1088/1361-6560/aa88a0

[275] M. Jermann. Hadron therapy patient statistics: Particle therapy cooperative group (2013), Available at:

http://www.ptcog.ch/archive/patient_statistics/PatientstatisticsupdateMar2013.pdf

[276] T. Kamada, H. Tsujii, H. Tsuji, T. Yanagi, J. Mizoe, T. Miyamoto, H. Kato, S. Yamada, S. Morita, K. Yoshikawa, S. Kandatsu, A. Tateishi. J. Clin. Oncol., 20 (22), 4466 (2020). DOI: 10.1200/JCO.2002.10.050

[277] I. Serizawa, K. Kagei, T. Kamada, R. Imai, S. Sugahara, T. Okada, H. Tsuji, H. Ito, H. Tsujii. Int. J. Radiat. Oncol. Biol. Phys., 75 (4), 1105 (2009). DOI: $10.1016 /$ j.ijrobp.2008.12.019

[278] S. Sugahara, T. Kamada, R. Imai, H. Tsuji, N. Kameda, T. Okada, H. Tsujii, S. Tatezaki. Radiother. Oncol., $105(2)$, 226 (2012). DOI: 10.1016/j.radonc.2012.09.010

[279] O. Mohamad, B.J. Sishc, J. Saha, A. Pompos, A. Rahimi, M.D. Story, A.J. Davis, D.W. Nathan Kim. Cancers, 9 (6), 66 (2017). DOI: $10.3390 /$ cancers9060066

[280] C. Schlaff, A. Krauze, A. Belard, J. J. O'Connell, K.A. Camphausen. Radiat. Oncol., 9, 88 (2014). DOI: $10.1186 / 1748-717 X-9-88$

[281] K. Peach, P. Wilson, B. Jones. Br. J. Radiol. 84 (1), S004 (2011). DOI: $10.1259 / \mathrm{bjr} / 16022594$

[282] W.C. Hsi, M.F. Moyers, D. Nichiporov, V. Anferov, M. Wolanski, C.E. Allgower, J.B. Farr, A.E. Mascia, A.N. Schreuder. Med. Phys., 36 (6), 2297 (2009). DOI: $10.1118 / 1.3132422$

[283] G. Kraft. Nucl. Instrum. Methods Phys. Res. A, 454, 1 (2000). DOI: 10.1016/S0168-9002(00)00802-0

[284] U. Amaldi, S. Braccini, P. Puggioni. Rev. Accel. Sci. Technol., 2 (1), 111 (2009). DOI: 10.1142/S179362680900020X

[285] K. Yamanouchi, L. Roso, R. Li, D. Mathur, D. Normand. Progress in Ultrafast Intense Laser Science XII (Springer International Publishing, Switzerland, 2015). ISBN: 978-3319-23657-5

[286] L. Arnaudon, P. Baudrenghien, C. Bertone, Y. Body, J. Broere, O. Brunner, M. Buzio, C. Carli, F. Caspers, J.P. Corso, J. Coupard, A. Dallocchio, N. Dos Santos, R. Garoby, F. Gerigk, L. Hammouti, K. Hanke, M. Jones, I. Kozsar, J. Lettry, J.B. Lallement, A. Lombardi, L.A. LopezHernandez, C. Maglioni, S. Mathot, S. Maury, B. Mikulec, D. Nisbet, C. Noels, M. Paoluzzi, B. Puccio, U. Raich, S. Ramberger, C. Rossi, N. Schwerg, R. Scrivens, G. Vandoni, S. Weisz, J. Vollaire, M. Vretenar, T. Zickler. In Proc. of 2nd International Particle Accelerator Conference IPAC11 (San Sebastian, Spain, 2011) p. TUOAA03.

[287] S. Benedetti, A. Grudiev, A. Latina. Phys. Rev. Accel. Beams, 20, 040101 (2017). DOI: 10.1103/PhysRevAccelBeams.20.040101

[288] C. De Martinis, D. Giove, U. Amaldi, P. Berra, K. Crandall, M. Mauri, M. Weiss, R. Zennaro, E. Rosso, B. Szeless, M. Vretenar, M.R. Masullo, V. Vaccaro, L. Calabretta, A. Rovelli. Nucl. Instrum. Methods Phys. Res. A, 681, 10 (2012). DOI: 10.1016/j.nima.2012.04.017

[289] A.M. Lombardi, V.A. Dimov, M. Garlasché, A. Grudiev, S. Mathot, E. Montesinos, S. Myers, M. Timmins, M. Vretenar. In Proc. IPAC'15 (Richmond, VA, USA, 2015), p. 2408. DOI: 10.18429/JACoW-IPAC2015-WEYB2

[290] M. Vretenar, A. Dallocchio, V.A. Dimov, M. Garlasché, A. Grudiev, A.M. Lombardi, S. Mathot, E. Montesinos, M. Timmins. In Proc. of LINAC2014 (Geneva, Switzerland, 2014) p. THPP040. 
[291] S. Benedetti, A. Degiovanni, A. Grudiev, W. Wuensch, U. Amaldi. In Proc. LINAC'14 (Geneva, Switzerland, 2014) p. THPP061.

[292] A. Vnuchenko, D. Esperante Pereira, B. Gimeno Martinez, S. Benedetti, N. Catalan Lasheras, M. Garlasch, A. Grudiev, G. McMonagle, S. Pitman, I. Syratchev, M. Timmins, R. Wegner, B. Woolley, W. Wuensch, A. Faus Golfe. Phys. Rev. Accel. Beams, 23, 084801 (2020). DOI: 10.1103/PhysRevAccelBeams.23.084801

[293] A. Degiovanni. CERN Yellow Report CERN-2017-004-SP pp. 151-164 (2018). DOI: 10.23730/CYRSP-2017-001.151

[294] U. Masood, T.E. Cowan, W. Enghardt, K.M. Hofmann, L. Karsch, F. Kroll, U. Schramm, J.J. Wilkens, J. Pawelke. Phys. Med. Biol., 62 (13), 5531 (2017). DOI: 10.1088/13616560/aa7124

[295] S. Benedetti. In Proc. 29th Linear Accelerator Conf. LINAC2018 (Beijing, China, 2018) pp. TH1P03. DOI: 10.18429/JACoW-LINAC2018-TH1P03

[296] S. Verdú-Andrés, U. Amaldi, Á. Faus-Golfe. J. Radiat. Res., 54 (1), 1155 (2013). DOI: 10.1093/jrr/rrt053

[297] P.N. Ostroumov, A. Goel, B. Mustapha, A. Nassiri, A.S. Plastun, L. Faillace, S.V. Kutsaev, E.A. Savin. In Proc. NAPAC'16 (Chicago, IL, 2016) p. MOA4CO04.

[298] S.V. Kutsaev, R. Agustsson, S. Boucher, R. Fischer, A. Murokh, B. Mustapha, A. Nassiri, P.N. Ostroumov, A. Plastun, E. Savin, A.Yu. Smirnov. Phys. Rev. Accel. Beams, 20, 120401 (2017). DOI: 10.1103/PhysRevAccelBeams.20.120401

[299] D. Tronc. Nucl. Instrum. Methods Phys. Res A, 327 (2-3), 253 (1993). DOI: 10.1016/0168-9002(93)90689-F

[300] V.V. Paramonov. Phys. Part. Nucl. Lett., 13, 901 (2016). DOI: $10.1134 / \mathrm{S} 1547477116070414$

[301] A.S. Plastun, B. Mustapha, A. Nassiri, P.N. Ostroumov, L. Faillace, S.V. Kutsaev, E.A. Savin. In Proc. of LINAC2016 (East Lansing, MI, USA, 2016), p. THPLR042.

[302] B.M. Oborn, S. Dowdell, P.E. Metcalfe, S. Crozier, R. Mohan, P.J. Keall. Med. Phys., 44 (8), 77 (2017). DOI: $10.1002 / \mathrm{mp} .12371$

[303] M. Nishiuchi, H. Sakaki, T. Hori, P.R. Bolton, K. Ogura, A. Sagisaka, A. Yogo, M. Mori, S. Orimo, A.S. Pirozhkov, I. Daito, H. Kiriyama, H. Okada, S. Kanazawa, S. Kondo, T. Shimomura, M. Tanoue, Y. Nakai, H. Sasao, D. Wakai, H. Daido, K. Kondo, H. Souda, H. Tongu, A. Noda, Y. Iseki, T. Nagafuchi, K. Maeda, K. Hanawa, T. Yoshiyuki, T. Shirai. In Proc. of IPAC'10 (Kyoto, Japan, 2010) p. MOPEA013.

[304] U. Linz, J. Alonso. Phys. Rev. Accel. Beams, 19, 124802 (2016). DOI: 10.1103/PhysRevAccelBeams.19.124802

[305] S. A. Gaillard, T. Kluge, K. A. Flippo, M. Bussmann, B. Gall, T. Lockard, M. Geissel, D.T. Offermann, M. Schollmeier, Y. Sentoku, T.E. Cowan. Phys. Plasmas 18(5), 056710 (2011). DOI: $10.1063 / 1.3575624$

[306] E. d'Humiéres, L. Gremillet. Phys. Plasmas, 12 (6), 062704 (2005). DOI: $10.1063 / 1.1927097$

[307] B.M. Hegelich, D. Jung, B.J. Albright, M. Cheung, B. Dromey, D.C. Gautier, C. Hamilton, S. Letzring, R. Munchhausen, S. Palaniyappan, R. Shah, H.-C. Wu, L. Yin, J.C. Fernández. arXiv:1310.8650 [physics.plasm-ph] (2013).

[308] S.M. Pfotenhauer, O. Jäckel, A. Sachtleben, J. Polz, W. Ziegler, H-P. Schlenvoigt, K-U. Amthor, M.C. Kaluza, K.W.D. Ledingham, R. Sauerbrey, P. Gibbon, A.P.L. Robinson, H. Schwoerer. New J. Phys., 10, 033034 (2008). DOI: 10.1088/1367-2630/10/3/033034
[309] K.W.D. Ledingham, P.R. Bolton, N. Shikazono, C.-M. Charlie. Appl. Sci. 4 (3), 402 (2014). DOI: $10.3390 /$ app4030402

[310] Электронный ресурс. Режим доступа: https://www.varian.com/about-varian/newsroom/press-releases/cyclotron-varian-probeam-compact-proton-therapysystem

[311] Электронный pecypc. Режим доступа: http://wwwelsa.physik.uni-bonn.de/accelerator_list.html

[312] A. Gurevich. Supercond. Sci. Technol., 30, 034004 (2017). DOI: $10.1088 / 1361-6668 / 30 / 3 / 034004$

[313] V. Ganni, P. Knudsen. AIP Conf. Proc., 1573, 1814 (2014). DOI: $10.1063 / 1.4860928$.

[314] R. Kephart, B. Chase, I. Gonin, A. Grassellino, S. Kazakov, T. Khabiboulline, S. Nagaitsev, R. Pasquinelli, S. Posen, O. Pronitchev, A. Romanenko, V. Yakovlev, S. Biedron, S. Milton, N. Sipahi, S. Chattopadhyay, P. Piot. In Proc. of SRF2015 (Whistler, BC, Canada, 2015) p. FRBA03.

[315] T.K. Kroc, C.A. Cooper. Phys. Procedia, 90, 92 (2017). DOI: 10.1016/j.phpro.2017.09.030

[316] T.D. Waite, C.N. Kurucz, W.J. Cooper, D. Brown. In Proc. of Symposium on radiation technology for conservation of the environment (Zakopane, Poland, 1997) p. 187.

[317] A. Saini, N. Aggarwal, A. Sharma, A. Yadav. Biotechnol. Res. Int. 2015, 1 (2015). DOI: 10.1155/2015/157139

[318] M. Ashley Day, F. Beland. US Patent US20190151816A1 (2019).

[319] A.A. Basfar, O.I. Fageeha, N. Kunnummal, S. Al-Ghamdi, A.G. Chmielewski, J. Licki, A. Pawelec, B. Tymiński, Z. Zimek. Fuel, 87 (8-9), 1446 (2008). DOI: $10.1016 /$ j.fuel.2007.09.005

[320] R.D. Kephart. US Patent US9340931B2 (2016).

[321] D.S. Yurov, A.S. Alimov, B.S. Ishkhanov, V.I. Shvedunov. Phys. Rev. Accel. Beams, 20, 044702 (2017). DOI: 10.1103/PhysRevAccelBeams.20.044702

[322] S.B. Felch, M.I. Current, M.C. Taylor. In Proc. of PAC2013 (Pasadena, CA USA, 2013) p. WEYB2.

[323] Y. Maximenko, D.A. Sergatskov, Vy.P. Yakovlev. In Proc. of SRF2011 (Chicago, IL USA, 2011) p. THPO024.

[324] M. Checchin, M. Martinello, A. Romanenko, A. Grassellino, D.A. Sergatskov, S. Posen, O. Melnychuk, J.F. Zasadzinski. Phys. Rev. Applied, 5, 044019 (2016).

DOI: 10.1103/PhysRevApplied.5.044019

[325] Ch. Wang, F.Y. Chang, L.H. Chang, M.H. Chang, J. Chen, L.J. Chen, M.C. Lin, F.T. Chung, Z.K. Liu, C.H. Lo, C.L. Tsai, T.C. Yu, M.S. Yeh. In Proc. of IPAC2017 (Copenhagen, Denmark, 2017) p. MOPVA098.

[326] G. Ciovati, J. Anderson, B. Coriton, J. Guo, F. Hannon, L. Holland, M. LeSher, F. Marhauser, J. Rathke, R. Rimmer, T. Schultheiss, V. Vylet. Phys. Rev. Accel. Beams 21 (9), 091601 (2018).

[327] B. Aune, J. Anderson, B. Coriton, J. Guo, F. Hannon, L. Holland, M. LeSher, F. Marhauser, J. Rathke, R. Rimmer, T. Schultheiss, V. Vylet. Phys. Rev. Accel. Beams, 3(9), 092001 (2000).

DOI: 10.1103/PhysRevAccelBeams.21.091601

[328] W Singer. Supercond. Sci. Technol., 30 (3), 030301 (2017). DOI: $10.1088 / 1361-6668 / 30 / 3 / 033001$

[329] A. Grassellino, A. Romanenko, D. Sergatskov, O. Melnychuk, Y. Trenikhina, A. Crawford, A. Rowe, M. Wong, T. Khabiboulline, F. Barkov. Supercond. Sci. Technol., 26, 102001 (2013). DOI: 10.1088/0953-2048/26/10/102001

[330] D. Gonnella, M. Liepe. In Proc. of LINAC2014 (Geneva, Switzerland, 2014) p. MOPP017. 
[331] A. Romanenko, A. Grassellino, A. C. Crawford, D.A. Sergatskov, O. Melnychukm. Appl. Phys. Lett., 105, 234103 (2014). DOI: 10.1063/1.4903808

[332] S. Posen, M. Liepe. Phys. Rev. Accel. Beams, 17, 112001 (2014). DOI: doi.org/10.1103/PhysRevSTAB.17.112001

[333] S. Holmes, P. Derwent, V. Lebedev, S. Mishra, D. Mitchell, V.P. Yakovlev. In Proc. 6th International Particle Accelerator Conference IPAC2015 (Richmond, VA, USA, 2015), p. 3982. DOI: 10.18429/JACoW-IPAC2015-THPF116

[334] J.N. Galayda. In Proc. IPAC14 (Dresden, Germany, 2014), p. TUIOA04.

[335] G. Eremeev, C.E. Reece, M.J. Kelley, U. Pudasaini, J.R. Tuggle. In Proc. of SRF2015 (Whistler, BC, Canada, 2015) p. TUBA05.

[336] P. Seidel. Applied Superconductivity: Handbook on Devices and Applications (Wiley-VCH, New York, 2015) ISBN: 978-3-527-41209-9

[337] S. Posen, M. Liepe. In Proc. of PAC2013 (Pasadena, CA USA, 2013), p. WEZBA1.

[338] R.E. Laxdal, Y. Ma, P. Harmer, D. Kishi, A. Koveshnikov, N. Muller, A. Vrielink. AIP Conf. Proc. 1573, 1184 (2014). DOI: $10.1063 / 1.4860840$

[339] Электронный ресурс. Режим доступа: https://www.cryomech.com/products/pt420/

[340] S. Kutsaev, R. Agustsson, R. Berry, D. Chao, Z. Conway. J. Phys. Conf. Ser., 1350 (1), 012184 (2019). DOI: 10.1088/1742-6596/1350/1/012184

[341] S. Kutsaev, R. Agustsson, R. Berry, Z.A. Conway, R. Fischer, M.P. Kelly, K. Taletski. IEEE Trans. Appl. Supercond., 30 (8), 1 (2020). DOI: 10.1109/TASC.2020.3002764

[342] A. Nassiri, R.L. Kustom, T. Proslier, T. Tan, X. X. Xi. In Proc. of SRF2013 (Paris, France, 2013) p. TUP086.

[343] R.C. Dhuley, S. Posen, M.I. Geelhoed, O. Prokofiev, J.C.T. Thangaraj, Supercond. Sci. Technol. 33 (6) (2020). DOI: 10.1088/1361-6668/ab82f0

[344] Y. Makhlin, G. Scöhn, A. Shnirman. Nature, 398, 305 (1999). DOI: $10.1038 / 18613$

[345] B. Schumacher. Phys. Rev. A, 51, 2738 (1995). DOI: $10.1103 /$ PhysRevA.51.2738

[346] M. Erhard, M. Krenn, A. Zeilinger. Nat. Rev. Phys., 2, 365 (2020). DOI: 10.1038/s42254-020-0193-5

[347] B.D. Josephson. Phys. Lett., 1 (7), 251 (1962). DOI: $10.1016 / 0031-9163(62) 91369-0$

[348] N. Ofek, A. Petrenko, R. Heeres, P. Reinhold, Z. Leghtas, B. Vlastakis, Y. Liu, L. Frunzio, S.M. Girvin, L. Jiang, M. Mirrahimi, M.H. Devoret, R.J. Schoelkopf. Nature, 536, 441 (2016). DOI: 10.1038/nature18949

[349] H. Paik, D. I. Schuster, Lev S. Bishop, G. Kirchmair, G. Catelani, A.P. Sears, B.R. Johnson, M.J. Reagor, L. Frunzio, L.I. Glazman, S.M. Girvin, M.H. Devoret, R.J. Schoelkopf. Phys. Rev. Lett., 107, 240501 (2011). DOI: doi.org/10.1103/PhysRevLett.107.240501

[350] M. Reagor, W. Pfaff, C. Axline, R.W. Heeres, N. Ofek, K. Sliwa, E. Holland, C. Wang, J. Blumoff, K. Chou, M.J. Hatridge, L. Frunzio, M.H. Devoret, L. Jiang, R.J. Schoelkopf. Phys. Rev. B, 94, 014506 (2016). DOI: 10.1103/PhysRevB.94.014506

[351] A. Romanenko, D.I. Schuster. Phys. Rev. Lett., 119, 264801 (2017). DOI: 10.1103/PhysRevLett.119.264801

[352] S.V. Kutsaev, K. Taletski, R. Agustsson, P. Carriere, A.N. Cleland, Z.A. Conway, É. Dumur, A. Moro, A.Yu. Smirnov. EPJ Quant. Technol., 7, 7 (2020). DOI: $10.1140 /$ epjqt/s40507-020-00082-8
[353] P. Frigola, R. Agustsson, L. Faillace, A. Murokh, G. Ciovati, W. Clemens, P. Dhakal, F. Marhauser, R. Rimmer, J. Spradlin, S. Williams, J. Mireles, P.A. Morton. In Proc. of SRF2015 (Whistler, BC, Canada, 2015), p. THPB042.

[354] A. Romanenko, R. Pilipenko, S. Zorzetti, D. Frolov, M. Awida, S. Belomestnykh, S. Posen, A. Grassellino. Phys. Rev. Appl., 13, 034032 (2020).

DOI: 10.1103/PhysRevApplied.13.034032

[355] J. Palca. Physicists go small: let's put a particle accelerator on a chip, (Wyoming Public Media, Wyoming, 2018), available at: https://www.npr.org/sections/healthshots/2018/07/18/630101228/physicists-go- small-lets-put-aparticle-accelerator-on-a-chip.

[356] Stanford researchers buil a particle accelerator that fits on a chip, miniaturizing a technology that can now find new apliations in research and medicine, Stanford News, (2020), available at: htts://news.stanford.edu/pressreleases/2020/01/02/ accelerator-chiprch-fight-cancer.

[357] K. Wootton, R. Assmann, D. Black, R. Byer, B. Cowan, H.Deng, T.Egenolf, R. England, S. Fan, J. Harris, I. Hartl, P. Hommelhoff, T. Hughes, J. Illmer, R. Ishebeck, F. Kärther, W. Kuropka, Y.J. Lee, A. Li, F. Mayet, J. McNeur, A. Mittelbach, P. Musumeci, U. Niedermayer, M. Qi, L. Rivkin, A. Rühl, N. Sapra, N. Shcönenberger, O. Solgaard, A. Tafel, S. Tantawi, J. Vuckovic. In Proc. of IPAC2017 Conf. (Copenhagen, Denmark, 2017) pp. WEYB1.

[358] D. Gavela, J. Calero, L. García-Tabaés, A. Guirao, D. Obradors-Campos, C. Oliverl, J.M. Pérez Morales, I. Podadera, F. Toral. In Porc. of IPAC'15 (Richmond, VA, USA, 2015), p. 1986.

[359] R.Varela, P. Abramian, J. Calero, P. Calvo, M.Domínguez, A. Estévez, L. García-Tabarés, D. Gavela, P. Gómez, A. Guirao, J.L. Gutiérrez, J.I. Lagares, D. López, L. Martínez, D. Obradors-Campos, C. Oliver, J. Pérez Morales, I. Podadera, F. Toral, C. Vázquez. In Proc. of 5th Inernational Beam Insturmentation Conference IBIC2016 (Barcelona, Spain, 2016), p. MOPG29, DOI: 10.18429/JACoW-IBIC2016-MOPG29.

[360] A smaller, lighter delivery system for protonbeam radiotherapy, MIT News (2015), available at: https://phys.org/news/2015-06-smaller-lighter-deliveryproton-beam- radiotherapy.html

[361] J.V. Minervini, L. Bromberg, P. Michael, A. Radovinsky, D. Winklehner. Harvard Dataverse 1 (2019), DOI: $10.7910 / \mathrm{DVN} / \mathrm{RPON} 1 \mathrm{M}$

[362] D. Satoh, M. Yoshida, N. Hayashizak. Phys. Rev. Accel. Beams, 20, 091302 (2017).

DOI: 10.1103/PhysRevAccelBeams.20.091302

[363] A.V. Smirnov, R. Agustsson, M. Harrison, A. Murokh, A.Yu. Smirnov, S. Boucher, T. Campesee, K.J. Hoyt, E.A. Savin, A.A. Zavadtsev. In Proc of 9th International Particle Accelerator Conference IPAC2018, (Vancouver, BC, Canada, 2018), p. TUZGBF5. DOI: 10.18429/JACoWIPAC2018-TUZGBF5

[364] https://www.uclahealth.org/radonc/4-pi-radiation-therapy

[365] E. Schüler, L. Wang, B.W. Loo, P.G. Maxim. Phys. Med. Biol., 64, 125014 (2019). DOI: 10.1088/1361-6560/ab246f

[366] P.G. Maxim, S.G. Tantawi, B.W. Loo. Radiother Oncol., 139, 28 (2019). DOI: 10.1016/j.radonc.2019.05.005

[367] A miniature accelerator to treat cancer, CERN News (2015), available at: https://phys.org/news/2015-07-miniature-cancer.html 